\title{
Model selection for discrete Markov random fields on graphs
}

\author{
Iara Moreira Frondana
}

PHD THESIS PRESENTED

TO

Institute of Mathematics And Statistics

$\mathrm{OF}$

University OF SÃO PAULO

TO

OBTAIN THE TITLE

$\mathrm{OF}$

DOCTOR IN SCIENCE

Program: Statistics

Advisor: Prof. Florencia Graciela Leonardi

During the development of this work the author received financial support from CAPES and CNPq. This thesis was produced as part of the activities of the Research, Innovation and Dissemination Center for Neuromathematics, funded by FAPESP (grant 2013/07699-0).

São Paulo, May 2016 


\title{
Seleção de modelos para campos aleatórios Markovianos discretos sobre grafos
}

\author{
Iara Moreira Frondana \\ TESE APRESENTADA \\ $\mathrm{AO}$ \\ Instituto DE MATEMÁticA E EstatísticA \\ DA \\ UnIVERSIDADE DE SÃO PAUlO \\ PARA \\ OBTENÇÃO DO TÍTULO \\ $\mathrm{DE}$ \\ DOUTORA EM CIÊNCIAS \\ Programa: Estatística \\ Orientadora: Profa. Dra. Florencia Graciela Leonardi \\ Durante o desenvolvimento deste trabalho a autora recebeu auxílio financeiro da CAPES e CNPq. \\ Esta tese foi produzida como parte das atividades do Centro de Pesquisa, Inovação e Difusão em \\ Neuromatemática, financiado pela FAPESP (processo 2013/07699-0).
}

São Paulo, maio de 2016 
Model selection for discrete Markov random fields on graphs

This is the original version of the thesis elaborated by candidate Iara Moreira Frondana, such as submitted to the Judging Committee. 
Seleção de modelos para campos aleatórios Markovianos discretos sobre grafos

Esta é a versão original da tese elaborada pela candidata Iara Moreira Frondana, tal como submetida à Comissão Julgadora. 


\section{Abstract}

FRONDANA, I. M. Model selection for discrete Markov random fields on graphs. PhD Thesis - Institute of Mathematics and Statistics, University of São Paulo, São Paulo, 2016.

In this thesis we propose to use a penalized maximum conditional likelihood criterion to estimate the graph of a general discrete Markov random field. We prove the almost sure convergence of the estimator of the graph in the case of a finite or countable infinite set of variables. Our method requires minimal assumptions on the probability distribution and contrary to other approaches in the literature, the usual positivity condition is not needed. We present several examples with a finite set of vertices and study the performance of the estimator on simulated data from theses examples. We also introduce an empirical procedure based on $k$-fold cross validation to select the best value of the constant in the estimator's definition and show the application of this method in two real datasets.

Keywords: simple undirected graphs, discrete Markov random fields, model selection, Bayesian information criterion. 


\section{Resumo}

FRONDANA, I. M. Seleção de modelos para campos aleatórios Markovianos discretos sobre grafos. Tese (Doutorado) - Instituto de Matemática e Estatística, Universidade de São Paulo, São Paulo, 2016.

Nesta tese propomos um critério de máxima verossimilhança penalizada para estimar o grafo de dependência condicional de um campo aleatório Markoviano discreto. Provamos a convergência quase certa do estimador do grafo no caso de um conjunto finito ou infinito enumerável de variáveis. Nosso método requer condições mínimas na distribuição de probabilidade e contrariamente a outras abordagens da literatura, a condição usual de positividade não é necessária. Introduzimos alguns exemplos com um conjunto finito de vértices e estudamos o desempenho do estimado em dados simulados desses exemplos. Também propomos um procedimento empírico baseado no método de validação cruzada para selecionar o melhor valor da constante na definição do estimador, e mostramos a aplicação deste procedimento em dois conjuntos de dados reais.

Palavras-chave: grafos simples não-dirigidos, campos aleatórios Markovianos discretos, seleção de modelos, critério da Informação Bayesiano. 


\section{Contents}

List of Figures $\quad$ v

List of Tables $\quad$ vii

1 Indroduction $\quad 1$

2 Discrete Markov random fields on graphs $\quad 3$

2.1 Definitions . . . . . . . . . . . . . . . . . . . . . . . 3

2.2 Basic lemmas . . . . . . . . . . . . . . . . . . . . 4

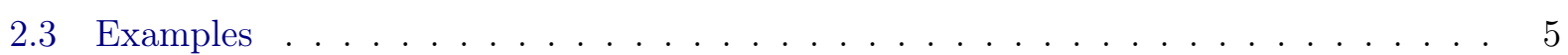

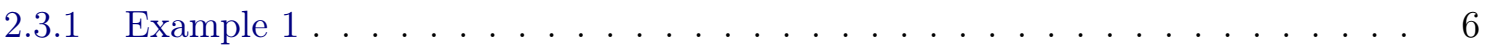

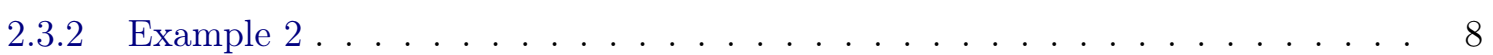

2.3 .3 Example $3 \ldots \ldots \ldots \ldots \ldots \ldots \ldots$

2.3 .4 Example $4 \ldots \ldots \ldots \ldots \ldots \ldots \ldots \ldots$

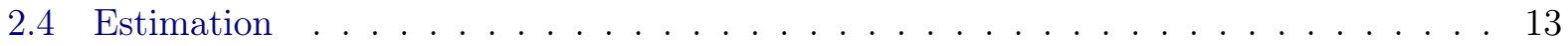

2.5 Model selection . . . . . . . . . . . . . . . . . . . . . . . . . 13

3 Simulations $\quad 20$

3.1 Generating samples . . . . . . . . . . . . . . . . . 20

3.1 .1 Example 1. . . . . . . . . . . . . . . . . . 20

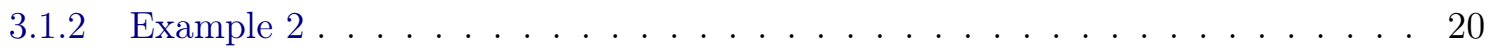

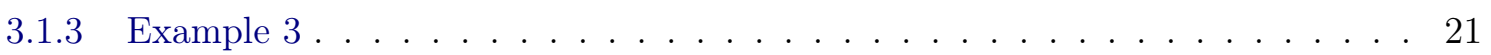

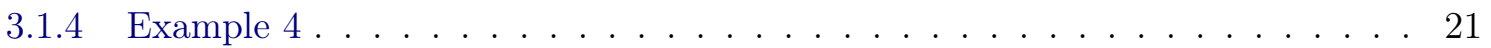

3.2 How to use the estimation program . . . . . . . . . . . . . 21

3.3 Estimator performance . . . . . . . . . . . . . . . . . . . . . 22

3.3.1 Evaluating convergence of the graphs . . . . . . . . . . . . . . . 23

3.3.2 Under and Overestimation errors . . . . . . . . . . . . . . . . . . . . 29

3.3 .3 ROC curves . . . . . . . . . . . . . . . . . . . . . 30

4 Aplications $\quad 35$

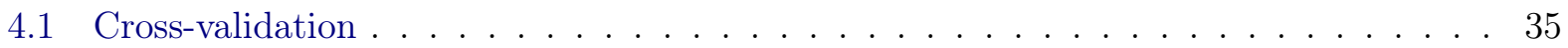

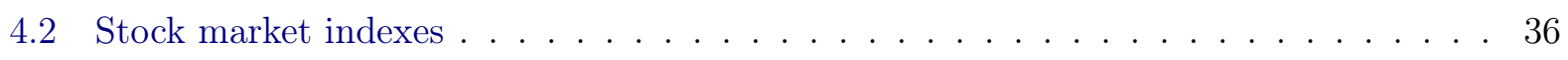

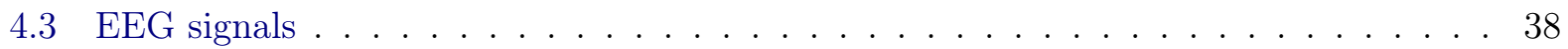


5 Generalization to Markov random fields with countable infinite set of vertices 41 5.1 Basic lemmas . . . . . . . . . . . . . . . . . . . . . . 41

5.2 Model selection when $V$ is countable infinite . . . . . . . . . . . . . . . . 42

6 Conclusion $\quad 51$

$\begin{array}{ll}\text { A Basic probability results } & 53\end{array}$

B R Programs $\quad 55$

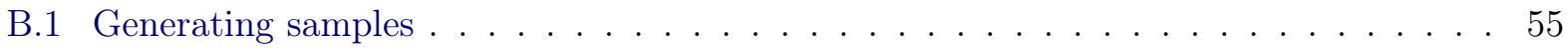

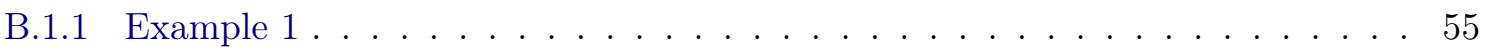

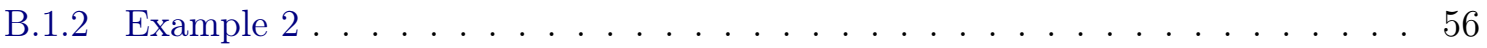

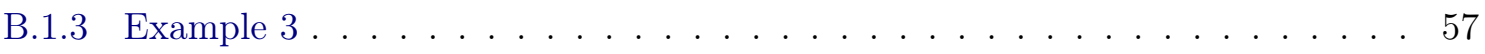

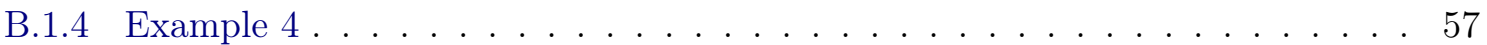

B.2 Programs that measure the estimator performance $\ldots \ldots \ldots \ldots \ldots$

B.3 Log likelihood for cross-validation . . . . . . . . . . . . . . . . . . 60

$\begin{array}{ll}\text { Bibliography } & 62\end{array}$ 


\section{List of Figures}

2.1 Graph of example $1 . \ldots \ldots \ldots \ldots \ldots \ldots \ldots$

2.2 Graph of example $2 \ldots \ldots \ldots \ldots \ldots \ldots \ldots$

2.3 Graph of example $3 . \ldots \ldots \ldots \ldots$

2.4 Graph of example $4 \ldots \ldots \ldots \ldots \ldots \ldots \ldots$

3.1 Graph of example 1: Evolution of the estimated graph for different sample sizes and constant $c$ considering the conservative option. . . . . . . . . . . 23

3.2 Graph of example 1: Evolution of the estimated graph for different sample sizes and constant $c$ considering the non conservative option. . . . . . . . . . 23

3.3 Graph of example 2: Evolution of the estimated graph for different sample sizes and

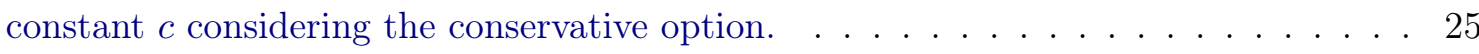

3.4 Graph of example 2: Evolution of the estimated graph for different sample sizes and constant $c$ considering the non conservative option. . . . . . . . . . 25

3.5 Graph of example 3: Evolution of the estimated graph for different sample sizes and

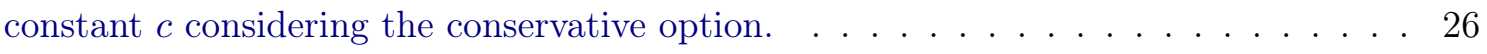

3.6 Graph of example 3: Evolution of the estimated graph for different sample sizes and constant $c$ considering the non conservative option. . . . . . . . . . 26

3.7 Graph of example 4: Evolution of the estimated graph for different sample sizes and

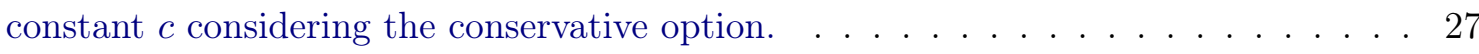

3.8 Graph of example 4: Evolution of the estimated graph for different sample sizes and

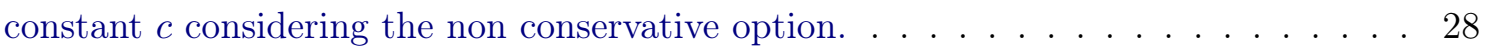

3.9 Graph of example 1: Estimative of the underestimation, overestimantion and total errors for different sample sizes with $c=1 \ldots \ldots \ldots$. . . . . . . . . 29

3.10 Graph of example 2: Estimative of the underestimation, overestimantion and total errors for different sample sizes with $c=1 \ldots \ldots \ldots \ldots$

3.11 Graph of example 3: Estimative of the underestimation, overestimantion and total errors for different sample sizes with $c=1 \ldots \ldots \ldots \ldots \ldots$. . . . . . . . 31

3.12 Graph of example 4: Estimative of the underestimation, overestimantion and total errors for different sample sizes with $c=1 \ldots \ldots \ldots \ldots \ldots \ldots$

3.13 Graph of example 1: ROC curves for $\operatorname{cin}[0 ; 3.5]$ and $n=100 \ldots \ldots \ldots$

3.14 Graph of example 2: ROC curves for $\operatorname{cin}[0 ; 3.5]$ and $n=100 \ldots \ldots \ldots$

3.15 Graph of example 3: ROC curves for $\operatorname{cin}[0 ; 3.5]$ and $n=100 \ldots \ldots \ldots$. . . . . 34

3.16 Graph of example 4: ROC curves for $\operatorname{cin}[0 ; 3.5]$ and $n=100 . \ldots \ldots \ldots$ 
4.1 Stock market indexes from the five countries, from April $18^{\text {th }}$ of 2001 to November

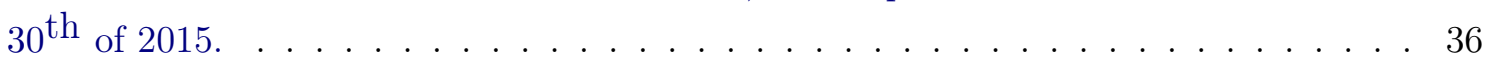

4.2 Stock market indexes: $C V(c)_{10}$, for $c=[0.01,2.5] \ldots \ldots \ldots \ldots \ldots$

4.3 Stock market indexes: graph that represents the relationshio between the countries stock market indexes. . . . . . . . . . . . . . . . . . . . . . . 37

4.4 EEG signals: Position of electrodes on the scalp. . . . . . . . . . . . . . . 38

4.5 EEG signals: The four different graphs used to start the model estimation. . . . . . . 39 


\section{List of Tables}

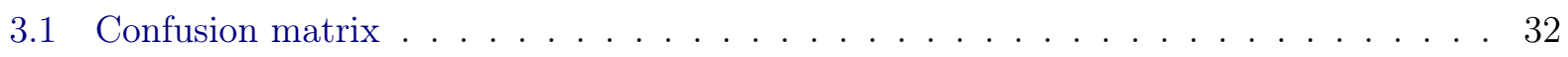




\section{Chapter 1}

\section{Indroduction}

In this thesis we study the problem of model selection for discrete Markov random fields on graphs. These models, also known as graphical models (Lauritzen, 1996) and probabilistic graphical models (Koller and Friedman, 2009), have received much attention from researchers in recent years, especially due to its flexibility to capture conditional dependence between variables. They have been applied to many different problems in different fields such as Biology (Shojaie and Michailidis, 2010) or Social Sciences (Strauss and Ikeda, 1990).

Classically, Markov random fields are defined over a lattice (as for example $\mathbb{Z}^{d}, d \geq 1$ ) and the conditional probability distributions of a variable given the remaining variables are assumed to be translation invariant. The Markov property establishes that these conditional probabilities depend only on a finite set of nearest variables (called Markov neighborhood). In our case, we relax this condition and allow the conditional probability distributions to depend on the specific variable. For this reason the Markov neighborhoods are no longer translation invariant but define a simple and undirected graph. In this thesis we focus the analysis on discrete models, that is the set of random variables takes values on a finite alphabet.

One of the main statistical problems for this type of models is to estimate the underlying graph; that is, the graph determined by the conditional dependence relationships between the variables. For the class of Markov random fields on lattices, some methods based on penalized maximum likelihood criteria like the Bayesian Information Criterion (BIC) of Schwarz (1978) have appeared in the literature (Csiszár and Talata, 2006). For the general class of models defined on graphs, there are some works addressing the problem of model selection but based on other methods. An example of this are the estimators based on the Least Absolute Shrinkage and Selector Operator - LASSO, (Tibshirani, 1996). The LASSO has been applied to estimate the graph for normally distributed variables (Meinshausen and Bühlmann (2006) or for discrete binary alphabets (Ravikumar et al., 2010). Other methods are based on pairwise dependence determination between variables for discrete binary alphabets (Galves et al., 2015) or based on properties of the inverse covariance matrix (Loh and Wainwright, 2013), for general discrete variables.

In general, these works address the problem of consistency of the proposed estimators. In Csiszár and Talata (2006), the consistency of the penalized maximum pseudo-likelihood criterion is proved based on a unique realization of the process. This is only possible due to the translation invariant characteristic of the transition probabilities. In the other works and for general models defined on graphs, the model selection is based on independent realizations of the joint distribution. In the case of the methods based on the LASSO, the proof of the consistency is related to the identification of the non-zero coefficients of a linear regression or logistic (in the binary case) model. One drawback of this approach is the amount of regularity assumptions about the probability distributions, which are practically impossible to be verified in practice. In the case of the approach presented in Galves et al. (2015), the consistency criterion is specific for binary variables and can not be generalized to the general discrete case. The last method is intended for discrete graphical models belonging to the exponential family but is developed only for the special case of multinomial distributions. 
In this thesis we propose to use a penalized maximum conditional likelihood criterion to estimate the graph of a general discrete Markov random field. We prove the almost sure convergence of the estimator of the graph in the case of a finite or countable infinite set of variables. Our method requires minimal assumptions on the probability distribution and contrary to other approaches in the literature, the usual positivity condition is not needed. We present several examples with a finite set of vertices and study the performance of the estimator on simulated data from theses examples. We also introduce an empirical procedure based on $k$-fold cross validation to select the best value of the constant in the estimator's definition and show the application of this method in two real datasets.

This thesis is structured as follows: Chapter 2 presents the definition of the model and the estimator in the case of a finite set of vertices. It also includes some examples and the proof of the consistency of the estimator in this particular case. In Chapter 3 we evaluate the estimator performance through simulations, considering different aspects, such as the sample size, the value of the constant appearing in the definition of the estimator and the choice between a conservative or non conservative approach for the estimation of the full graph. Taking into account the results obtained in the previous chapter, Chapter 4 introduces the empirical method to select the best value of the constant in the estimator's definition and shows the application to the real datasets. In Chapter 5 we revisit the theoretical problem and prove the consistency of a generalized estimator when the set of vertices is countable infinite. Our conclusions are presented in Chapter 6, and Appendices A and B contain some theoretical results needed in this thesis and the $\mathrm{R}$ programs used, respectively. 


\section{Chapter 2}

\section{Discrete Markov random fields on graphs}

This chapter presents the definition of a discrete Markov random field on $A^{V}$ with a graph $G$ along with all the necessary definitions for the correct understanding of the subject. They are also presented some basic lemmas, as well as some examples. Then we show how to estimate the probabilities in a Markov random fiel on $A^{V}$ with graph $G$ when the graph structure is know, and how to estimate the graph through the estimation of the vertices' neighbors using a penalized maximum conditional likelihood method similar to the Bayesian Information Criterion (BIC) of Schwarz (1978).

\subsection{Definitions}

A graph is a pair $G=(V, E)$, where $V$ is the set of vertices and $E$ is the set of edges, $E \subset V \times V$. A graph $G$ is said simple if for all $i \in E,(i, i) \notin E$ and it is said undirected if $(i, j) \in E$ implies $(j, i) \in E$ for all pair $(i, j) \in V \times V$. From this moment on, the word graph is used to denote a simple undirected graph. Given any set $S$, the symbol $|S|$ denotes the cardinality of $S$, where $S$ is a set of vertices or edges.

Let $A$ be a finite set, a random field over $A^{V}$ is a family of random variables indexed by the elements of $V,\left\{X_{v}: v \in V\right\}$, where each $X_{v}$ is a variable with values in $A$. For $\Delta \subseteq V$, a subset of vertices, we write $X_{\Delta}=\left\{X_{i}: i \in \Delta\right\}$, and $a_{\Delta}=\left\{a_{i} \in A: i \in \Delta\right\}$ denotes a configuration on $\Delta$. The joint distribution of the random variables $X_{i}$ (which it is assumed to exist) is denoted by $\mathbb{P}$ :

$$
\mathbb{P}\left(a_{\Delta}\right)=\mathbb{P}\left(X_{\Delta}=a_{\Delta}\right) \text { for } \Delta \subset V \text { finite, } a_{\Delta} \in A^{\Delta} .
$$

And the definition of the conditional probabilities is

$$
\mathbb{P}\left(a_{\Delta} \mid a_{\Phi}\right)=\mathbb{P}\left(X_{\Delta}=a_{\Delta} \mid X_{\Phi}=a_{\Phi}\right) \text { for } a_{\Delta} \in A^{\Delta}, a_{\Phi} \in A^{\Phi}, \mathbb{P}\left(a_{\Phi}\right)>0
$$

for all finite disjoint subsets $\Delta, \Phi \subset V$. From now on, when we write $\mathbb{P}\left(a_{\Delta} \mid a_{\Phi}\right)$ we assume that $\mathbb{P}\left(a_{\Phi}\right)>0$.

A neighborhood $W$ of $v$ is any finite set of vertices with $v \notin W$. And a discrete Markov random field is a random field as described above such that, for all $v \in V$, there is a neighborhood $W$ of $v$, called Markov neighborhood, satisfying

$$
\mathbb{P}\left(a_{v} \mid a_{\Delta}\right)=\mathbb{P}\left(a_{v} \mid a_{W}\right)
$$

for all $\Delta \supset W$ finite, $v \notin \Delta$ and all $a_{v} \in A, a_{\Delta} \in A^{\Delta}$ with $\mathbb{P}\left(a_{\Delta}\right)>0$.

The definition of the Markov neighborhood $W$ is equivalent to request that for all $\Phi$ finite with $\Phi \cap W=\emptyset, X_{\Phi}$ is conditionally independent of $X_{v}$, given $X_{W}$. More formally,

$$
X_{v} \Perp X_{\Phi} \mid X_{W} \text {, for all } \Phi \text { with } \Phi \cap W=\emptyset,
$$


where $\Perp$ is the well known symbol of independence. This corresponds to the property known as local Markov, and it is weaker than the usually assumed global Markov property, see Lauritzen (1996) for details.

A basic fact that we can derive from the definition is that if $W$ is a Markov neighborhood of $v \in V$ then any finite set $\Delta \supset W$ is also a Markov neighborhood of $v$. On the other hand, if $W_{1}$ and $W_{2}$ are Markov neighborhoods of $v$ then it is not always true in general that $W_{1} \cap W_{2}$ is a Markov neighborhood, as shown in the following example.

Example 1. Let $V=\{1,2,3\}$ and consider the vector $\left(X_{1}, X_{2}, X_{3}\right)$ of Bernoulli random variables with $\mathbb{P}\left(X_{i}=0\right)=1 / 2, \mathbb{P}\left(X_{i}=1\right)=1 / 2$, for $i=1,2,3$. Suppose that $X_{1}=X_{2}=X_{3}$ with probability 1. Then it is easy to check that both $\{2\}$ and $\{3\}$ are Markov neighborhoods of node 1 , but the intersection is not a Markov neighborhood (which will imply that $X_{1}$ is independent of $X_{2}$ and $X_{3}$ ).

The intersection property for Markov neighborhoods is desirable in our context to define the smallest Markov neighborhood of a node and to enable the structure estimation problem to be well defined. For that reason we assume the distribution $\mathbb{P}$ satisfies the following:

Markov intersection property: For all $v \in V$ and all $W_{1}$ and $W_{2}$ Markov neighborhoods of $v$, the set $W_{1} \cap W_{2}$ is also a Markov neighborhood of $v$.

This property is guaranteed under the usual positivity condition; namely that all marginal distributions of finite dimension are strictly positive, see Lauritzen (1996). But there are distributions satisfying the Markov intersection property that are not strictly positive. We borrow the following example from Lauritzen (1996), originally presented to show that in general the local and global Markov properties are not equivalent. This example also serves to show that positivity and Markov intersection are not equivalent in general.

Example 2. Let $V=\{1,2,3,4,5\}$ and consider the random vector $\left(X_{1}, X_{2}, X_{3}, X_{4}, X_{5}\right)$ such that: $X_{1}$ and $X_{5}$ are independent, with $\mathbb{P}\left(X_{1}=0\right)=\mathbb{P}\left(X_{1}=1\right)=\mathbb{P}\left(X_{5}=0\right)=\mathbb{P}\left(X_{5}=1\right)=1 / 2$, $X_{2}=X_{1}, X_{4}=X_{5}$ and $X_{3}=X_{2} X_{4}$. Then it can be seen that this distribution satisfies the Markov intersection property for all $v \in V$, but it does not satisfy the positivity condition.

From now on and for the rest of this chapter we will focus on the case where $V$ is a finite set. The case where $V$ is countable infinite will be treated in Chapter 5 . The next section presents the basic lemmas that define the smallest Markov neighborhood of a discrete Markov random field and prove that the graph $G=(V, E)$ cronstructed from these neighborhoods is undirected.

\subsection{Basic lemmas}

Let $\Theta(v)$ be the set of all subsets of $V$ that are Markov neighborhoods of $v \in V$, i.e, $\Theta(v)=$ $\left\{W_{i} \subset V: W_{i}\right.$ is a Markov neighborhood of $\left.v\right\}$. Note that because $V$ is finite, the set $\Theta(v)$ is finite as well. Now, let define ne $(v)$, with $v \in V$, as

$$
\operatorname{ne}(v)=\bigcap_{W_{i} \in \Theta(v)} W_{i} .
$$

Lemma 3. For all $v \in V$ the subset $n e(v) \subset V$ defined by (2.3) is a Markov neighborhood of $v$.

Proof. This lemma is a direct consequence of the Markov intersection property, noticing that ne $(v)$ is a finite intersection of Markov neighborhoods.

By the definition of ne $(v)$ in (2.3) and Lemma 3, ne $(v)$ is the smallest Markov neighborhood of $v \in V$.

The next lemma is an adaptation of Lemma A.2 of Csiszár and Talata (2006). 
Lemma 4. For a Markov random field $\mathbb{P}$, if a neighborhood $W$ satisfies

$$
\mathbb{P}\left(a_{v} \mid a_{W}\right)=\mathbb{P}\left(a_{v} \mid a_{n e(v)}\right) \text { for all } a_{V} \in A^{V}
$$

then $W$ is a Markov neighborhood.

Proof. We have to show that

$$
\mathbb{P}\left(a_{v} \mid a_{V \backslash\{v\}}\right)=\mathbb{P}\left(a_{v} \mid a_{W}\right) \text { for all } a_{V} \in A^{V} .
$$

As ne $(v)$ is a Markov neighborhood, the lemma's condition implies that

$$
\mathbb{P}\left(a_{v} \mid a_{W}\right)=\mathbb{P}\left(a_{v} \mid a_{\mathrm{ne}(v)}\right)=\mathbb{P}\left(a_{v} \mid a_{V \backslash\{v\}}\right) \text { for all } a_{V} \in A^{V} .
$$

So $W$ is a Markov neighborhood.

Given a discrete Markov random field over $A^{V}$, we define the graph $G=(V, E)$ by

$$
(v, w) \in E \text { if and only if } w \in \operatorname{ne}(v) .
$$

The graph $G$ is usually known as graph of interactions of the discrete Markov random field.

Lemma 5. The graph $G$, defined above, is undirected, i.e. if $(v, w) \in E \Rightarrow(w, v) \in E$.

Proof. If $w \notin \operatorname{ne}(v)$ then

$$
\mathbb{P}\left(a_{v} \mid a_{V \backslash\{v\}}\right)=\mathbb{P}\left(a_{v} \mid a_{V \backslash\{v, w\}}\right) \quad \text { for all } a_{V} \in A^{V} .
$$

By the definition of conditional probability and (2.5) we have that

$$
\begin{aligned}
\mathbb{P}\left(a_{v}, a_{w} \mid a_{V \backslash\{v, w\}}\right) & =\mathbb{P}\left(a_{v} \mid a_{w}, a_{V \backslash\{v, w\}}\right) P\left(a_{w} \mid a_{V \backslash\{v, w\}}\right) \\
& =\mathbb{P}\left(a_{v} \mid a_{V \backslash\{v, w\}}\right) P\left(a_{w} \mid a_{V \backslash\{v, w\}}\right) \quad \text { for all } a_{V} \in A^{V} .
\end{aligned}
$$

But, on the other hand,

$$
\mathbb{P}\left(a_{v}, a_{w} \mid a_{V \backslash\{v, w\}}\right)=\mathbb{P}\left(a_{w} \mid a_{v}, a_{V \backslash\{v, w\}}\right) P\left(a_{v} \mid a_{V \backslash\{v, w\}}\right) \quad \text { for all } a_{V} \in A^{V} .
$$

Therefore, by (2.6) and (2.7) we have

$$
\mathbb{P}\left(a_{w} \mid a_{v}, a_{V \backslash\{v, w\}}\right)=P\left(a_{w} \mid a_{V \backslash\{v, w\}}\right) \text { for all } a_{V} \in A^{V} \Longrightarrow v \notin \text { ne }(w) .
$$

The conditional distribution of $X_{v}$ given $X_{\mathrm{ne}(v)}=a_{\mathrm{ne}(v)}$ will be denoted by $\left\{p\left(a \mid a_{\mathrm{ne}(v)}\right)\right\}_{a \in A}$; i.e., for all $a \in A$ we have

$$
p\left(a \mid a_{\mathrm{ne}(v)}\right)=\mathbb{P}\left(X_{v}=a \mid X_{k}=a_{k}, k \in \operatorname{ne}(v)\right) .
$$

Similarly, the joint distribution of $\left(X_{v}, X_{\mathrm{ne}(v)}\right)$ will be denoted by $\left\{p\left(a_{v}, a_{\mathrm{ne}(v)}\right)\right\}_{a_{v} \in A, a_{\mathrm{ne}(v)} \in A^{\mathrm{ne}(v)}}$.

\subsection{Examples}

Here we present four examples of a Markov random field over $A^{V}$ with graph $G$. These examples were constructed with different number of vertices, different conditional dependence structures and different alphabet sizes.

In each one of these examples the joint probabilities were defined as a product of conditional probabilities $p\left(a \mid a_{W}\right)$, where the set $\mathrm{W}$ does not have to be the neighborhood of the vertex. But the 
choice of how the factorization of the joint probability is made defines the conditional dependency structures (i.e., defines the structure of the graph) and allows a simpler way of generating samples from Markov random fields over $A^{V}$ with graph $G$ (Chapter 3 ). For the first example we show how to find the neighborhood of each vertex through the chosen factorization, and this can be replicated to the examples 2, 3 and 4 as well.

Note that the theoretical values of the probabilities are also needed to generate the samples, so for each example these values were chosen without any particular reason, that is, other values could have been chosen.

\subsubsection{Example 1}

This first example has 5 random variables that assume values in $A=\{0,1,2\}$, and its joint probability is given by:

$$
p\left(x_{1}, x_{2}, x_{3}, x_{4}, x_{5}\right)=p\left(x_{2} \mid x_{1}, x_{3}\right) p\left(x_{1} \mid x_{3}\right) p\left(x_{4} \mid x_{3}\right) p\left(x_{5} \mid x_{3}\right) p\left(x_{3}\right)
$$

\section{Graph 1}

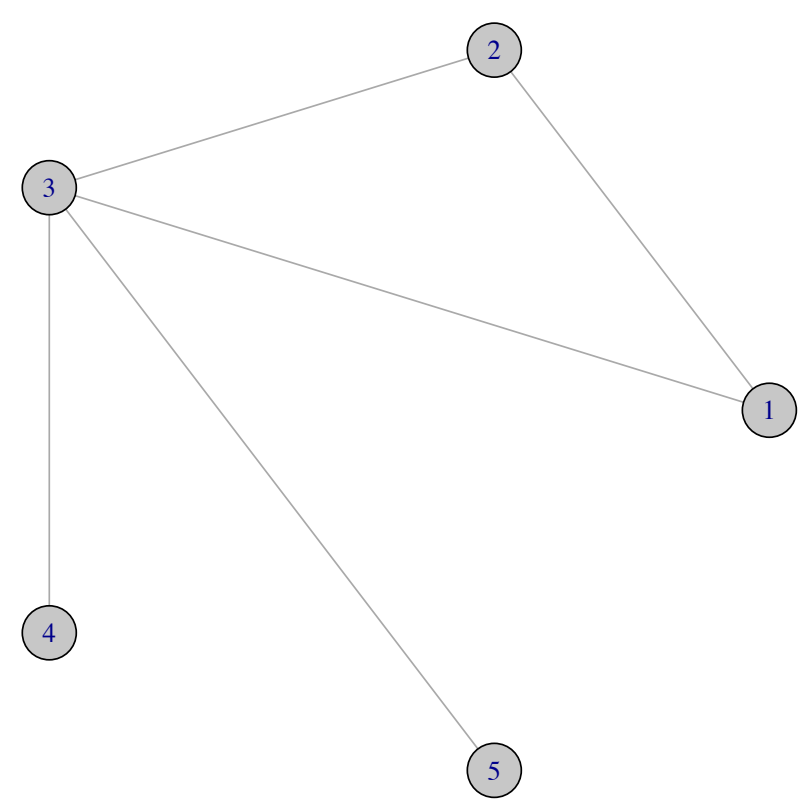

Figure 2.1: Graph of example 1. 
To find the neighborhood of vertex 1 we have from 2.9 :

$$
\begin{aligned}
& p\left(x_{1} \mid x_{2}, x_{3}, x_{4}, x_{5}\right)=\frac{p\left(x_{2} \mid x_{1}, x_{3}\right) p\left(x_{1} \mid x_{3}\right) p\left(x_{4} \mid x_{3}\right) p\left(x_{5} \mid x_{3}\right) p\left(x_{3}\right)}{\sum_{x_{1} \in\{0,1,2\}} p\left(x_{2} \mid x_{1}, x_{3}\right) p\left(x_{1} \mid x_{3}\right) p\left(x_{4} \mid x_{3}\right) p\left(x_{5} \mid x_{3}\right) p\left(x_{3}\right)} \\
= & \frac{p\left(x_{2} \mid x_{1}, x_{3}\right) p\left(x_{1} \mid x_{3}\right) p\left(x_{4} \mid x_{3}\right) p\left(x_{5} \mid x_{3}\right) p\left(x_{3}\right)}{p\left(x_{4} \mid x_{3}\right) p\left(x_{5} \mid x_{3}\right) \sum_{x_{1} \in\{0,1,2\}} p\left(x_{2} \mid x_{1}, x_{3}\right) p\left(x_{1} \mid x_{3}\right) p\left(x_{3}\right)} \\
= & \frac{p\left(x_{2} \mid x_{1}, x_{3}\right) p\left(x_{1} \mid x_{3}\right) p\left(x_{3}\right)}{\sum_{x_{1} \in\{0,1,2\}} p\left(x_{2} \mid x_{1}, x_{3}\right) p\left(x_{1} \mid x_{3}\right) p\left(x_{3}\right)}=\frac{p\left(x_{2} \mid x_{1}, x_{3}\right) p\left(x_{1}, x_{3}\right)}{\sum_{x_{1} \in\{0,1,2\}} p\left(x_{2} \mid x_{1}, x_{3}\right) p\left(x_{1}, x_{3}\right)} \\
= & \frac{p\left(x_{1}, x_{2}, x_{3}\right)}{\sum_{x_{1} \in\{0,1,2\}} p\left(x_{1}, x_{2}, x_{3}\right)}=\frac{p\left(x_{1}, x_{2}, x_{3}\right)}{p\left(x_{2}, x_{3}\right)}=p\left(x_{1} \mid x_{2}, x_{3}\right),
\end{aligned}
$$

so, by 2.10 the neighborhood of vertex 1 is $\{2,3\}$.

The neighborhood of vertex 2 is given by:

$$
\begin{aligned}
& p\left(x_{2} \mid x_{2}, x_{3}, x_{4}, x_{5}\right)=\frac{p\left(x_{2} \mid x_{1}, x_{3}\right) p\left(x_{1} \mid x_{3}\right) p\left(x_{4} \mid x_{3}\right) p\left(x_{5} \mid x_{3}\right) p\left(x_{3}\right)}{\sum_{x_{2} \in\{0,1,2\}} p\left(x_{2} \mid x_{1}, x_{3}\right) p\left(x_{1} \mid x_{3}\right) p\left(x_{4} \mid x_{3}\right) p\left(x_{5} \mid x_{3}\right) p\left(x_{3}\right)} \\
= & \frac{p\left(x_{2} \mid x_{1}, x_{3}\right) p\left(x_{1} \mid x_{3}\right) p\left(x_{4} \mid x_{3}\right) p\left(x_{5} \mid x_{3}\right) p\left(x_{3}\right)}{p\left(x_{1} \mid x_{3}\right) p\left(x_{4} \mid x_{3}\right) p\left(x_{5} \mid x_{3}\right) p\left(x_{3}\right) \sum_{x_{2} \in\{0,1,2\}} p\left(x_{2} \mid x_{1}, x_{3}\right)} \\
= & \frac{p\left(x_{2} \mid x_{1}, x_{3}\right)}{\sum_{x_{2} \in\{0,1,2\}} p\left(x_{2} \mid x_{1}, x_{3}\right)}=\frac{p\left(x_{2} \mid x_{1}, x_{3}\right)}{1}=p\left(x_{2} \mid x_{1}, x_{3}\right)
\end{aligned}
$$

then, by 2.11 , the neighborhood of vertex 2 is $\{1,3\}$.

Now, the neighborhood of vertex 4 is given by:

$$
\begin{aligned}
& p\left(x_{4} \mid x_{2}, x_{3}, x_{4}, x_{5}\right)=\frac{p\left(x_{2} \mid x_{1}, x_{3}\right) p\left(x_{1} \mid x_{3}\right) p\left(x_{4} \mid x_{3}\right) p\left(x_{5} \mid x_{3}\right) p\left(x_{3}\right)}{\sum_{x_{4} \in\{0,1,2\}} p\left(x_{2} \mid x_{1}, x_{3}\right) p\left(x_{1} \mid x_{3}\right) p\left(x_{4} \mid x_{3}\right) p\left(x_{5} \mid x_{3}\right) p\left(x_{3}\right)} \\
= & \frac{p\left(x_{2} \mid x_{1}, x_{3}\right) p\left(x_{1} \mid x_{3}\right) p\left(x_{4} \mid x_{3}\right) p\left(x_{5} \mid x_{3}\right) p\left(x_{3}\right)}{p\left(x_{2} \mid x_{1}, x_{3}\right) p\left(x_{1} \mid x_{3}\right) p\left(x_{5} \mid x_{3}\right) p\left(x_{3}\right) \sum_{x_{4} \in\{0,1,2\}} p\left(x_{4} \mid x_{3}\right)} \\
= & \frac{p\left(x_{4} \mid x_{3}\right)}{\sum_{x_{4} \in\{0,1,2\}} p\left(x_{4} \mid x_{3}\right)}=\frac{p\left(x_{4} \mid x_{3}\right)}{1}=p\left(x_{4} \mid x_{3}\right)
\end{aligned}
$$

then, by 2.12 , the neighborhood of vertex 4 is $\{3\}$. Doing the same for vertex 5 we can find that its neighborhood is $\{3\}$. And, because vertex 3 is in the neighborhood of all other vertices, the neighborhood of vertex 3 is $\{1,2,4,5\}$. Figure 2.1 contains the graph that represents the neighborhoods of the vertices.

The theoretical values chosen for the probabilities in 2.9 are given below:

a) Marginal distribution of $X_{3}$ :

\begin{tabular}{c|ccc}
$x_{3}$ & 0 & 1 & 2 \\
\hline$p\left(x_{3}\right)$ & 0.3 & 0.2 & 0.5
\end{tabular}

b) Conditional probabilities of $X_{i} \mid X_{3}=x_{3}, i=1,4,5$ :

\begin{tabular}{c|ccc}
$x_{1}$ & 0 & 1 & 2 \\
\hline$p\left(x_{1} \mid X_{3}=0\right)$ & 0.2 & 0.4 & 0.4 \\
$p\left(x_{1} \mid X_{3}=1\right)$ & 0.3 & 0.4 & 0.3 \\
$p\left(x_{1} \mid X_{3}=2\right)$ & 0.4 & 0.3 & 0.3 \\
$x_{4}$ & 0 & 1 & 2 \\
\hline$p\left(x_{4} \mid X_{3}=0\right)$ & 0.1 & 0.4 & 0.5 \\
$p\left(x_{4} \mid X_{3}=1\right)$ & 0.2 & 0.7 & 0.1 \\
$p\left(x_{4} \mid X_{3}=2\right)$ & 0.3 & 0.6 & 0.1
\end{tabular}




\begin{tabular}{c|ccc}
$x_{5}$ & 0 & 1 & 2 \\
\hline$p\left(x_{5} \mid X_{3}=0\right)$ & 0.2 & 0.6 & 0.2 \\
$p\left(x_{5} \mid X_{3}=1\right)$ & 0.3 & 0.1 & 0.6 \\
$p\left(x_{5} \mid X_{3}=2\right)$ & 0.4 & 0.3 & 0.3
\end{tabular}

c) Conditional probability of $X_{2} \mid X_{1}=x_{1}, X_{3}=x_{3}$ :

\begin{tabular}{c|ccc}
$x_{2}$ & 0 & 1 & 2 \\
\hline$p\left(x_{2} \mid X_{1}=0, X_{3}=0\right)$ & $1 / 2$ & $1 / 2$ & 0 \\
$p\left(x_{2} \mid X_{1}=1, X_{3}=0\right)$ & $2 / 4$ & $1 / 4$ & $1 / 4$ \\
$p\left(x_{2} \mid X_{1}=2, X_{3}=0\right)$ & $1 / 4$ & $1 / 4$ & $2 / 4$ \\
$p\left(x_{2} \mid X_{1}=0, X_{3}=1\right)$ & $1 / 3$ & 0 & $2 / 3$ \\
$p\left(x_{2} \mid X_{1}=1, X_{3}=1\right)$ & $1 / 4$ & $1 / 4$ & $2 / 4$ \\
$p\left(x_{2} \mid X_{1}=2, X_{3}=1\right)$ & $1 / 3$ & $2 / 3$ & 0 \\
$p\left(x_{2} \mid X_{1}=0, X_{3}=2\right)$ & 0 & $3 / 4$ & $1 / 4$ \\
$p\left(x_{2} \mid X_{1}=1, X_{3}=2\right)$ & $1 / 3$ & $1 / 3$ & $1 / 3$ \\
$p\left(x_{2} \mid X_{1}=2, X_{3}=2\right)$ & $1 / 3$ & $1 / 3$ & $1 / 3$
\end{tabular}

By the theoretical probabilities in (a-c) we can find the model's joint probability distribution for all values in $A^{5}$. For example, the probability of all variables equal to 0 is:

$$
\begin{aligned}
& p\left(X_{1}=0, X_{2}=0, X_{3}=0, X_{4}=0, X_{5}=0\right) \\
= & p\left(X_{2}=0 \mid X_{1}=0, X_{3}=0\right) p\left(X_{1}=0 \mid X_{3}=0\right) p\left(X_{4}=0 \mid X_{3}=0\right) p\left(X_{5}=0 \mid X_{3}=0\right) p\left(X_{3}=0\right) \\
= & 0.2 \times 0.2 \times 0.1 \times 0.2 \times 0.3=0.00024
\end{aligned}
$$

Note that in item c) we have three probabilities equal to zero, this means that in this example we do not have all marginal distributions strictly positive.

\subsubsection{Example 2}

In this model we have 7 random variables that assume values in $A=\{0,1\}$, and its joint probability is given by:

$$
p\left(x_{1}, x_{2}, x_{3}, x_{4}, x_{5}, x_{6}, x_{7}\right)=p\left(x_{1} \mid x_{2}\right) p\left(x_{2} \mid x_{3}\right) p\left(x_{4} \mid x_{3}\right) p\left(x_{3}\right) p\left(x_{5} \mid x_{6}\right) p\left(x_{7} \mid x_{6}\right) p\left(x_{6}\right) .
$$

Through 2.13 we can find the dependence structure of the model represented by the graph of Figure 2.2 .

Below are presented the model's chosen theoretical probabilities:

a) Marginal distributions of $X_{3}$ and $X_{6}$ :

\begin{tabular}{c|cc}
$x_{3}$ & 0 & 1 \\
\hline$p\left(x_{3}\right)$ & 0.4 & 0.6 \\
$x_{6}$ & 0 & 1 \\
\hline$p\left(x_{6}\right)$ & 0.6 & 0.4
\end{tabular}

b) Conditional probabilities of $X_{i} \mid X_{3}=x_{3}, i=2,4$ and $X_{j} \mid X_{6}=x_{6}, j=5,7$ :

\begin{tabular}{c|cc}
$x_{2}$ & 0 & 1 \\
\hline$p\left(x_{2} \mid X_{3}=0\right)$ & 0.4 & 0.6 \\
$p\left(x_{2} \mid X_{3}=1\right)$ & 0.5 & 0.5
\end{tabular}




\section{Graph 2}
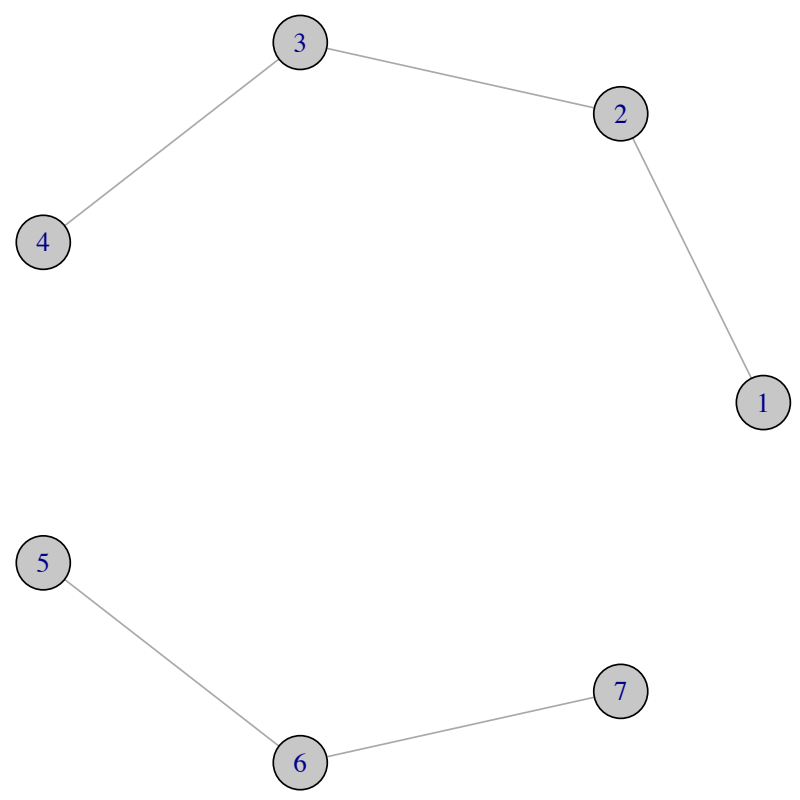

Figure 2.2: Graph of example 2.

$$
\begin{array}{c|cc}
x_{4} & 0 & 1 \\
\hline p\left(x_{4} \mid X_{3}=0\right) & 0.7 & 0.3 \\
p\left(x_{4} \mid X_{3}=1\right) & 0.2 & 0.8 \\
x_{5} & 0 & 1 \\
\hline p\left(x_{5} \mid X_{6}=0\right) & 0.3 & 0.7 \\
p\left(x_{5} \mid X_{6}=1\right) & 0.8 & 0.2 \\
\multicolumn{1}{l}{} \\
x_{7} & 0 & 1 \\
\hline p\left(x_{7} \mid X_{6}=0\right) & 0.5 & 0.5 \\
p\left(x_{7} \mid X_{6}=1\right) & 0.3 & 0.7
\end{array}
$$

c) Conditional probability of $X_{1} \mid X_{2}=x_{2}$ :

\begin{tabular}{c|cc}
$x_{1}$ & 0 & 1 \\
\hline$p\left(x_{1} \mid X_{2}=0\right)$ & 0.3 & 0.7 \\
$p\left(x_{1} \mid X_{2}=1\right)$ & 0.6 & 0.4
\end{tabular}

In this example the probability of all variables be equal to 0 is:

$$
\begin{aligned}
& p\left(X_{1}=0, X_{2}=0, X_{3}=0, X_{4}=0, X_{5}=0, X_{6}=0, X_{7}=0\right) \\
= & p\left(X_{1}=0 \mid X_{2}=0\right) p\left(X_{2}=0 \mid X_{3}=0\right) p\left(X_{4}=0 \mid X_{3}=0\right) p\left(X_{3}=0\right) \\
& p\left(X_{5}=0 \mid X_{6}=0\right) p\left(X_{7}=0 \mid X_{6}=0\right) p\left(X_{6}=0\right) \\
= & 0.3 \times 0.4 \times 0.7 \times 0.4 \times 0.3 \times 0.5 \times 0.6=0.003024
\end{aligned}
$$




\subsubsection{Example 3}

This third model has 12 random variables that assume values in $A=\{0,1,2\}$. And we assume that this model is a homogeneous Markov chain (represented in Figure 2.3), so the model's joint probability can be written as:

$$
p\left(x_{1}, x_{2}, x_{3}, x_{4}, x_{5}, x_{6}, x_{7}, x_{8}, x_{9}, x_{10}, x_{11}, x_{12}\right)=p\left(x_{1}\right) \prod_{i=1}^{11} p\left(x_{i+1} \mid x_{i}\right)
$$

\section{Graph 3}

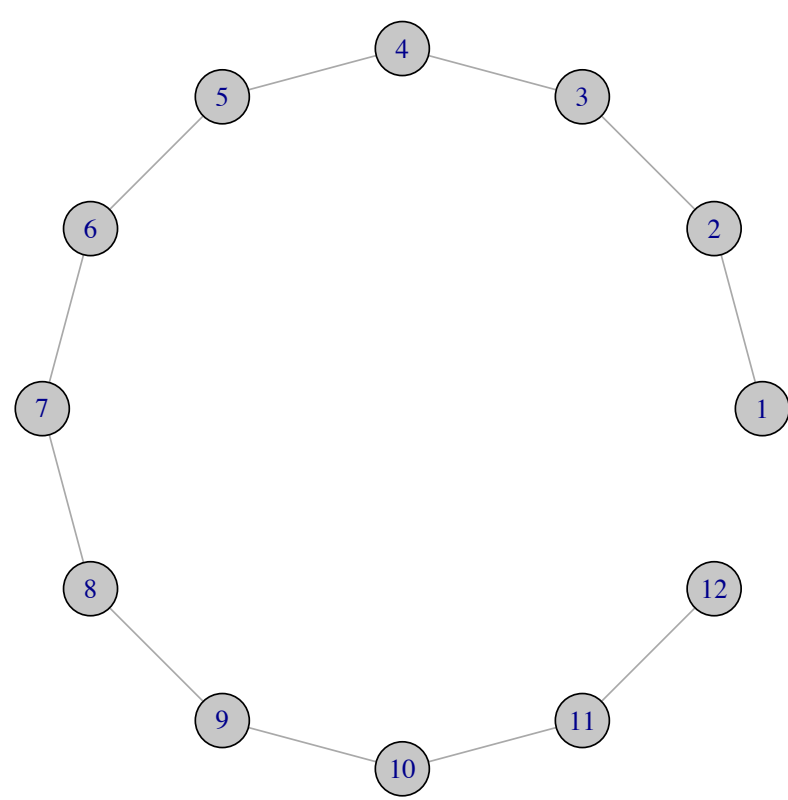

Figure 2.3: Graph of example 3.

And the chosen initial distribution and transition matrix of the Markov chain are:

$$
p\left(x_{1}\right)=\left(\begin{array}{lll}
0.3 & 0.6 & 0.1
\end{array}\right) \quad \text { e } \quad \pi=\left[\begin{array}{lll}
0.2 & 0.5 & 0.3 \\
0.7 & 0.2 & 0.1 \\
0.4 & 0.3 & 0.3
\end{array}\right] .
$$

For this third example the probability of all variables be equal to 0 is:

$$
\begin{aligned}
& p\left(X_{1}=0, X_{2}=0, X_{3}=0, X_{4}=0, X_{5}=0, X_{6}=0, X_{7}=0, X_{8}=0, X_{9}=0,\right. \\
& \left.X_{10}=0, X_{11}=0, X_{12}=0\right) \\
= & p\left(X_{1}=0\right) \prod_{i=1}^{11} p\left(X_{i+1}=0 \mid X_{i}=0\right) \\
= & 0.3 \times 0.2^{11}=6,144 \times 10^{-9}
\end{aligned}
$$




\subsubsection{Example 4}

In this last example we have 10 random variables that assume values in $A=\{0,1,2\}$, and its joint probability is given by

$$
\begin{aligned}
& p\left(x_{1}, x_{2}, x_{3}, x_{4}, x_{5}, x_{6}, x_{7}, x_{8}, x_{9}, x_{10}\right) \\
= & p\left(x_{1} \mid x_{2}\right) p\left(x_{2} \mid x_{4}\right) p\left(x_{3} \mid x_{4}\right) p\left(x_{5} \mid x_{4}\right) p\left(x_{6} \mid x_{4}\right) p\left(x_{4}\right) \\
& p\left(x_{7} \mid x_{10}\right) p\left(x_{8} \mid x_{9}, x_{10}\right) p\left(x_{9} \mid x_{10}\right) p\left(x_{10}\right)
\end{aligned}
$$

The dependence structure can be seen in Figure 2.4, and the theoretical values chosen for the probabilities are given below:

a) Marginal distributions of $X_{4}$ and $X_{10}$ :

\begin{tabular}{c|ccc}
$x_{4}$ & 0 & 1 & 2 \\
\hline$p\left(x_{4}\right)$ & 0.3 & 0.4 & 0.3 \\
$x_{10}$ & 0 & 1 & 2 \\
\hline$p\left(x_{10}\right)$ & 0.4 & 0.1 & 0.5
\end{tabular}

\section{Graph 4}
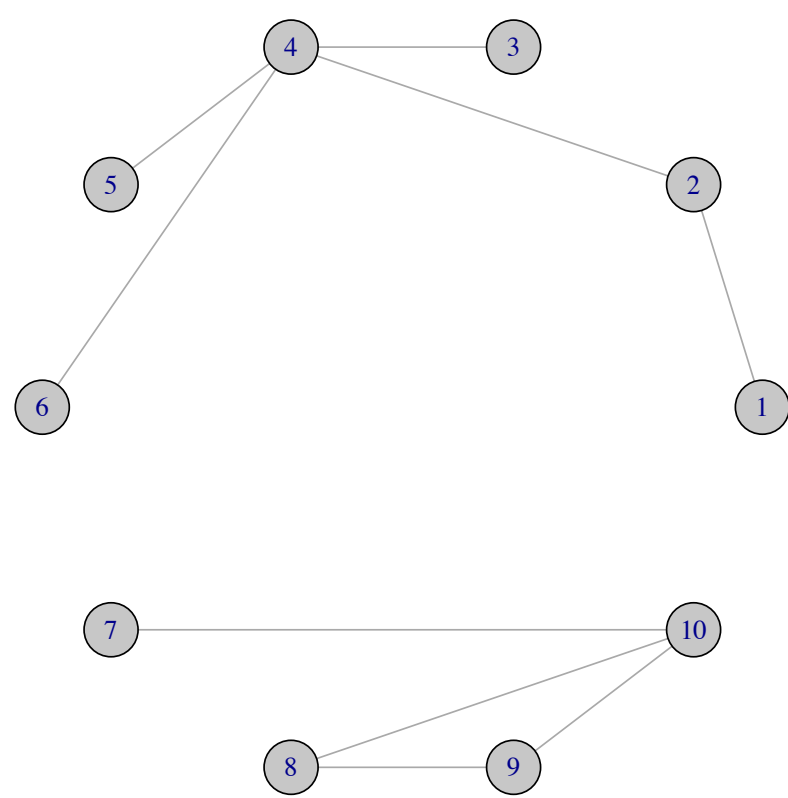

Figure 2.4: Graph of example 4.

b) Conditional probabilities of $X_{i} \mid X_{4}=x_{4}, i=2,3,5,6$ and $X_{j} \mid X_{10}=x_{10}, j=7,9$ :

\begin{tabular}{c|ccc}
$x_{2}$ & 0 & 1 & 2 \\
\hline$p\left(x_{2} \mid X_{4}=0\right)$ & 0.4 & 0.4 & 0.2 \\
$p\left(x_{2} \mid X_{4}=1\right)$ & 0.5 & 0.2 & 0.3 \\
$p\left(x_{2} \mid X_{4}=2\right)$ & 0.5 & 0.3 & 0.2
\end{tabular}




\begin{tabular}{|c|c|c|c|}
\hline$x_{3}$ & 0 & 1 & 2 \\
\hline$p\left(x_{3} \mid X_{4}=0\right)$ & 0.2 & 0.6 & 0.2 \\
\hline$p\left(x_{3} \mid X_{4}=1\right)$ & 0.3 & 0.3 & 0.4 \\
\hline$p\left(x_{3} \mid X_{4}=2\right)$ & 0.4 & 0.2 & 0.4 \\
\hline$x_{5}$ & 0 & 1 & 2 \\
\hline$p\left(x_{5} \mid X_{4}=0\right)$ & 0.7 & 0.2 & 0.1 \\
\hline$p\left(x_{5} \mid X_{4}=1\right)$ & 0.3 & 0.5 & 0.2 \\
\hline$p\left(x_{5} \mid X_{4}=2\right)$ & 0.1 & 0.5 & 0.4 \\
\hline$x_{6}$ & 0 & 1 & 2 \\
\hline$p\left(x_{6} \mid X_{4}=0\right)$ & 0.3 & 0.2 & 0.5 \\
\hline$p\left(x_{6} \mid X_{4}=1\right)$ & 0.6 & 0.2 & 0.2 \\
\hline$p\left(x_{6} \mid X_{4}=2\right)$ & 0.4 & 0.3 & 0.3 \\
\hline$x_{7}$ & 0 & 1 & 2 \\
\hline$p\left(x_{7} \mid X_{10}=0\right)$ & 0.3 & 0.3 & 0.4 \\
\hline$p\left(x_{7} \mid X_{10}=1\right)$ & 0.4 & 0.2 & 0.4 \\
\hline$p\left(x_{7} \mid X_{10}=2\right)$ & 0.5 & 0.3 & 0.2 \\
\hline$x_{9}$ & 0 & 1 & 2 \\
\hline$p\left(x_{9} \mid X_{10}=0\right)$ & 0.4 & 0.4 & 0.2 \\
\hline$p\left(x_{9} \mid X_{10}=1\right)$ & 0.3 & 0.4 & 0.3 \\
\hline$p\left(x_{9} \mid X_{10}=2\right)$ & 0.5 & 0.2 & 0.3 \\
\hline
\end{tabular}

c) Conditional probability of $X_{1} \mid X_{2}=x_{2}$ :

\begin{tabular}{c|ccc}
$x_{1}$ & 0 & 1 & 2 \\
\hline$p\left(x_{1} \mid X_{2}=0\right)$ & 0.2 & 0.3 & 0.5 \\
$p\left(x_{1} \mid X_{2}=1\right)$ & 0.4 & 0.3 & 0.3 \\
$p\left(x_{1} \mid X_{2}=2\right)$ & 0.3 & 0.6 & 0.1
\end{tabular}

d) Conditional probability of $X_{8} \mid X_{9}=x_{9}, X_{10}=x_{10}$ :

\begin{tabular}{c|ccc}
$x_{8}$ & 0 & 1 & 2 \\
\hline$p\left(x_{8} \mid X_{9}=0, X_{10}=0\right)$ & 0.75 & 0 & 0.25 \\
$p\left(x_{8} \mid X_{9}=1, X_{10}=0\right)$ & 0.25 & 0.5 & 0.25 \\
$p\left(x_{8} \mid X_{9}=2, X_{10}=0\right)$ & 0.5 & 0.5 & 0 \\
$p\left(x_{8} \mid X_{9}=0, X_{10}=1\right)$ & 0 & $2 / 3$ & $1 / 3$ \\
$p\left(x_{8} \mid X_{9}=1, X_{10}=1\right)$ & 0.5 & 0.25 & 0.25 \\
$p\left(x_{8} \mid X_{9}=2, X_{10}=1\right)$ & $1 / 3$ & $1 / 3$ & $1 / 3$ \\
$p\left(x_{8} \mid X_{9}=0, X_{10}=2\right)$ & 0.2 & 0.4 & 0.4 \\
$p\left(x_{8} \mid X_{9}=1, X_{10}=2\right)$ & 0.5 & 0 & 0.5 \\
$p\left(x_{8} \mid X_{9}=2, X_{10}=2\right)$ & $1 / 3$ & $1 / 3$ & $1 / 3$
\end{tabular}

For this last example the probability of all variables be equal to 0 is:

$$
\begin{aligned}
& p\left(X_{1}=0, X_{2}=0, X_{3}=0, X_{4}=0, X_{5}=0, X_{6}=0, X_{7}=0, X_{8}=0, X_{9}=0, X_{10}=0\right) \\
= & p\left(X_{1}=0 \mid X_{2}=0\right) p\left(X_{2}=0 \mid X_{4}=0\right) p\left(X_{3}=0 \mid X_{4}=0\right) p\left(X_{5}=0 \mid X_{4}=0\right) \\
& p\left(X_{6}=0 \mid X_{4}=0\right) p\left(X_{4}=0\right) p\left(X_{7}=0 \mid X_{10}=0\right) p\left(X_{8}=0 \mid X_{9}=0, X_{10}=0\right) \\
& p\left(X_{9}=0 \mid X_{10}=0\right) p\left(X_{10}=0\right) \\
= & 0.2 \times 0.4 \times 0.2 \times 0.7 \times 0.3 \times 0.3 \times 0.3 \times 0.75 \times 0.4 \times 0.4=3.6288 \times 10^{-5}
\end{aligned}
$$


As in the first example, here we do not have all marginal distributions strictly positve (look at the probabilities in item d)).

The next section presents how to estimate the probabilities in a Markov random field over $A^{V}$ with graph $G$ when the graph structure is known.

\subsection{Estimation}

Suppose we observe an independent sample with size $n$ of the Markov random field $\left\{X_{v}: v \in V\right\}$. Denote by $x_{v}^{i}$ the value obtained at the vertex $v$ on the $i$-th observation of the sample. For each vertex $v \in V$ we will estimate the neighborhood ne $(v)$ and the conditional probabilities given by (2.8).

Given a vertex $v \in V$ and a set $W \subset V$ not containing $v$, the operator $N\left(a, a_{W}\right)$ will denote the number of occurrences of the event

$$
\left\{X_{v}=a\right\} \cap\left\{X_{W}=a_{W}\right\}
$$

in the sample. That is, for a sample with size $n$

$$
N\left(a, a_{W}\right)=\sum_{i=1}^{n} \mathbf{1}\left\{x_{v}^{i}=a, x_{W}^{i}=a_{W}\right\} .
$$

The maximum likelihood estimator of the conditional distribution (2.8) is given by

$$
\hat{p}\left(a \mid a_{W}\right)=\frac{N\left(a, a_{W}\right)}{N\left(a_{W}\right)}, \quad \text { for } a \in A,
$$

where $N\left(a_{W}\right)=\sum_{a \in A} N\left(a, a_{W}\right)$. If $N\left(a_{W}\right)=0$ we adopt the convention $\hat{p}\left(a \mid a_{W}\right)=1 /|A|$ for all $a \in A$. Therefore, the maximum likelihood of the conditional distribution of $X_{v}$ given $X_{W}$ is

$$
\hat{\mathbb{P}}\left(x_{v}^{(1: n)} \mid x_{W}^{(1: n)}\right)=\prod_{a_{W} \in A^{W}} \prod_{a \in A} \hat{p}\left(a \mid a_{W}\right)^{N\left(a, a_{W}\right)} .
$$

In order to estimate the neighborhood ne $(v)$ of the vertex $v \in V$ we propose a penalized maximum conditional likelihood criterion, similar to the Bayesian Information Criterion (BIC) of Schwarz (1978). In the following section we present the concept of model selection and define the estimators of the neighborhood.

\subsection{Model selection}

Model selection can simply be translated as the task of selecting a statistical model from a set of candidate models given a sample. Since there is the set of candidate models it is necessary to choose the best model among them. However, the meaning of the best can be controversial. A good model selection technique should consider the good fit of the data and the complexity of the model, finding a balance between these two criteria (Wallace and Boulton (1968) and Hastie et al. (2009)). After all, it is obvious that more complex models can incorporate more information coming from the sample, but the added parameters may not represent anything useful to the problem and can increase the variance.

In our case, we look at each vertex of the graph and seek, among a set of possible candidate subsets, the one that has the best fit penalized by the cardinality of this subset. The set $V \backslash\{v\}$ contains all the possible neighbors of $v$, note that we have a total of $\sum_{i=0}^{\mid V \backslash v v\} \mid}\left(\begin{array}{c}|V \backslash\{v\}| \\ i\end{array}\right)=2^{|V \backslash\{v\}|}=$ $2^{|V|-1}$ subsets, that is, we have $2^{|V|-1}$ possible models for the neighborhood of the vertex $v$. Also, note that for the subset $W$ of neighbors and an alphabet $A$, the number of model parameters is 
equal to $(|A|-1)|A|^{|W|}$ and, if the number of neighbors increase in one unit, the number of model parameters increase in

$$
(|A|-1)|A|^{|W|+1}-(|A|-1)|A|^{|W|}=|A|^{|W|+2}-2|A|^{|W|+1}+|A|^{|W|} .
$$

The neighborhood estimator proposed in this thesis uses methods of penalized maximum conditional likelihood similar to the Bayesian Information Criterion (BIC), as already said. In the sequel we define the criterion and prove its consistency.

Definition 6. Given a constant $c>0$, the empirical neighborhood of the vertex $v$ is the set of indices $\widehat{n e}(v)$ given by

$$
\widehat{n e}(v)=\underset{W \subset V \backslash\{v\}}{\arg \max }\left\{\log \hat{\mathbb{P}}\left(x_{v}^{(1: n)} \mid x_{W}^{(1: n)}\right)-c|A|^{|W|} \log _{|A|} n\right\},
$$

where $V \backslash\{v\}$ is the set of all possible neighbors of vertex $v$.

We prove the following consistency result for the neighborhood estimator.

Theorem 7. For any $v \in V$ and any $c>0$, the estimator given by (2.20) satisfies $\widehat{n e}(v)=n e(v)$ eventually almost surely as $n \rightarrow \infty$.

Our main goal in this thesis is to estimate the graph $G$ from a finite sample. To do this we can estimate the neighborhood of each node and reconstruct a graph based on the set of neighborhoods. Based on the neighborhood estimator (2.20), we can construct an estimator of the graph $G$ by defining the set of edges

$$
\widehat{E}^{-}=\{(v, w) \in V \times V: v \in \widehat{\operatorname{ne}}(w) \text { and } w \in \widehat{\operatorname{ne}}(v)\} .
$$

The estimated graph will be the pair $\widehat{G}^{-}=\left(V, \widehat{E}^{-}\right)$. In the same way, if we want to be less conservative we can define

$$
\widehat{E}^{+}=\{(v, w) \in V \times V: v \in \widehat{\operatorname{ne}}(w) \text { or } w \in \widehat{\operatorname{ne}}(v)\}
$$

and the estimated graph will be the pair $\widehat{G}^{+}=\left(V, \widehat{E}^{+}\right)$.

Corollary 8. For all finite $V$ we have $\widehat{G}^{-}=\widehat{G}^{+}=G$ eventually almost surely as $n \rightarrow \infty$.

Before presenting the proof of Theorem 7 and Corollary 8 we prove a proposition that will be used in the proof of those results. And, from now on we simply write $\log n$ for the logarithm in base $|A|$.

Proposition 9. For any $\delta>0$ and any pair $\left(a, a_{W}\right) \in A^{W+1}$ with $W \subset V \backslash\{v\}$ we have

$$
\left|\hat{p}\left(a \mid a_{W}\right)-p\left(a \mid a_{W}\right)\right|<\sqrt{\frac{\delta \log n}{N\left(a_{W}\right)}}
$$

eventually almost surely as $n \rightarrow \infty$.

Proof. Define, for a fixed $\left(a, a_{W}\right) \in A^{W+1}$, the random variables

$$
Y_{i}=\mathbf{1}\left\{x_{v}^{(i)}=a, x_{W}^{(i)}=a_{W}\right\}-p\left(a \mid a_{W}\right) \mathbf{1}\left\{x_{W}^{(i)}=a_{W}\right\}, i=1,2, \ldots, n
$$

and

$$
Z_{n}=\sum_{i=1}^{n} Y_{i}=N\left(a, a_{W}\right)-p\left(a \mid a_{W}\right) N\left(a_{W}\right)
$$


The variables $\left\{Y_{i}: i=1,2, \ldots, n\right\}$ are independent and identically distributed. Note that

$$
\begin{aligned}
& \mathbb{P}\left(Y_{i}=1-p\left(a \mid a_{W}\right)\right)=p\left(a, a_{W}\right), \\
& \mathbb{P}\left(Y_{i}=-p\left(a \mid a_{W}\right)\right)=\sum_{b \neq a} p\left(b, a_{W}\right)=p\left(a_{W}\right)-p\left(a, a_{W}\right), \\
& \mathbb{P}\left(Y_{i}=0\right)=1-p\left(a_{W}\right),
\end{aligned}
$$

where $p\left(a_{W}\right)=\sum_{a^{\prime} \in A} p\left(a^{\prime}, a_{W}\right)$. So, we have that

$$
\begin{aligned}
\mathbb{E}\left(Y_{i}\right)= & \left\{1-p\left(a \mid a_{W}\right)\right\} p\left(a, a_{W}\right)-p\left(a \mid a_{W}\right)\left\{p\left(a_{W}\right)-p\left(a, a_{W}\right)\right\} \\
= & p\left(a, a_{W}\right)-\frac{p\left(a, a_{W}\right)}{p\left(a_{W}\right)} p\left(a, a_{W}\right)-\frac{p\left(a, a_{W}\right)}{p\left(a_{W}\right)} p\left(a_{W}\right) \\
& +\frac{p\left(a, a_{W}\right)}{p\left(a_{W}\right)} p\left(a, a_{W}\right)=0
\end{aligned}
$$

and

$$
\begin{aligned}
\mathbb{E}\left(Y_{i}^{2}\right)= & \left\{1-2 \frac{p\left(a, a_{W}\right)}{p\left(a_{W}\right)}+\frac{p\left(a, a_{W}\right)^{2}}{p\left(a_{W}\right)^{2}}\right\} p\left(a, a_{W}\right) \\
& +\frac{p\left(a, a_{W}\right)^{2}}{p\left(a_{W}\right)^{2}}\left\{p\left(a_{w}\right)-p\left(a, a_{W}\right)\right\} \\
= & \frac{p\left(a, a_{W}\right)}{p\left(a_{W}\right)}\left\{p\left(a_{W}\right)-p\left(a, a_{W}\right)\right\} \\
= & p\left(a \mid a_{W}\right)\left\{1-p\left(a \mid a_{W}\right)\right\} p\left(a_{W}\right) \\
\leq & \frac{1}{4} p\left(a_{W}\right)
\end{aligned}
$$

Now, by the Law of the Iterated Logarithm (Appendix A, 26) we have that for any $\epsilon>0$,

$$
\left|Z_{n}\right|<(1+\epsilon) \frac{p\left(a_{W}\right)}{4} \sqrt{2 n \log \log n}=(1+\epsilon) \frac{1}{4} \sqrt{2 p\left(a_{W}\right)^{2} n \log \log n}
$$

eventually almost surely as $n \rightarrow \infty$. In particular we have

$$
\left|Z_{n}\right|<\sqrt{2 p\left(a_{W}\right)^{2} n \log \log n}
$$

eventually almost surely as $n \rightarrow \infty$. Dividing both sides of the inequality by $N\left(a_{W}\right)$ we obtain that

$$
\left|\hat{p}\left(a \mid a_{W}\right)-p\left(a \mid a_{W}\right)\right|<\sqrt{\frac{2 p\left(a_{W}\right)^{2} n \log \log n}{N\left(a_{W}\right)^{2}}}
$$

eventually almost surely as $n \rightarrow \infty$. By the Strong Law of Large Numbers (Appendix A, 27) $n / N\left(a_{W}\right) \rightarrow 1 / p\left(a_{W}\right)$ almost surely, therefore we have

$$
\left|\hat{p}\left(a \mid x_{W}\right)-p\left(a \mid a_{W}\right)\right|<\sqrt{\frac{2 p\left(a_{W}\right) \log \log n}{N\left(a_{W}\right)}}
$$

eventually almost surely as $n \rightarrow \infty$. Now, for any $\delta>0$ we have

$$
2 p\left(a_{W}\right) \log \log n<\delta \log n
$$


eventually as $n \rightarrow \infty$. Ergo

$$
\left|\hat{p}\left(a \mid a_{W}\right)-p\left(a \mid a_{W}\right)\right|<\sqrt{\frac{\delta \log n}{N\left(a_{W}\right)}} .
$$

Now it is presented the proof of Theorem 7 .

Proof of Theorem \%. For $v \in V$ and $W \subset V \backslash\{v\}$ denote by

$$
\operatorname{PML}\left(x_{v}^{(1: n)} \mid x_{W}^{(1: n)}\right)=\log \hat{\mathbb{P}}\left(x_{v}^{(1: n)} \mid x_{W}^{(1: n)}\right)-c|A|^{|W|} \log n
$$

where

$$
\hat{\mathbb{P}}\left(x_{v}^{(1: n)} \mid x_{W}^{(1: n)}\right)=\prod_{x_{W} \in A^{|W|}} \prod_{a \in A} \hat{p}\left(a \mid a_{W}\right)^{N\left(a, a_{W}\right)} .
$$

We will show that for all $W \subset V \backslash\{v\}, W \neq \operatorname{ne}(v)$

$$
\operatorname{PML}\left(x_{v}^{(1: n)} \mid x_{W}^{(1: n)}\right)<\operatorname{PML}\left(x_{v}^{(1: n)} \mid x_{\mathrm{ne}(v)}^{(1: n)}\right)
$$

eventually almost surely as $n \rightarrow \infty$. As the set of all such $W$ is finite this implies the statement of the theorem. Then let $v \in V$ and let $W \subset V \backslash\{v\}$, with $W \neq$ ne $(v)$. We have the following possibilities

(a) $\operatorname{ne}(v) \subset W$;

(b) $W \subset \operatorname{ne}(v)$;

(c) neither ne $(v) \subset W$ nor $W \subset \operatorname{ne}(v)$.

By ease of exposition we will divide the proof in these three cases.

(a) We have to prove that for all $W \supset \operatorname{ne}(v)$

$$
\operatorname{PML}\left(x_{v}^{(1: n)} \mid x_{W}^{(1: n)}\right)<\operatorname{PML}\left(x_{v}^{(1: n)} \mid x_{\mathrm{ne}(v)}^{(1: n)}\right)
$$

eventually almost surely $n \rightarrow \infty$.

Observe that

$$
\begin{aligned}
& \operatorname{PML}\left(x_{v}^{(1: n)} \mid x_{\mathrm{ne}(v)}^{(1: n)}\right)-\operatorname{PML}\left(x_{v}^{(1: n)} \mid x_{W}^{(1: n)}\right)= \\
& c\left(|A|^{|W|}-|A|^{|\operatorname{ne}(v)|}\right) \log n-\sum_{\left(a, a_{W}\right) \in A^{W+1}} N\left(a, a_{W}\right) \log \frac{\hat{p}\left(a \mid a_{W}\right)}{\hat{p}\left(a \mid a_{\mathrm{ne}(v)}\right)} .
\end{aligned}
$$

As these empirical probabilities are the maximum likelihood estimators, ne $(v) \subset W$ and keeping in mind that ne $(v)$ by definition is the smallest Markov neighborhood of $v$, we have that

$$
\begin{aligned}
\sum_{\left(a, a_{W}\right) \in A^{W+1}} N\left(a, a_{W}\right) \log \hat{p}\left(a \mid a_{\mathrm{ne}(v)}\right) & \geq \sum_{\left(a, a_{W}\right) \in A^{W+1}} N\left(a, a_{W}\right) \log p\left(a \mid a_{\mathrm{ne}(v)}\right) \\
& =\sum_{\left(a, a_{W}\right) \in A^{W+1}} N\left(a, a_{W}\right) \log p\left(a \mid a_{W}\right) .
\end{aligned}
$$

Therefore, (2.23) can be lower-bounded by

$$
c\left(1-\frac{1}{|A|}\right)|A|^{|W|} \log n-\sum_{\left(a, a_{W}\right) \in A^{W+1}} N\left(a, a_{W}\right) \log \frac{\hat{p}\left(a \mid a_{W}\right)}{p\left(a \mid a_{W}\right)} .
$$


Now, observe that

$$
\sum_{\left(a, a_{W}\right) \in A^{W+1}} N\left(a, a_{W}\right) \log \frac{\hat{p}\left(a \mid a_{W}\right)}{p\left(a \mid a_{W}\right)}=\sum_{a_{W} \in A^{W}} N\left(a_{W}\right) D\left(\hat{p}\left(\cdot \mid a_{W}\right) ; p\left(\cdot \mid a_{W}\right)\right),
$$

where $D$ denotes the Küllback-Leibler divergence (see Definition 22 in Appendix A). Therefore we have, by Lemma 24 (in Appendix A) and Proposition 9, that for any $\delta>0$

$$
\begin{aligned}
\sum_{a_{W} \in A^{W}} N\left(a_{W}\right) & D\left(\hat{p}\left(\cdot \mid a_{W}\right) ; p\left(\cdot \mid a_{W}\right)\right) \\
& \leq \sum_{a_{W} \in A^{W}} N\left(a_{W}\right) \sum_{a \in A} \frac{\left[\hat{p}\left(a \mid a_{W}\right)-p\left(a \mid a_{W}\right)\right]^{2}}{p\left(a \mid a_{W}\right)} \\
& \leq \sum_{a_{W} \in A^{W}} N\left(a_{W}\right) \sum_{a \in A} \frac{\delta \log n}{N\left(a_{W}\right) p\left(a \mid a_{W}\right)} \\
& \leq \frac{\delta|A|^{|W|+1} \log n}{p_{\min }}
\end{aligned}
$$

eventually almost surely as $n \rightarrow \infty$, where

$$
p_{\min }=\min \left\{p\left(a \mid a_{W}\right): p\left(a \mid a_{W}\right)>0, a \in A, a_{W} \in A^{W}\right\} .
$$

Then, if we take $c>\frac{\delta|A|^{2}}{p_{\min }(|A|-1)}$, we have that eventually almost surely as $n \rightarrow \infty$

$$
\operatorname{PML}\left(x_{v}^{(1: n)} \mid x_{\mathrm{ne}(v)}^{(1: n)}\right)>\operatorname{PML}\left(x_{v}^{(1: n)} \mid x_{W}^{(1: n)}\right) .
$$

This completes the proof of part (a).

(b) We have to prove that for all $W \subset \operatorname{ne}(v)$

$$
\operatorname{PML}\left(x_{v}^{(1: n)} \mid x_{\mathrm{ne}(v)}^{(1: n)}\right)>\operatorname{PML}\left(x_{v}^{(1: n)} \mid x_{W}^{(1: n)}\right)
$$

eventually almost surely as $n \rightarrow \infty$.

In this case we have that

$$
\begin{aligned}
\operatorname{PML}\left(x_{v}^{(1: n)} \mid x_{\mathrm{ne}(v)}^{(1: n)}\right)-\operatorname{PML}\left(x_{v}^{(1: n)} \mid x_{W}^{(1: n)}\right)= & \sum_{\left(a, a_{\mathrm{ne}(v)}\right) \in A^{\mathrm{ne}(v)+1}} N\left(a, a_{\mathrm{ne}(v)}\right) \log \frac{\hat{p}\left(a \mid a_{\mathrm{ne}(v)}\right)}{\hat{p}\left(a \mid a_{W}\right)}-c\left(|A|^{|\mathrm{ne}(v)|}-|A|^{|W|}\right) \log n \\
= & n\left[\sum_{\left(a, a_{\mathrm{ne}(v)}\right) \in A^{\mathrm{ne}(v)+1}} \frac{N\left(a, a_{\mathrm{ne}(v)}\right)}{n} \log \frac{\hat{p}\left(a \mid a_{\mathrm{ne}(v)}\right)}{\hat{p}\left(a \mid a_{W}\right)}-c\left(|A|^{|\operatorname{ne}(v)|}-|A|^{|W|}\right) \frac{\log n}{n}\right] .
\end{aligned}
$$

By the Strong Law of Large Numbers (Appendix A, Theorem 26) we have that

$$
\begin{aligned}
& \sum_{\left(a, a_{\mathrm{ne}(v)}\right) \in A^{\mathrm{ne}(v)+1}} \frac{N\left(a, a_{\mathrm{ne}(v)}\right)}{n} \log \frac{\hat{p}\left(a \mid a_{\mathrm{ne}(v)}\right)}{\hat{p}\left(a \mid a_{W}\right)} \\
& \longrightarrow \sum_{\left(a, a_{\mathrm{ne}(v)}\right) \in A^{\mathrm{ne}(v)+1}} p\left(a, a_{\mathrm{ne}(v)}\right) \log \frac{p\left(a \mid a_{\mathrm{ne}(v)}\right)}{p\left(a \mid a_{W}\right)}
\end{aligned}
$$


almost surely as $n \rightarrow \infty$. On the other hand,

$$
c\left(|A|^{|\operatorname{ne}(v)|}-|A|^{|W|}\right) \frac{\log n}{n} \longrightarrow 0
$$

when $n \rightarrow \infty$. Now, note that we can rewrite the right-hand side of (2.24) by

$$
\begin{aligned}
\sum_{\left(a, a_{\mathrm{ne}(v)}\right) \in A^{\mathrm{ne}(v)+1}} p\left(a, a_{\mathrm{ne}(v)}\right) \log \frac{p\left(a \mid a_{\mathrm{ne}(v)}\right)}{p\left(a \mid a_{W}\right)} & \\
= & \sum_{x_{\mathrm{ne}(v)} \in A^{\mathrm{ne}(v)}} p\left(a_{\mathrm{ne}(v)}\right) \sum_{a \in A} p\left(a \mid a_{\mathrm{ne}(v)}\right) \log \frac{p\left(a \mid a_{\mathrm{ne}(v)}\right)}{p\left(a \mid a_{W}\right)} \\
= & \sum_{a_{\mathrm{ne}(v)} \in A^{\mathrm{ne}(v)}} p\left(a_{\mathrm{ne}(v)}\right) D\left(p\left(. \mid a_{\mathrm{ne}(v)}\right) ; p\left(. \mid a_{W}\right)\right) .
\end{aligned}
$$

By Lemma 23 (in Appendix A) and the minimality of ne $(v)$ (see (2.3) and Lemmas 3 and 4 ) we must have $D\left(p\left(. \mid a_{\mathrm{ne}(v)}\right) ; p\left(. \mid a_{W}\right)\right)>0$ for at least one $a_{\mathrm{ne}(v)}$, so

$$
\sum_{a_{\mathrm{ne}(v)} \in A^{\mathrm{ne}(v)}} p\left(a_{\mathrm{ne}(v)}\right) D\left(p\left(. \mid x_{\mathrm{ne}(v)}\right) ; p\left(. \mid a_{W}\right)\right)>0 .
$$

Therefore

$$
\operatorname{PML}\left(x_{v}^{(1: n)} \mid x_{\mathrm{ne}(v)}^{(1: n)}\right)>\operatorname{PML}\left(x_{v}^{(1: n)} \mid x_{W}^{(1: n)}\right)
$$

eventually almost surely as $n \rightarrow \infty$, completing the proof of case (b).

(c) In this case, let $W$ such that neither $W \subset \operatorname{ne}(v)$ nor ne $(v) \subset W$, and consider $W^{\prime}=W \cup \operatorname{ne}(v)$. We will prove that

$$
\operatorname{PML}\left(x_{v}^{(1: n)} \mid x_{W}^{(1: n)}\right)<\operatorname{PML}\left(x_{v}^{(1: n)} \mid x_{W^{\prime}}^{(1: n)}\right)<\operatorname{PML}\left(x_{v}^{(1: n)} \mid x_{\mathrm{ne}(v)}^{(1: n)}\right)
$$

eventually almost surely as $n \rightarrow \infty$.

As $W^{\prime} \supset$ ne $(v)$, the second inequality is valid by case (a) proved above, so we just have to prove the first inequality. Note that we have

$$
\begin{aligned}
& \operatorname{PML}\left(x_{v}^{(1: n)} \mid x_{W^{\prime}}^{(1: n)}\right)-\operatorname{PML}\left(x_{v}^{(1: n)} \mid x_{W}^{(1: n)}\right)= \\
& \sum_{\left(a, a_{W^{\prime}}\right) \in A^{W^{\prime}+1}} N\left(a, a_{W^{\prime}}\right) \log \frac{\hat{p}\left(a \mid a_{W^{\prime}}\right)}{\hat{p}\left(a \mid a_{W}\right)}-c\left(|A|^{\left|W^{\prime}\right|}-|A|^{|W|}\right) \log n \\
& =n\left[\sum_{\left(a, a_{W^{\prime}}\right) \in A^{W^{\prime}+1}} \frac{N\left(a, a_{W^{\prime}}\right)}{n} \log \frac{\hat{p}\left(a \mid a_{W^{\prime}}\right)}{\hat{p}\left(a \mid a_{W}\right)}-c\left(|A|^{\left|W^{\prime}\right|}-|A|^{|W|}\right) \frac{\log n}{n}\right] .
\end{aligned}
$$

By the Strong Law of Large Numbers (Appendix A, Theorem 26) we have that the first term in the brackets

$$
\begin{aligned}
\sum_{\left(a, a_{W^{\prime}}\right) \in A^{W^{\prime}+1}} \frac{N\left(a, a_{W^{\prime}}\right)}{n} & \log \frac{\hat{p}\left(a \mid a_{W^{\prime}}\right)}{\hat{p}\left(a \mid a_{W}\right)} \\
& \longrightarrow \sum_{\left(a, a_{W^{\prime}}\right) \in A^{W^{\prime}+1}} p\left(a, a_{W^{\prime}}\right) \log \frac{p\left(a \mid a_{W^{\prime}}\right)}{p\left(a \mid a_{W}\right)}
\end{aligned}
$$

almost surely as $n \rightarrow \infty$. On the other hand the second term

$$
c\left(|A|^{\left|W^{\prime}\right|}-|A|^{|W|}\right) \frac{\log n}{n} \longrightarrow 0
$$


when $n \rightarrow \infty$. Now, note that

$$
\begin{array}{rl}
\sum_{\left(a, a_{W^{\prime}}\right) \in A^{W^{\prime}+1}} & p\left(a, a_{W^{\prime}}\right) \log \frac{p\left(a \mid a_{W^{\prime}}\right)}{p\left(a \mid a_{W}\right)} \\
= & \sum_{a_{W^{\prime}} \in A^{W^{\prime}}} p\left(a_{W^{\prime}}\right) \sum_{a \in A} p\left(a \mid a_{W^{\prime}}\right) \log \frac{p\left(a \mid a_{W^{\prime}}\right)}{p\left(a \mid a_{W}\right)}= \\
= & \sum_{a_{W^{\prime}} \in A^{W^{\prime}}} p\left(a_{W^{\prime}}\right) D\left(p\left(\cdot \mid a_{W^{\prime}}\right) ; p\left(\cdot \mid a_{W}\right)\right) .
\end{array}
$$

By Lemma 23 (in Appendix A), $D\left(p\left(. \mid a_{W^{\prime}}\right) ; p\left(. \mid a_{W}\right)\right) \geq 0$ for all $a_{W^{\prime}} \in A^{W^{\prime}}$. If $D\left(p\left(. \mid a_{W^{\prime}}\right) ; p\left(. \mid a_{W}\right)\right)=$ 0 for all $a_{W^{\prime}} \in A^{W^{\prime}}$ then $p\left(a \mid a_{W^{\prime}}\right)=p\left(a \mid a_{W}\right)$ for all $a_{W^{\prime}} \in A^{W^{\prime}}$ and all $a \in A$. On the other hand, as ne $(v)$ is a Markov neighborhood and ne $(v) \subset W^{\prime}$ we have that

$$
p\left(a \mid a_{W^{\prime}}\right)=p\left(a \mid a_{\mathrm{ne}(v)}\right) \text { for all } a_{W^{\prime}} \in A^{W^{\prime}} \text { and all } a \in A .
$$

Therefore we have that

$$
p\left(a \mid a_{W}\right)=p\left(a \mid a_{\mathrm{ne}(v)}\right) \text { for all } x_{W} \in A^{W} \text { and all } a \in A .
$$

By Lemma $4, W$ is a Markov neighborhood, i.e. $W \supset$ ne $(v)$, which contradicts the hypothesis in this case. So, $D\left(p\left(. \mid a_{W^{\prime}}\right) ; p\left(. \mid a_{W}\right)\right)>0$ for at least one $a_{W^{\prime}} \in A^{W^{\prime}}$ and then

$$
\sum_{a_{W^{\prime}} \in A^{W^{\prime}}} p\left(a_{W^{\prime}}\right) D\left(p\left(\cdot \mid a_{W^{\prime}}\right) ; p\left(\cdot \mid a_{W}\right)\right)>0 .
$$

Therefore

$$
\operatorname{PML}\left(x_{v}^{(1: n)} \mid x_{W^{\prime}}^{(1: n)}\right)>\operatorname{PML}\left(x_{v}^{(1: n)} \mid x_{W}^{(1: n)}\right)
$$

eventually almost surely as $n \rightarrow \infty$, finishing the proof of case (c).

With that, we proved that for any $W \subset V \backslash\{v\}$

$$
\operatorname{PML}_{\mathrm{ne}(v)}\left(x_{v}^{(1: n)} \mid x_{\mathrm{ne}(v)}^{(1: n)}\right)>\operatorname{PML}_{W}\left(x_{v}^{(1: n)} \mid x_{W}^{(1: n)}\right)
$$

eventually almost surely as $n \rightarrow \infty$, and as the set of possible subsets $W$ is finite we have

$$
\operatorname{PML}_{\mathrm{ne}(v)}\left(x_{v}^{(1: n)} \mid x_{\mathrm{ne}(v)}^{(1: n)}\right)>\max _{W \subset V \backslash\{v\}, W \neq \mathrm{ne}(v)}\left\{\operatorname{PML}_{W}\left(x_{v}^{(1: n)} \mid x_{W}^{(1: n)}\right)\right\}
$$

eventually almost surely as $n \rightarrow \infty$; that is, the estimator given in 2.20 satisfies $\widehat{\operatorname{ne}}(v)=\operatorname{ne}(v)$ eventually almost surely as $n \rightarrow \infty$.

Proof of Corollary 8. The proof of the corollary follows from Theorem 7, by noting that $V$ is finite. That is, for each vertex $v \in V$ we have that $\widehat{n e}(v)=$ ne $(v)$ eventually almost surely as $n \rightarrow \infty$, i.e., for each vertex $v \in V$ there exists a sample size $n(v)$ large enough such that with probability one, $\widehat{\mathrm{ne}}(v)=\operatorname{ne}(v)$ for all $n \geq n(v)$. As $V$ is finite we can take

$$
n^{*}=\max _{v \in V}\{n(v)\}
$$

then with probability one $\cap_{v \in V}\{\widehat{\operatorname{ne}}(v)=\operatorname{ne}(v)\}$ for all $n \geq n^{*}$ and this implies that, with probability one, $\widehat{G}^{-}=\widehat{G}^{+}=G$ for all $n \geq n^{*}$. 


\section{Chapter 3}

\section{Simulations}

This chapter presents some results for the examples in Chapter 2 througth simulations. First let's see how to generate samples from these examples, then how to use the estimation program. Next the results of the estimations will be evaluated from different perspectives: considering different values for the constant $c$ of the estimator and sample sizes.

\subsection{Generating samples}

For each example there is a different way to generate a sample from the Markov random field on $A^{V}$ with graph $G$. The simplest way to do that is using the factorized joint probability, i.e., using the conditional distributions insted of using the joing probability. Another knowledge required is how to generate a random variable, one way to do that is using the inverse transform method (see Rizzo (2007)). Now let's see each one of the algorithms, and the programs in R language ${ }^{1}$ that performs these algorithms are in Appendix B, Section B.1.

\subsubsection{Example 1}

This first example had its joint probability factorized like this (see 2.9):

$$
p\left(x_{1}, x_{2}, x_{3}, x_{4}, x_{5}\right)=p\left(x_{2} \mid x_{1}, x_{3}\right) p\left(x_{1} \mid x_{3}\right) p\left(x_{4} \mid x_{3}\right) p\left(x_{5} \mid x_{3}\right) p\left(x_{3}\right)
$$

So, to generate a sample from this Markov random field we follow this steps:

1. Generate the random variable $X_{3}$ from its marginal distribution;

2. Given $X_{3}=x_{3}$ in the first step, generate the random variables $X_{1}, X_{4}$ and $X_{5}$ from their conditional distributions $X_{i} \mid X_{3}=x_{3}, i=1,4,5$;

3. Given $X_{3}=x_{3}$ (step 1) and $X_{1}=x_{1}$ (step 2), generate the random variable $X_{2}$ from its conditional distribution $X_{2} \mid X_{1}=x_{1}, X_{3}=x_{3}$.

If it is wanted to generate a sample with size $n$, the steps 1-3 have to be repeated $n$ times.

\subsubsection{Example 2}

Now, this example has its joint probability factorized like this (see 2.13):

$$
p\left(x_{1}, x_{2}, x_{3}, x_{4}, x_{5}, x_{6}, x_{7}\right)=p\left(x_{1} \mid x_{2}\right) p\left(x_{2} \mid x_{3}\right) p\left(x_{4} \mid x_{3}\right) p\left(x_{3}\right) p\left(x_{5} \mid x_{6}\right) p\left(x_{7} \mid x_{6}\right) p\left(x_{6}\right)
$$

Then, the steps to generating a sample from this Markov random field are the following:

1. Generate the random variables $X_{3}$ and $X_{6}$ from their marginal distributions;

\footnotetext{
${ }^{1}$ www.r-project.org
} 
2. Given $X_{3}=x_{3}$ in the first step, generate the random variables $X_{1}, X_{2}$ and $X_{4}$ from their conditional distributions $X_{i} \mid X_{3}=x_{3}, i=1,2,4$, and given $X_{6}=x_{6}$ (step 1) generate the random variables $X_{5}$ and $X_{7}$ from their conditional distributions $X_{j} \mid X_{6}=x_{6}, j=5,7$.

To generate a sample with size $n$, just repeat the steps 1 and $2 n$ times.

\subsubsection{Example 3}

This example has its joint probability factorized like this (see 2.14):

$$
p\left(x_{1}, x_{2}, x_{3}, x_{4}, x_{5}, x_{6}, x_{7}, x_{8}, x_{9}, x_{10}, x_{11}, x_{12}\right)=p\left(x_{1}\right) \prod_{i=1}^{11} p\left(x_{i+1} \mid x_{i}\right)
$$

Keeping in mind that this example represents a Markov chain with order 1 the steps to generate a sample from this model are:

1. Generate the random variable $X_{1}$ from the initial distribution;

2. Given $X_{1}=x_{1}$ (step 1), generate the random variable $X_{2}$ through the transition matrix $\pi$ of the Markov chain;

3. Given $X_{2}=x_{2}$ (step 2), generate the random variable $X_{3}$ through the transition matrix $\pi$ of the Markov chain;

12. Given $X_{11}=x_{11}$ (step 11), generate the random variable $X_{12}$ through the transition matrix $\pi$ of the Markov chain.

And then, to generate a sample with size $n$ the steps 1-12 have to be repeated $n$ times.

\subsubsection{Example 4}

This last example has its joint probability factorized like this (see 2.16):

$$
\begin{aligned}
& p\left(x_{1}, x_{2}, x_{3}, x_{4}, x_{5}, x_{6}, x_{7}, x_{8}, x_{9}, x_{10}\right) \\
= & p\left(x_{1} \mid x_{2}\right) p\left(x_{2} \mid x_{4}\right) p\left(x_{3} \mid x_{4}\right) p\left(x_{5} \mid x_{4}\right) p\left(x_{6} \mid x_{4}\right) p\left(x_{4}\right) p\left(x_{7} \mid x_{10}\right) p\left(x_{8} \mid x_{9}, x_{10}\right) p\left(x_{9} \mid x_{10}\right) p\left(x_{10}\right)
\end{aligned}
$$

In this case, the steps to generate a sample are:

1. Generate the random variables $X_{4}$ and $X_{10}$ from their marginal distributions;

2. Given $X_{4}=x_{4}$ (step 1), generate the random variables $X_{1}, X_{2}, X_{3}$ and $X_{5}$ from their conditional distributions $X_{i} \mid X_{4}=x_{4}, i=1,2,3,5$, and given $X_{10}=x_{10}$ (step 1) generate the random variables $X_{7}$ and $X_{9}$ from their conditional distributions $X_{j} \mid X_{10}=x_{10}, j=7,9$;

3. Given $X_{10}=x_{10}$ (step 1) and $X_{9}=x_{9}$ (step 2), generate the random variable $X_{8}$ from its conditional distribution $X_{8} \mid X_{9}=x_{9}, X_{10}=x_{10}$.

Repeate the steps 1-3 $n$ times to have a sample with size $n$.

\subsection{How to use the estimation program}

With the help of the programmers at NUMEC-USP ${ }^{2}$ (Support Center for Stochastic Modeling and Complexity at Universtity of São Paulo), it was developed a program ${ }^{3}$, in C language, that,

\footnotetext{
${ }^{2}$ http://www.numec.prp.usp.br/

${ }^{3}$ available in http://github.com/yoshiomori/neighborhoods.git
} 
considering a sample, estimates the appropriate graph based on the estimator proposed in this thesis (Sections 2.4 and 2.5).

The program take into count informations:

a) the alphabet $A$;

b) the value $c$ of the estimator constant;

c) the sample;

d) the choice if the estimator should be conservative or not;

e) and the adjacency matrix (simetric matrix with zeros and ones, and diagonal of zeros) that gives the maximal neighborhood that should be tested.

The last item in this list, the adjacency matrix, it is useful when you have a prior knowledge about the neighborhoods, that is, when the absence of some of the edges is known, decreasing the number of comparisons made by the algorithm. If this information is unknown the adjacency matrix should represent the complete graph, i.e., to find the neighborhood of a vertex the algorithm will test all possible combinantions of the remaining vertices.

So, the program reads a file with the alphabet $A$, the value of $c$, the sample (a $|V| \times n$ matrix) and the adjacency matrix. The following presents an example of this file for an alphabet $A=(2,3)$, a $c=1.5$, a sample of $X=\left(X_{1}, X_{2}, X_{3}\right)$ of size 10 and an adjacency matrix of a complet graph:

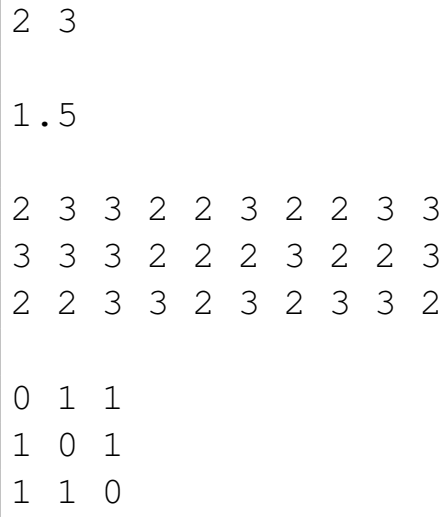

Given this file, the choice between a conservative or non conservative algorithm is made at the time of program execution, 0 (zero) represents the conservative choice and 1 (one) the non conservative one. And, as a result, the program gives two output files: a -neig.txt, that contains the adjacency matrix of the graph that was estimated; and a -prob.txt, that contains the estimates conditional probabilities of each vertex given its estimated neighborhood.

In the next section this program was used to evaluate the performance of the estimator proposed in this thesis, as well as used in Chapter 4 in the applications to real datasets.

\subsection{Estimator performance}

In this section the estimator's performance it is evaluated in three different ways. The first one considers samples of diferent sizes and estimate the graphs using different values of the constant $c$ with the two approaches (conservative and non conservative). In the second one, for a fixed value of $c$, the underestimation and overestimation errors are estimated, making it possible to evaluate their behavior with the growth of sample size. The last evaluation uses ROC (Receiver Operating Characteristic) curves (see Fawcett (2006)) to compare the conservative and non conservative approaches, for different values of c and a fixed sample size. 


\subsubsection{Evaluating convergence of the graphs}

Here just one sample of each sample size was generated to be used by the program with different values of $c$. There were generated sample of sizes: 100, 500, 1, 000, 5, 000 and 10,000, and the different values of $c$ used were: $0.25,0.5,1,1.5$ and 2 .
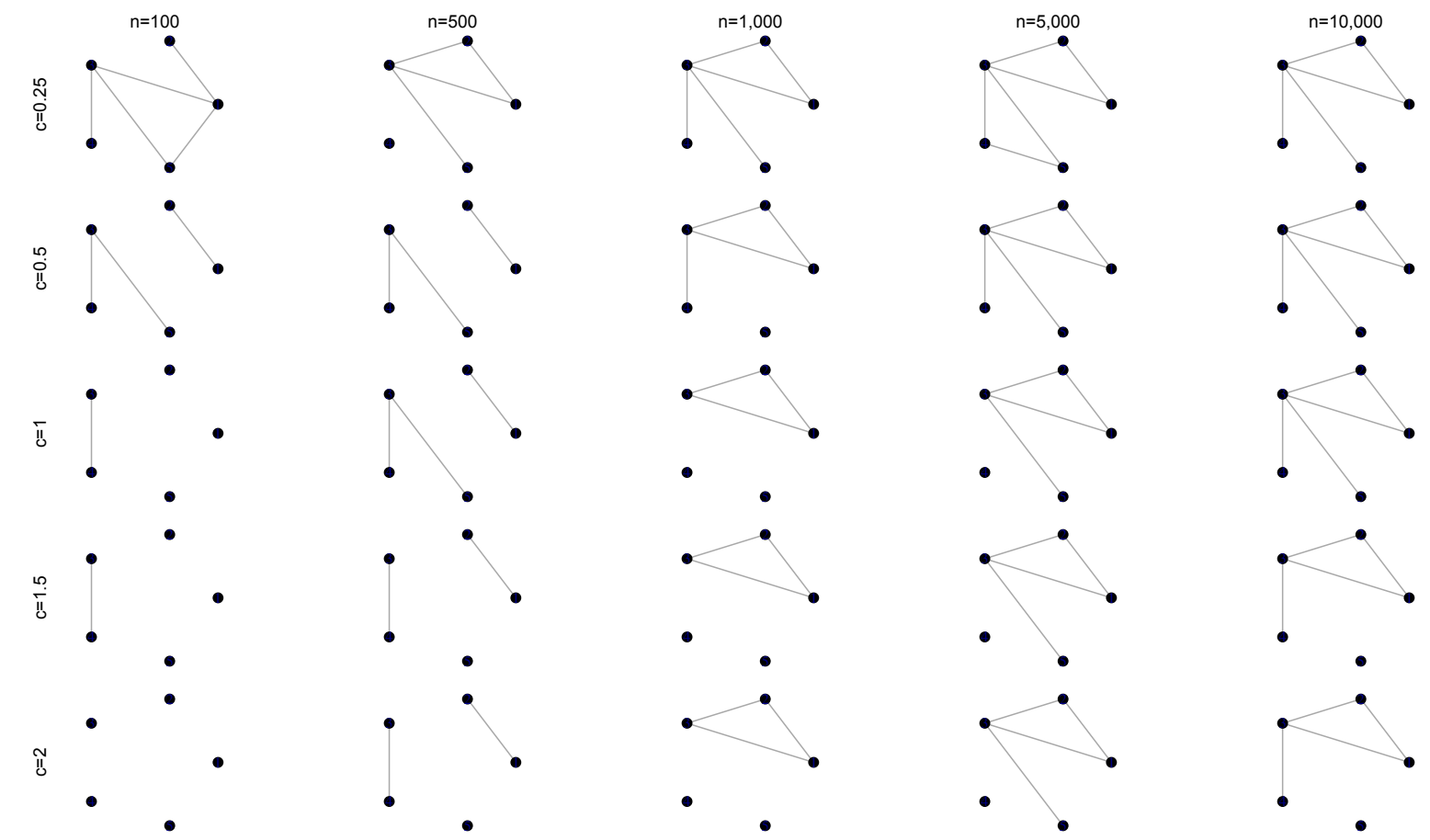

Figure 3.1: Graph of example 1: Evolution of the estimated graph for different sample sizes and constant $c$ considering the conservative option.
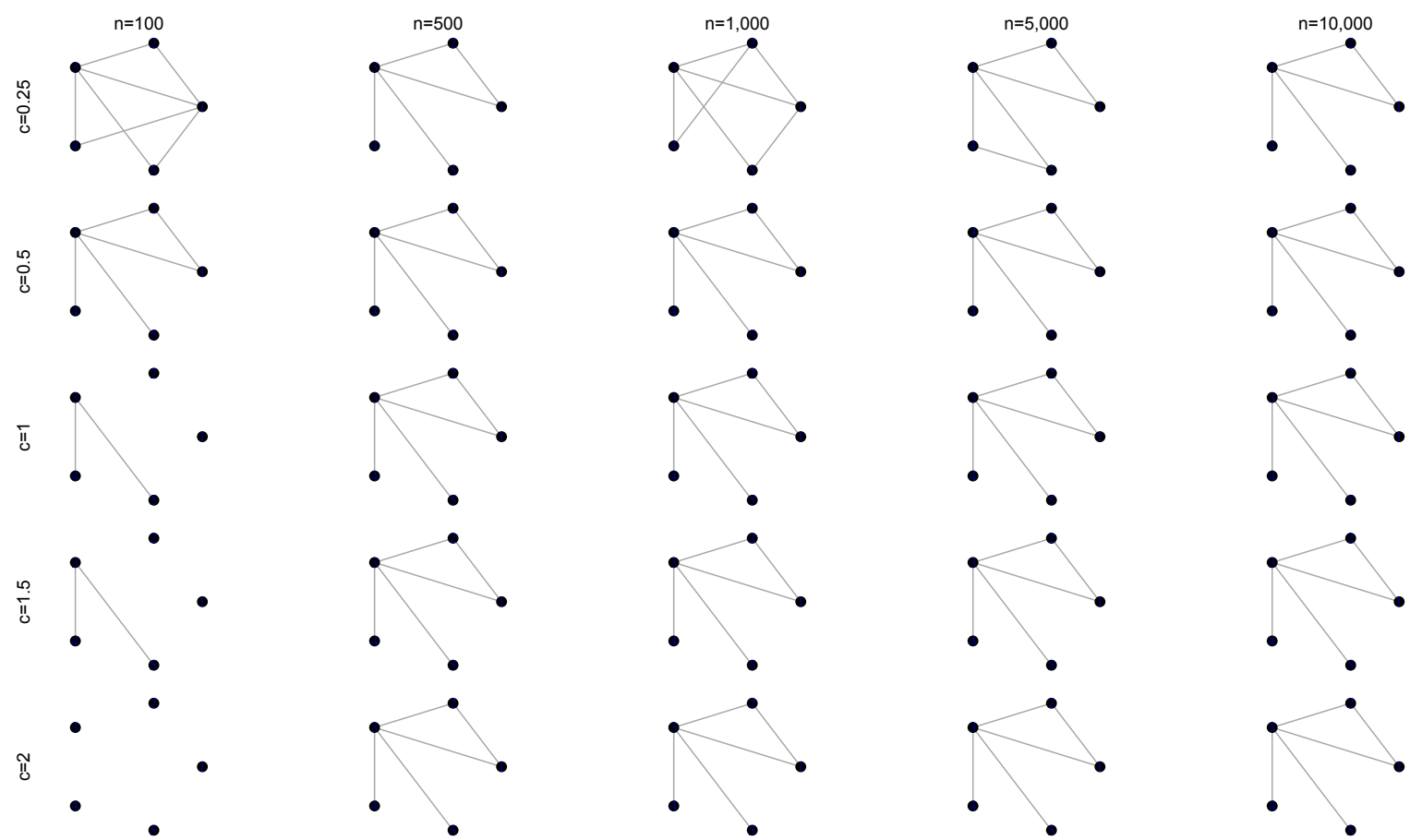

Figure 3.2: Graph of example 1: Evolution of the estimated graph for different sample sizes and constant c considering the non conservative option.

First they are presented the results of Example 1, Figures 3.1 and 3.2, considering the conservative and the non-conservative options, respectively.Compared to the results with the real graph, 
Figure 2.1 in Chapter 2, with the simulation results it is possible to see that:

- For the sample with size $n=100$, it wasn't possible to reconstruct the correct dependence structure of the graph for any of the values of $c$ in the conservative option (Figure 3.1, first column), for $c=0.5,1,1.5$ and 2 the graph was underestimated, and for $c=0.25$ there is one missing edge and one spare edge. But for the non conservative option (Figure 3.2, first column) the correct dependence structue was found just for $c=0.5$, for $c=0.25$ there are two spares edges, and for the other values of $c$ (the highest values) the graph was underestimated.

- For the sample with size $n=500$, again it wasn't possible to reconstruct the correct dependence estructure of the graph for any of the values of $c$ in the conservative option (Figure 3.1 , second column), but in this case for all values of $c$ the graph was underestimated. On the other hand, for the conservative option (Figure 3.2, second column) the graph was correctly estimated for all values of $c$.

- For the sample with size $n=1,000$, in the conservative option (Figure 3.1, third column) just for $c=0.25$ the graph was correctly estimated, for the other values of $c$ the graph was underestimated. The opposite occurred in the non conservative option (Figure 3.2, third column), here just for $c=0.25$ the graph wasn't correctly estimated, it was overestimated with two spare edges, and for the other values of $c$ the graph was correctly estimated.

- For the sample with size $n=5,000$, in the conservative option (Figure 3.1, fourth column) the graph was overestimated for $c=0.25$ with one spare edge, it was correctly estimated for $c=0.5$, and it was uderestimated for the other ones. In the non conservative option (Figure 3.2 , fourth column), like in the other option, the graph was overestimated for $c=0.25$, but it was correctly estimated for all the other values of $c$.

- And for the sample with size $n=10,000$, in the conservative option (Figure 3.1, fifth column) the graph was correctly estimated for $c=0.25,0.5$ and 1 , and it was underestimated for $c=1.5$ and 2 . In the non conservative option (Figure 3.2, fifth column), the graph was correctly estimated for all values of $c$.

In general, for this example the non conservative approach was better then the conservative one, being able to properly estimate the dependece structure of the graph 19 of 25 times against 5 of 25 times.

Now the results for Example 2 are presented in Figures 3.3, the conservative option, and Figure 3.4, the non conservative option. Comparing these results with the correct dependence estructure of the graph in Figure 2.2 (Chapter 2) it is possible to observe that:

- For the sample with size $n=100$ the conservative option (Figure 3.3, first column) and the non conservative option (Figure 3.4, first column) were not capable of reconstructing the true graph structure. In the conservative option for $c=0.25$ there is two spare edges and three missing edges, for the other values of $c$ there is just missing edges. In the non conservative option for $c=0.25$ there is two spare edges and two missing edges, for $c=0.5$ there is two spare edges and three missing ones, and for the other values there are just missing edges.

- For the sample with size $n=500$ again none of the options were able of reconstructing the true graph structure. In the conservative option (Figure 3.3, second column) the graph was underestimated for all values of $c$. In the non conservative option (Figure 3.4, second column) the graph was not properly estimated for any value of $c$, for $c=0.25$ and 0.5 there is one spare edge and two missing edges, for the other values of $c$ there are just missing edges.

- For the sample with size $n=1,000$, in the conservative option (Figure 3.3, third column) the graph was underestimated for all values of $c$. But in the non conservative option (Figure 3.4, third column) the graph was correctly estimated only for $c=0.25$, for the other values of $c$ the graph was underestimated. 

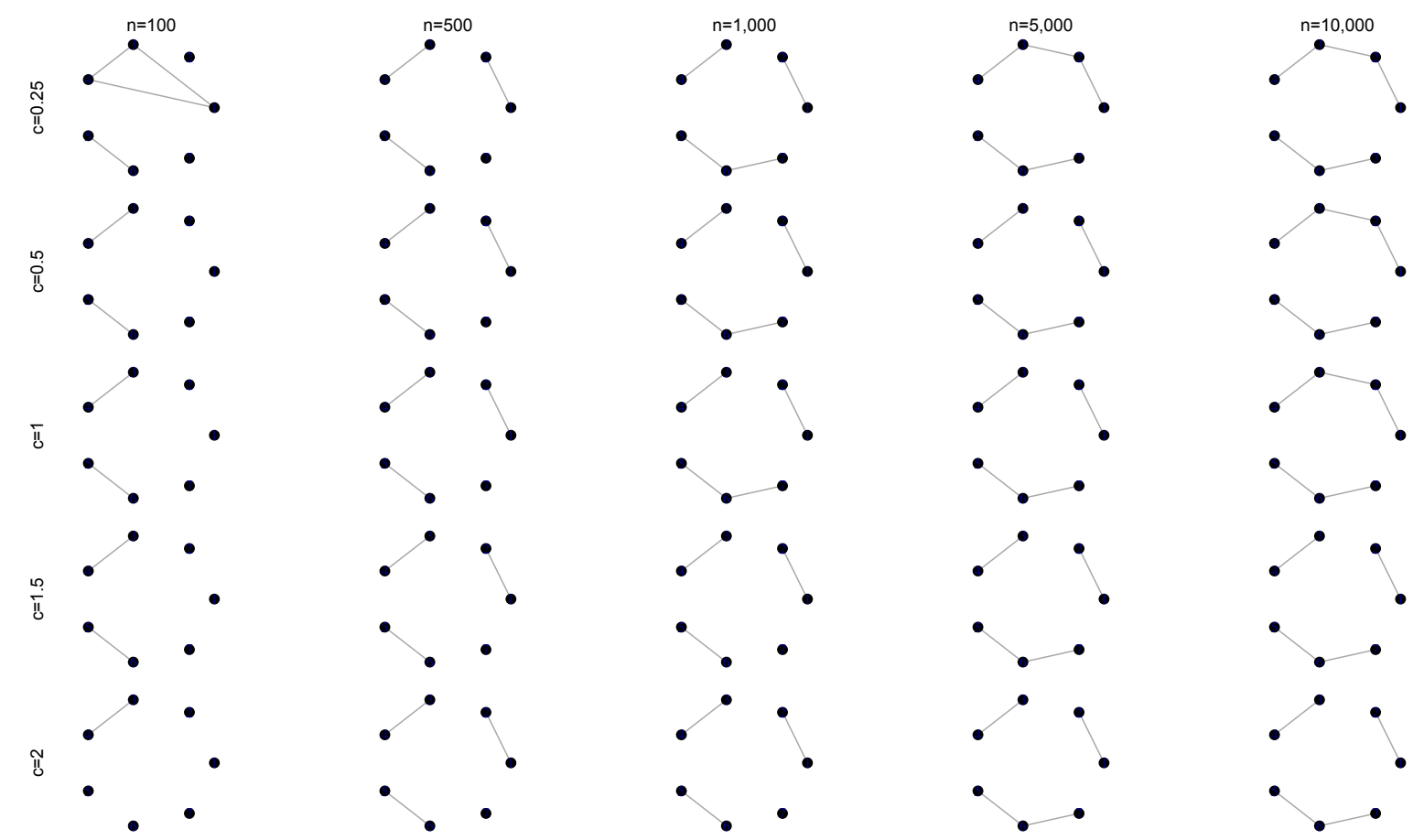

Figure 3.3: Graph of example 2: Evolution of the estimated graph for different sample sizes and constant $c$ considering the conservative option.
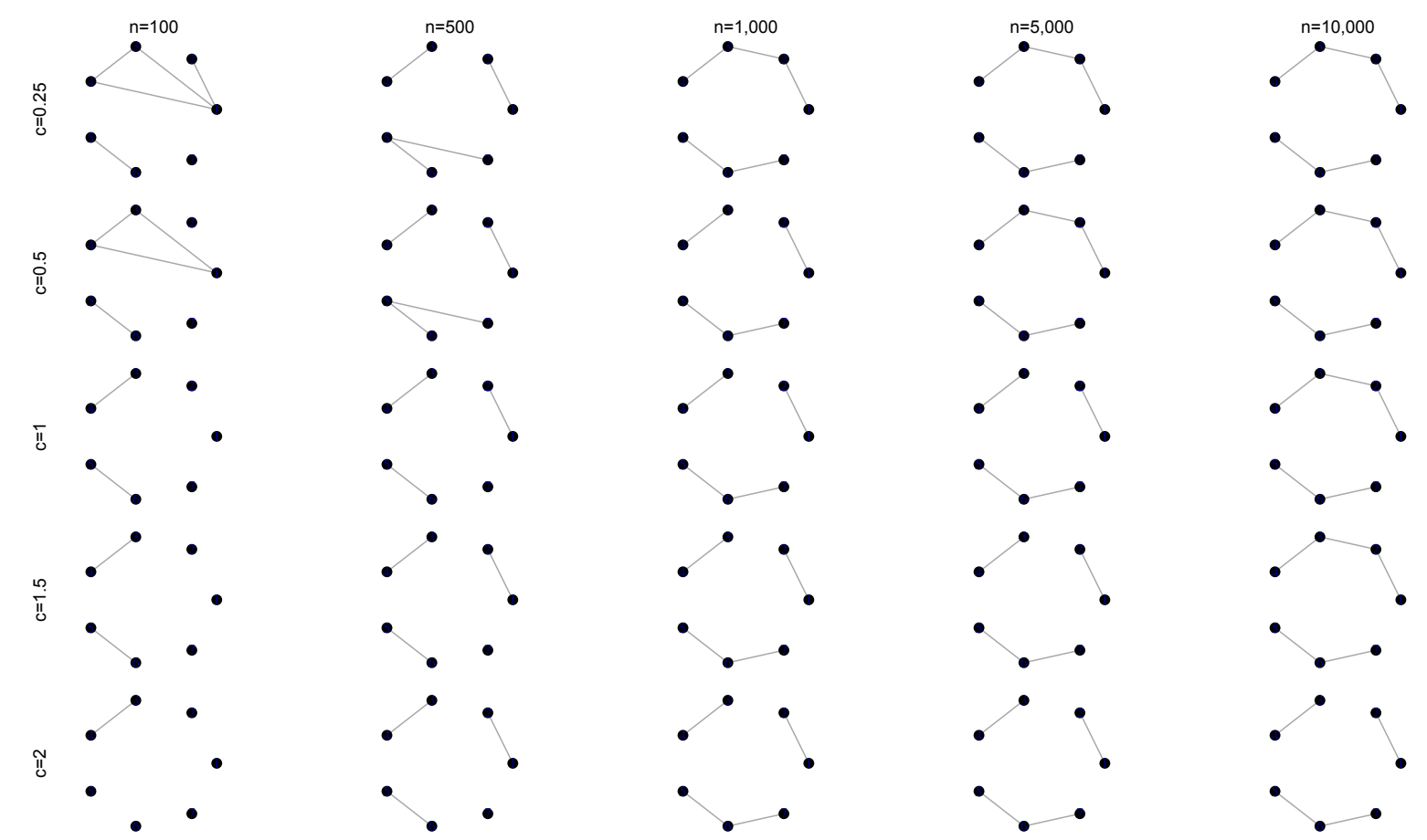

Figure 3.4: Graph of example 2: Evolution of the estimated graph for different sample sizes and constant $c$ considering the non conservative option.

- For the sample with size $n=5,000$, in the conservative option (Figure 3.3, fourth column) the graph was correctly estimated for $c=0.25$, for the other values of $c$ the graph was underestimated. In the non conservative option (Figure 3.4, fourth column) the graph was correctly estimated for $c=0.25$ and 0.5 , and it was underestimated for the other values of $c$.

- For the sample with size $n=10,000$, in the conservative option (Figure 3.3, fifth column) the graph was correctly estimated for the first three values os $c(0.25,0.5$ and 1$)$ and it was 
underestimated for the other values. Now in the non conservative option (Figure 3.4, fifth column), just for $c=2$ the graph was not correctly estimated, it was underestimated, for the other values of $c$ it was correctly estimated.

Like in the first example, for Example 2, the non conservative approach was slightly better than the conservative one considering the sample sizes and values of $c$, having correctly estimated the graph 6 of 25 times against 4 of 25 times.
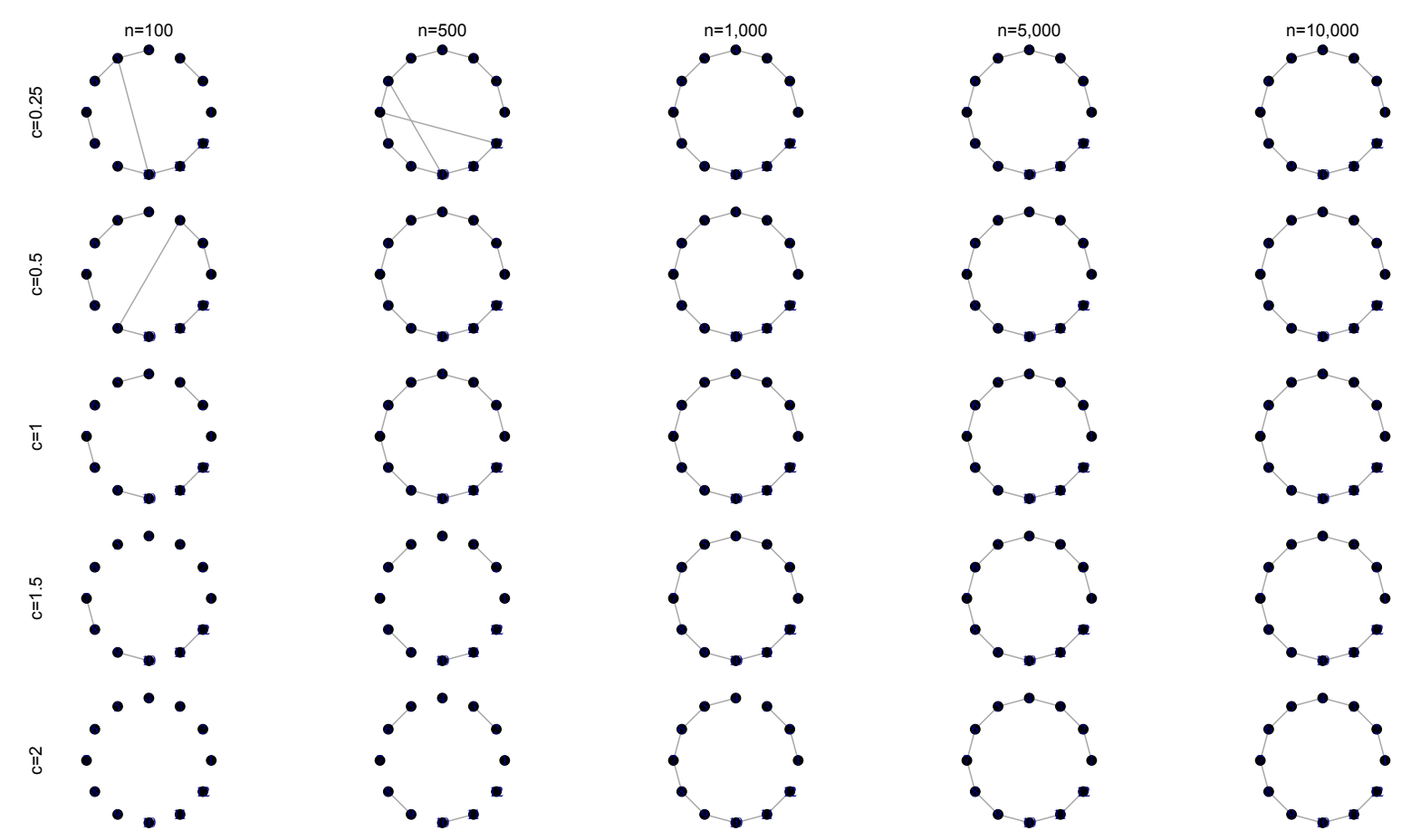

Figure 3.5: Graph of example 3: Evolution of the estimated graph for different sample sizes and constant $c$ considering the conservative option.
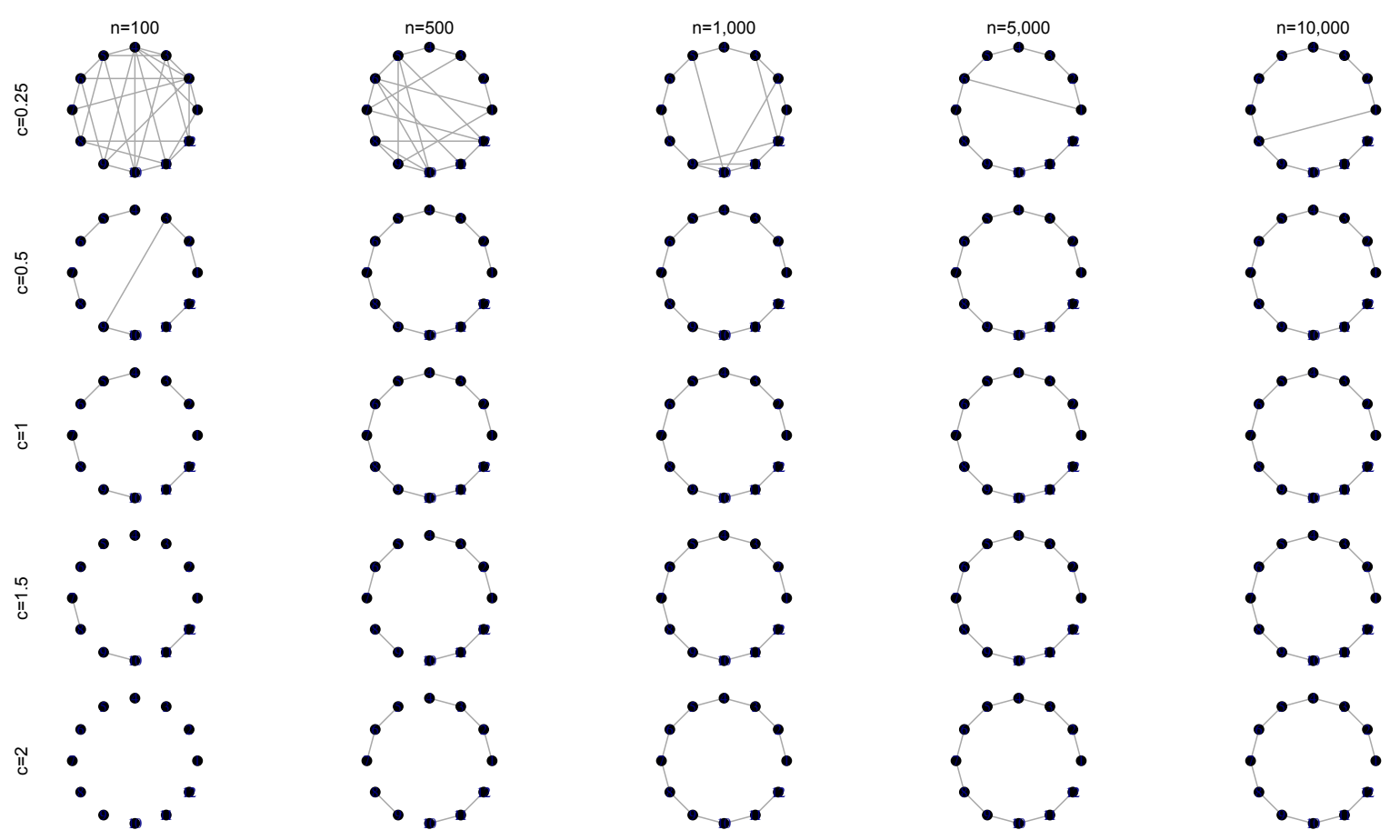

Figure 3.6: Graph of example 3: Evolution of the estimated graph for different sample sizes and constant $c$ considering the non conservative option. 
For example 3 the results are presented in Figures 3.5 and 3.6, the conservative and non conservative options respectively. Comparing the results with the correct dependence structure of the graph in Figure 2.3 (Chapter 2) it is possible to see that:

- For the sample with size $n=100$ the conservative option (Figure 3.3, first column) and the the non conservative option (Figure 3.4, first column) were not capable of reconstructing the true graph structure. The biggest difference between the two options is for $c=0.25$, note that for the conservative option there is only one spare edge and for the non conservative one there are 15 spare edges.

- For the sample with size $n=500$, for the conservative option (Figure 3.3, second column) the graph was overestimated for $c=0.25$, it was correctly estimated for $c=0.5$ and 1 , and it was underestimated for the other values of $c$. The same happened for the non conservative option (Figure 3.4, second column), but for $c=0.25$ the number of spare edges was bigger (two against ten edges).

- For the sample with size $n=1,000$, for the conservative option (Figure 3.3, third column) the graph was correctly estimated for $c=0.25,0.5,1$ and 1.5, and it was underestimated, with one missing edge, for $c=2$. For the non conservative option (Figure 3.4, third column) the graph was correctly estimated for $c=0.5,1,1.5$ and 2 , and it was overestimated, with six spare edges for $c=0.25$.

- For the samples with sizes $n=5,000$ and $n=10,000$, for the conservative option (Figure 3.3, fourth and fifth columns) the graph was correctly estimated for all values of $c$. And for the non conservative option (Figure 3.4, fourth and fifth columns) the graph was correctly estimated for $c=0.5,1,1.5$ and 2 , and it was overestimated, with one spare edge, for $c=0.25$.

Unlike what happened in the two previous examples, in this one the conservative option was slightly better than the non conservative one, having correctly estimated the graph 16 of 25 times against 14 of 25 .
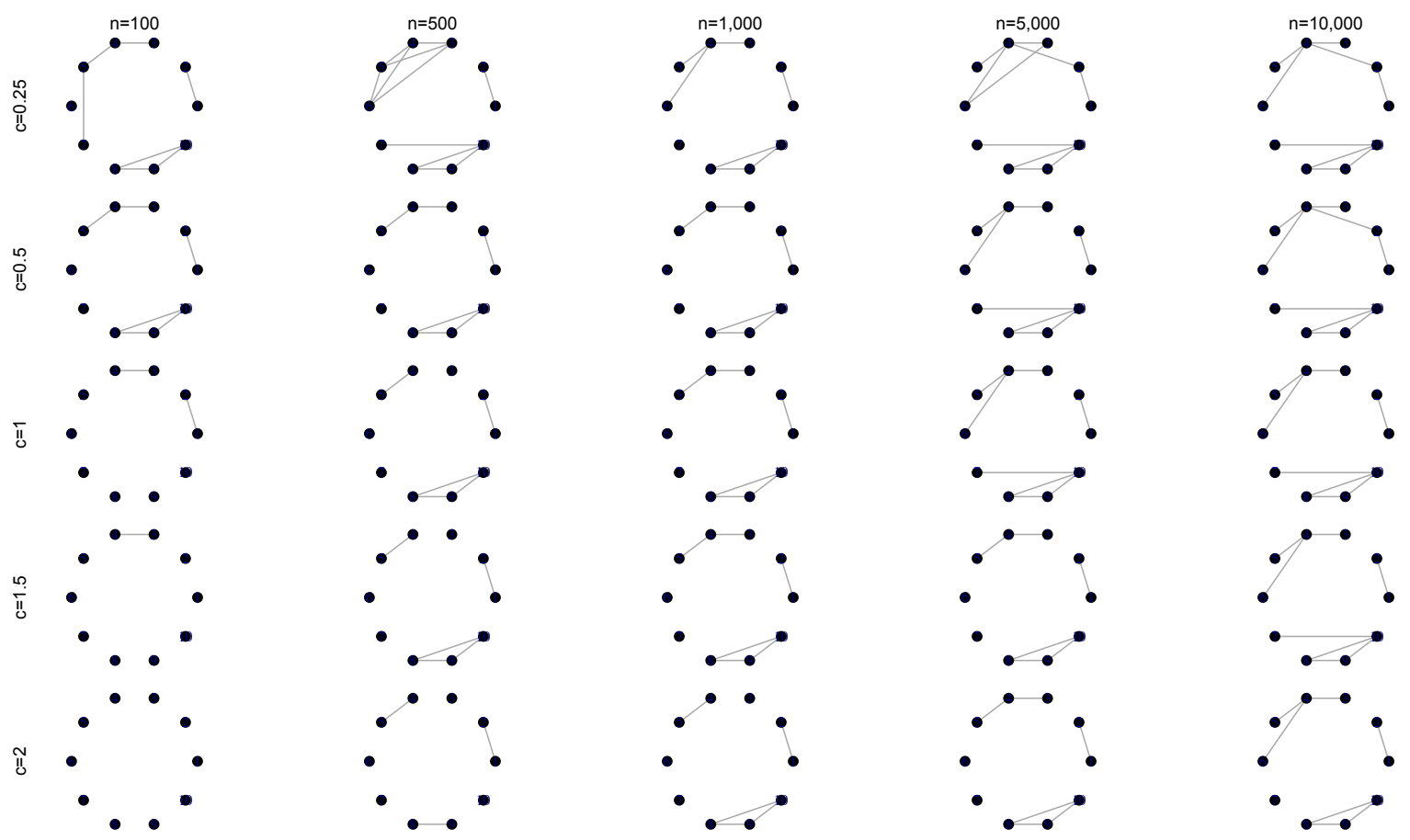

Figure 3.7: Graph of example 4: Evolution of the estimated graph for different sample sizes and constant $c$ considering the conservative option. 

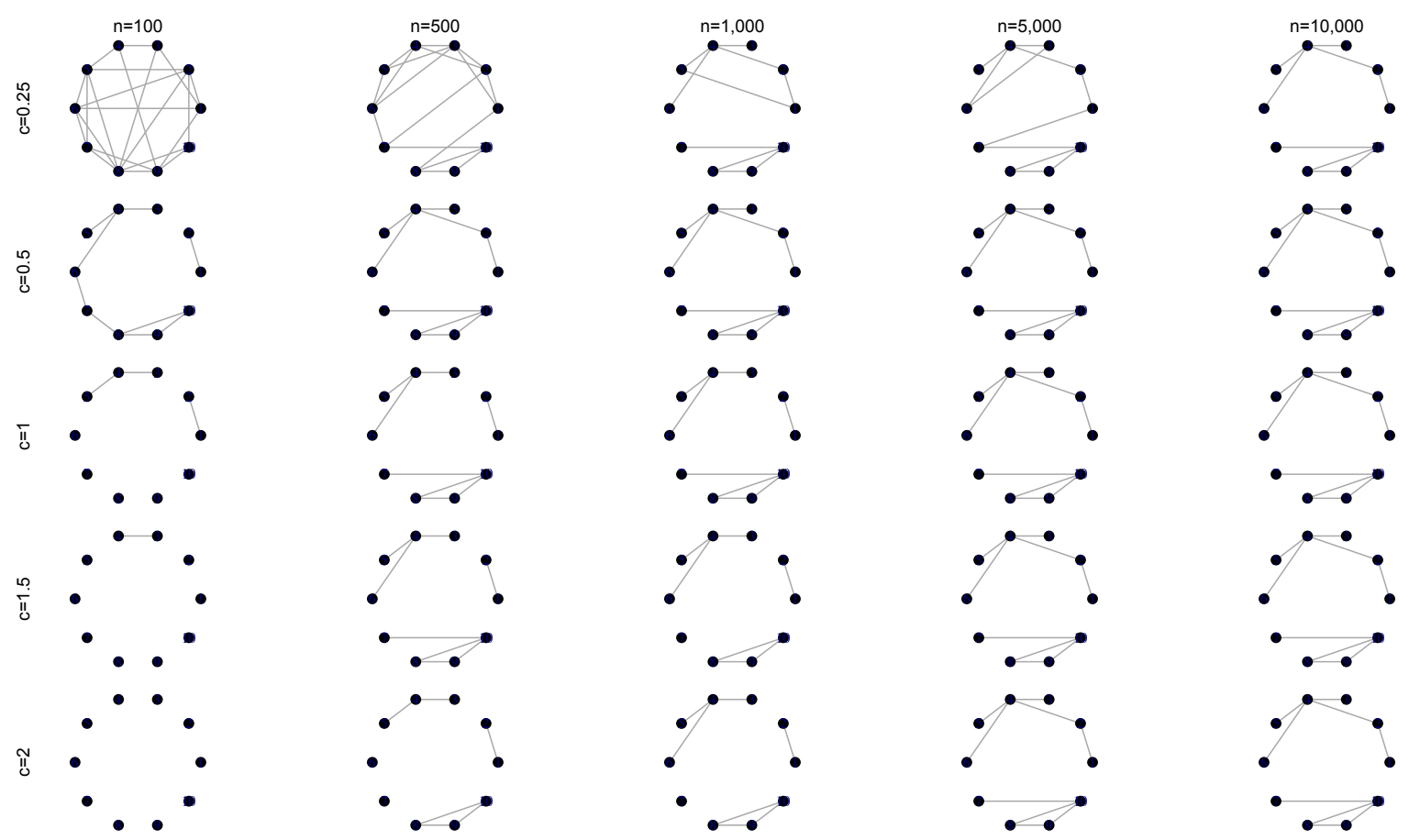

Figure 3.8: Graph of example 4: Evolution of the estimated graph for different sample sizes and constant $c$ considering the non conservative option.

The results of the last example, Example 4, are presented in Figures 3.7, the conservative option, and Figure 3.8, the non conservative option. Compared to Figure 2.4 in Chapter 2, that contains the real structure of the graph, with the results presented here it is possible to observe that:

- For the sample with size $n=100$, in the conservative option (Figure 3.1, first column) the graph was underestimated for all values of $c$. Note that for $c=2$ there are no edges, this kind of graph means that all variables are independent. And in the non conservative option (Figure 3.1, first column) the graph was overestimated for $c=0.25$, for $c=0.5$ there is two missing edges and two spare edges, for the other values of $c$ the graph was underestimated. As in the other option, here for $c=2$ the graph has no edges. None of the options were able to estimate the correct graph structure.

- For the sample with size $n=500$, in the conservative option (Figure 3.7, second column) for all values of $c$ the graph was not correctly estimated, for $c=0.25$ there are missing and spare edges, and for the other values of $c$ the graph was underestimated. In the non conservative option (Figure 3.8, second column) the graph was correctly estimated only for $c=0.5$, it was overestimated for $c=0.25$ and underestimated for $c=1.5,1$ and 2 .

- For the sample size $n=1,000$, in the conservative option (Figure 3.7, third column) the graph was underestimated for all values of $c$. And in the non conservative option (Figure 3.8, third column), the graph was correctly estimated only for $c=0.5$, it was overestimated for $c=0.25$, with one spare edge, and it was underestimated for the other values of $c$.

- For the sample size $n=5,000$, in the conservative option (Figure 3.7, fourth column) the graph was overestimated for $c=0.25$ and it was underestimated for the other values of $c$. In the non conservative option (Figure 3.8, fourth column) the graph was correctly estimated for $c=0.5,1,1.5$ and 2 , and it was overestimated for $c=0.25$.

- For the sample size $n=10,000$, in the conservative option (Figure 3.7, fifth column) the graph was correctly estimated for $c=0.25$ and 0.5 , and it was underestimated for the other values of $c$. And in the non conservative option (Figure 3.8, fifth column) the graph was correctly estimated for all values of $c$. 
For this example, as in Examples 1 and 2, the non conservative approach seems to be better than the conservative one, having correctly estimated the graph 11 of 25 times against 2 of 25 times.

Throughout the examples it is possible to notice the effect of the sample size, the choice of the estimator constant $c$ and if the estimator must be conservative or not. In summary, the increase of the sample size improves the estimation of the graph; the higher the value of the constant more evidence is needed to perceive the existence of edges, i.e., the choice of the constant makes the estimator more or less conservative; and even the choice between a conservative estimator or not impacts on the perception of the edges, a conservative estimator needs more information to put an edge between two vertices.

\subsubsection{Under and Overestimation errors}

In this evaluation it was used a constant $c=1$, and the sample size ranged from 100 to 10,000 . To estimate the errors, for each sample size they were generated 20 samples, and then it was calculated the average of the errors. The understimation error $(u e)$ is given by the number of missing edges over the number of edges that the graph has, the overestimation error (oe) is given by the number of spare edges over the number of edges that the graph does not have, and a total error $(t e)$ is given by the sum of missing edges and spare edges over the total number of edges of the complete graph. That is, let $\widehat{G}=(V, \widehat{E})$ be an estimative of the graph $G=(V, E)$, then:

$$
\begin{gathered}
u e=\frac{\sum_{v \in V} \sum_{v<w} \mathbf{1}\{(v, w) \in E \text { and }(v, w) \notin \widehat{E}\}}{\sum_{v \in V} \sum_{v<w} \mathbf{1}\{(v, w) \in E\}}, \\
o e=\frac{\sum_{v \in V} \sum_{v<w} \mathbf{1}\{(v, w) \notin E \text { and }(v, w) \in \widehat{E}\}}{\sum_{v \in V} \sum_{v<w} \mathbf{1}\{(v, w) \notin E\}} \text { and } \\
t e=\frac{\sum_{v \in V} \sum_{v<w}(\mathbf{1}\{(v, w) \in E \text { and }(v, w) \notin \widehat{E}\}+\mathbf{1}\{(v, w) \notin E \text { and }(v, w) \in \widehat{E}\})}{\frac{|V|(|V|-1)}{2}} .
\end{gathered}
$$
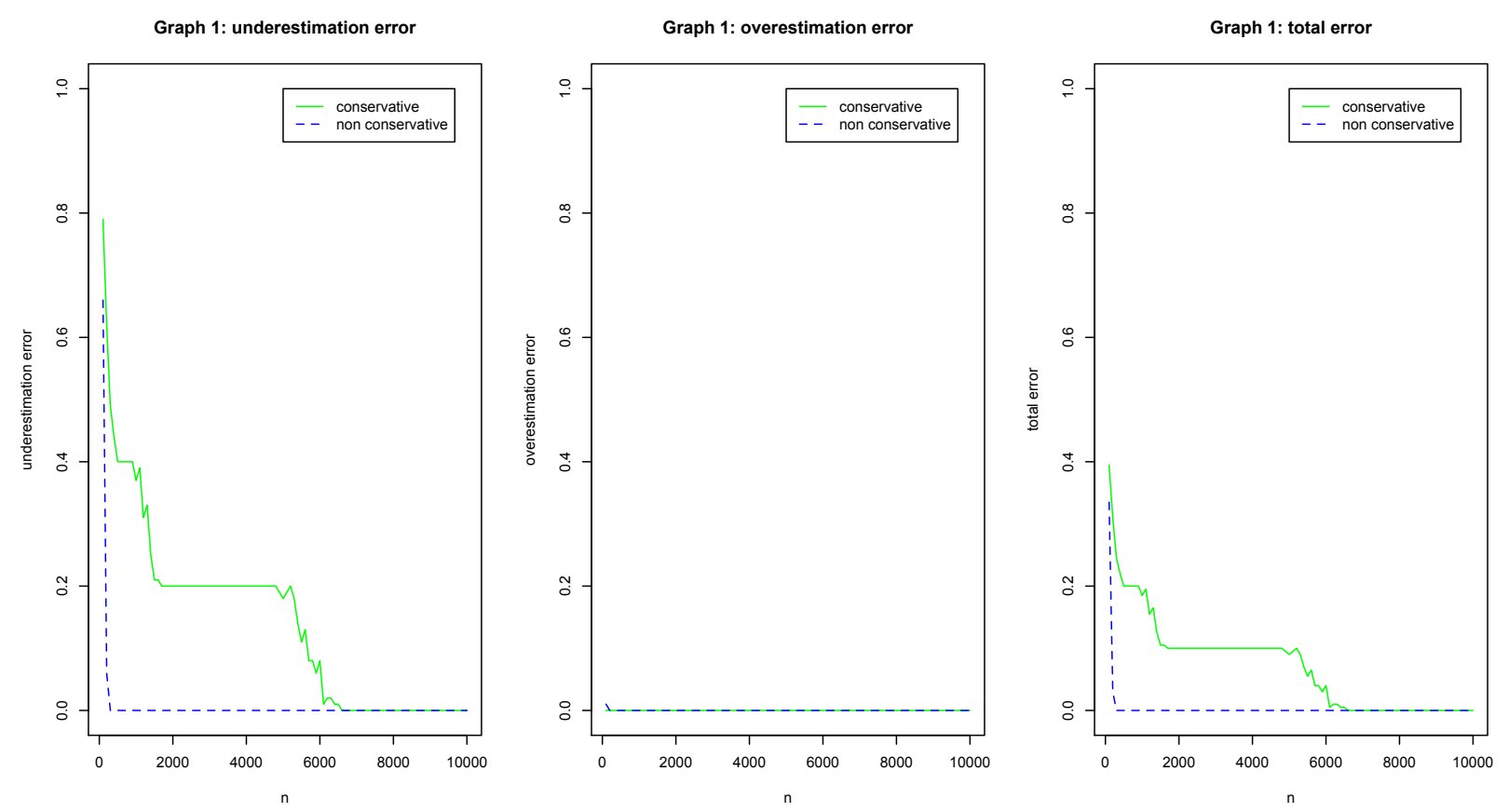

Figure 3.9: Graph of example 1: Estimative of the underestimation, overestimantion and total errors for different sample sizes with $c=1$.

The comparison of the different types of errors was made for each one of the four examples we 
are analyzing, and the results can be observed in Figures 3.9, 3.10, 3.11 and 3.12. And to calculate the errors the function errors in B.2 written in R language can be used.

For the first example, Figure 3.9, it is possible to see how the underestimation error of the non conservative (blue dashed line) choice decreases faster than the error of the conservative one (green continuous line). The overstimation errors were almost zero even for small sample sizes and in both cases (conservative and non conservative choices), and because of this, the total errors reflects only the behavior of the underestimation errors. So, for this example the non consevative seems to be the best choice.
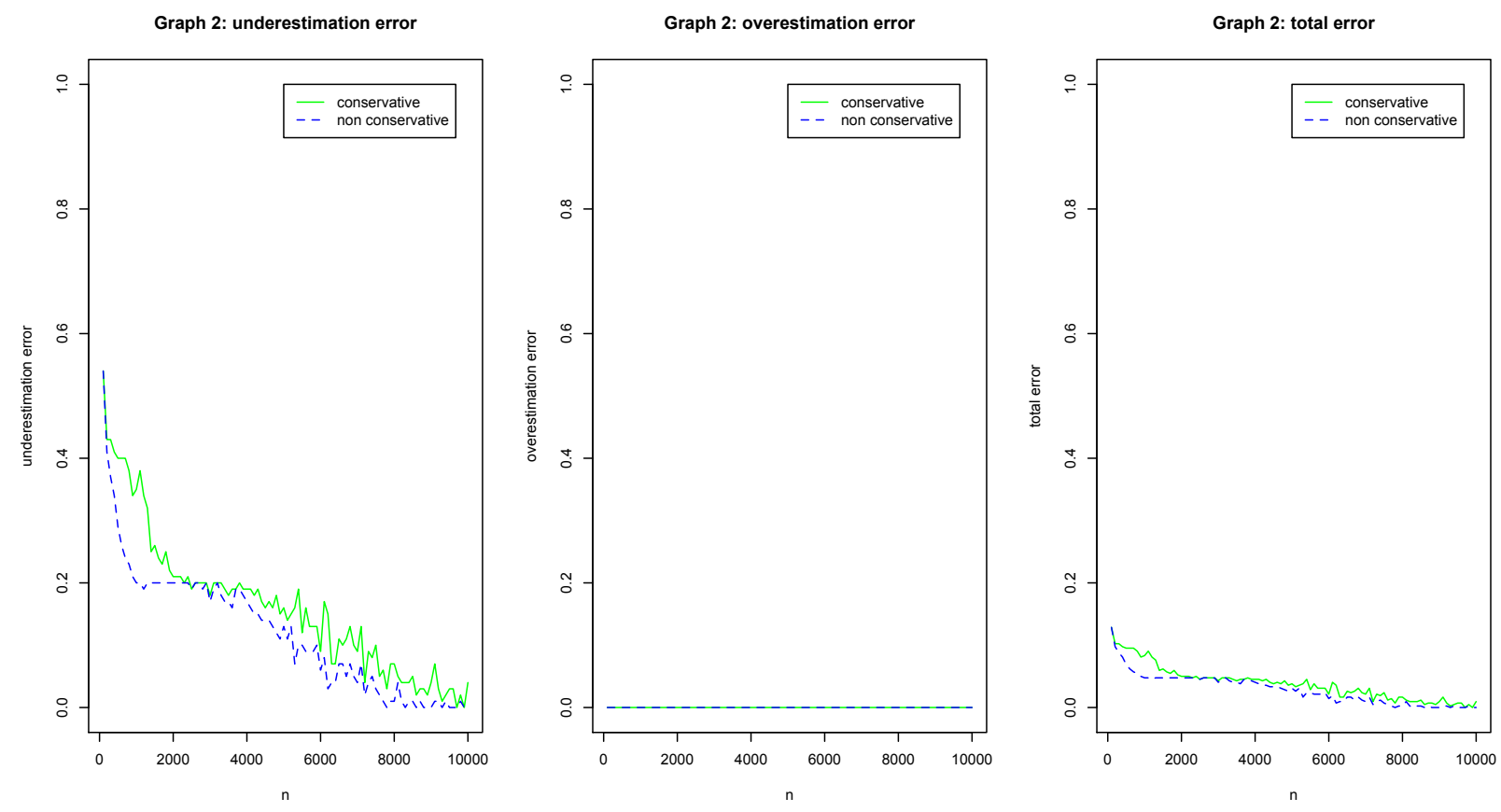

Figure 3.10: Graph of example 2: Estimative of the underestimation, overestimantion and total errors for different sample sizes with $c=1$.

Looking at the graphics in Figure 3.10, the results for the second example, we can see again that even for small sample sizes the overestimation errors in both cases are zero, and despite the underestimation errors being closer in this example, the errors of the non conservative choice are smaller than the errors of the conservative one. So, again the non conservative choice seems to be the best choice.

For the third example, Figure 3.11, the understimation errors decrease really fast with the increasing sample size, but again the non conservative choice has a better performance than the conservative one. Because the overestimation errors were zero for both choices the total errors have the same behavior of the underestimation errors. In conclusion, the non conservative choice proved to be the best choice.

In the last example, Figure 3.12, we have the same results for the overestimation errors for both choices, the errors are zero, and looking at the underestimation errors the non conservative choice has a much better performance than the conservative one.

We should keep in mind that the errors are affected not only by the sample size but also by the choice of the constant $c$. It seems that the value 1 for the constant is large enough to prevent the overestimation of edges. Perhaps with a lower value ofthe constant $c$ the overestimation errors could be observed, but this also would make the underestimation errors to be smaller.

\subsubsection{ROC curves}

The ROC (Receiver operating characteristic) curve was develop during World War II to detect electronic signals and problems with radars (Zweig and Campbell, 1993). Its goal was to quantify 

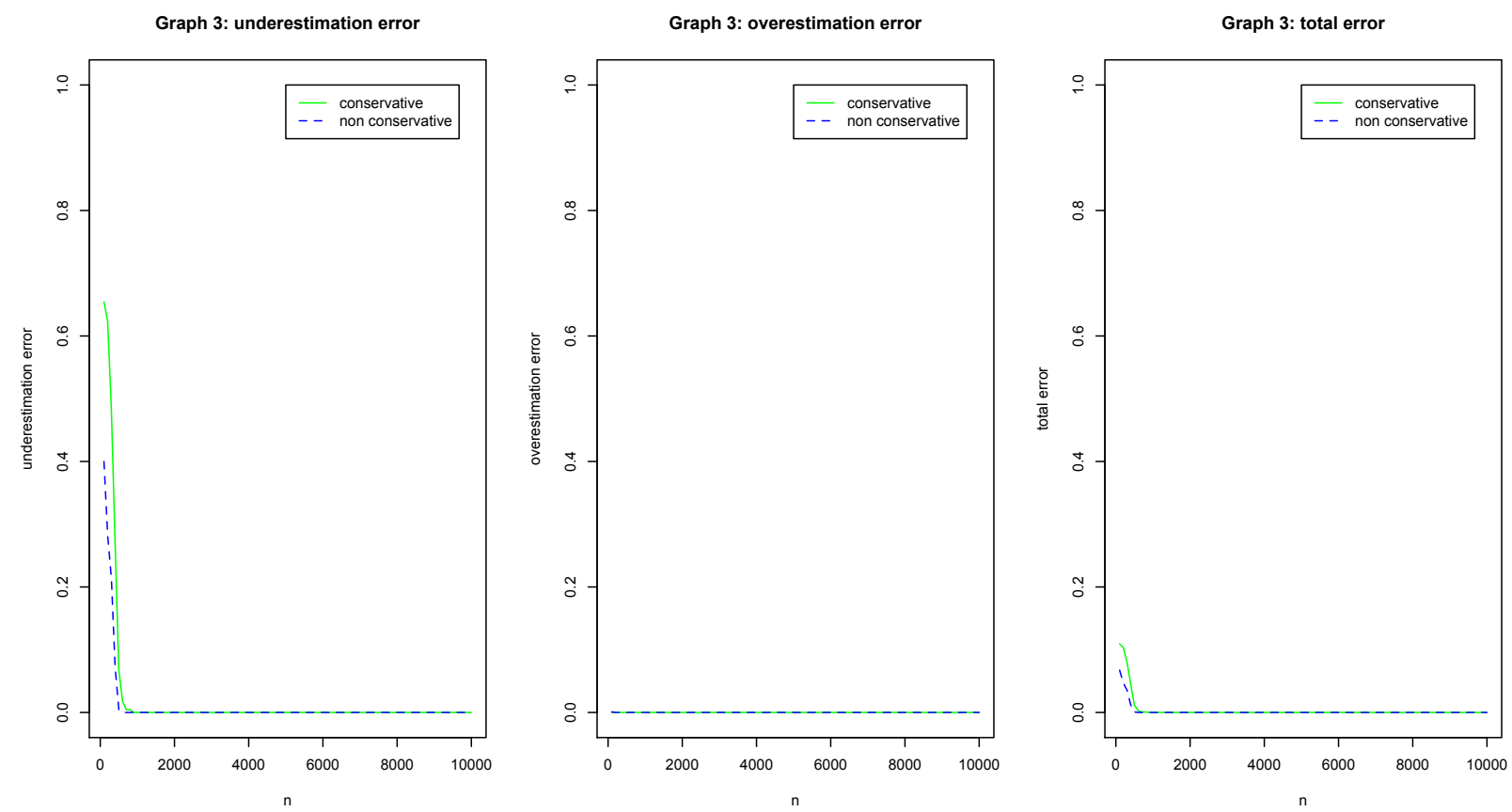

Figure 3.11: Graph of example 3: Estimative of the underestimation, overestimantion and total errors for different sample sizes with $c=1$.
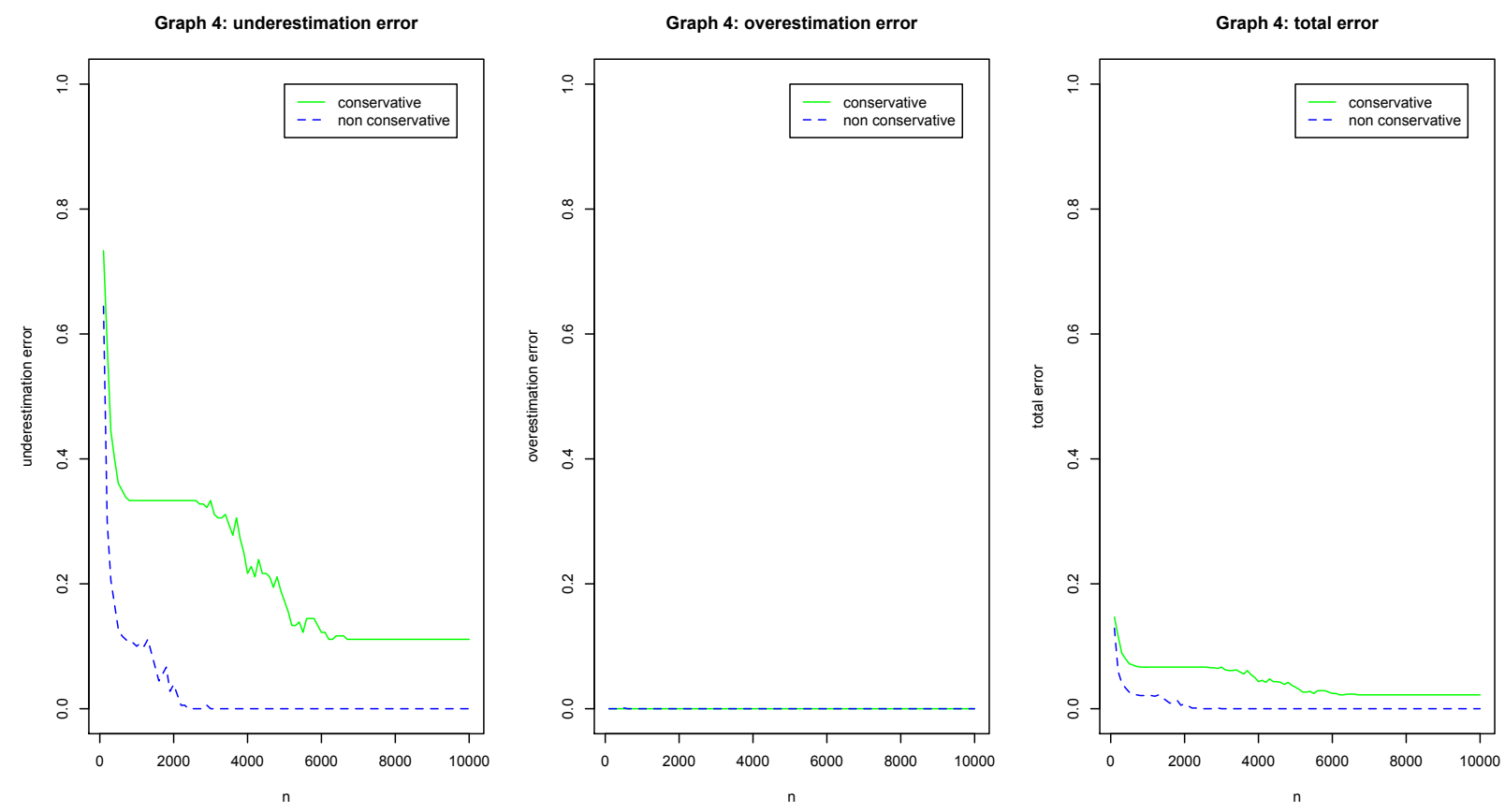

Figure 3.12: Graph of example 4: Estimative of the underestimation, overestimantion and total errors for different sample sizes with $c=1$.

the ability of radar operators (originally called receiver operators) to distinguish a signal from a noise (Reiser and Faraggi, 1997). This ability was called receiver operating characteristic (ROC). When the radar detected something approaching, it was up to the operator to decide whether what was captured was, for example, an enemy aircraft (the signal), or some other irrelevant flying object, like a flock of birds (the noise) (Collinson, 1998). In the 1960's, ROC curves were used in experimental psychology and, in the 1970's, the methodology has spread widely in various fields of biomedical research, an area in which the goal became basically to assist the classification of 


\begin{tabular}{|c|c|c|c|}
\hline \multirow[b]{2}{*}{ Graph } & \multicolumn{2}{|c|}{ Neighborhood estimator } & \\
\hline & Identifies the edge & $\begin{array}{l}\text { Does not identify the } \\
\text { edge }\end{array}$ & \\
\hline Has the edge & & False negative & $\begin{array}{l}\text { Number of existing } \\
\text { edges }\end{array}$ \\
\hline $\begin{array}{l}\text { Does not have the } \\
\text { edge }\end{array}$ & False positive & True negative & $\begin{array}{l}\text { Number of non exist- } \\
\text { ing edges }\end{array}$ \\
\hline
\end{tabular}

Table 3.1: Confusion matrix

individuals in sick or not sick. In our context, the neighborhood estimator proposed here acts as a classifier that identifies the existence of edges between two vertices.

Translating the concepts to our case, to construct the ROC curve two definitions must be understood: the sensibility, defined as the probability of identifying an edge given that the edge exists, also called as true positive rate; and the specificity, defined as the probability of do not identifying an edge given that the edge does not exist, and 1- specificity is also known as false positive rate. The ROC curve of a classifier is the pair (1- specificity, sensibility).

The ideal is to have a curve above the line represented by $y=x$, i.e., a classifier that has sensibility greater than $1-$ specificity. And when comparing the curves of two or more classifiers, if one curve dominate the others the classifier represented by this dominant curve is considered better than the others. But when this dominance is not clear, the choose of a better classifier should consider other factors that may be theoretical and/or practical.

The confusion matrix, in Table 3.1, is a common representation of the results of a classification method which contains the successes and failures of a rating. Now, let $\widehat{G}=(V, \widehat{E})$ be an estimative of the graph $G=(V, E)$, then:

$$
\text { Sensibility }(\widehat{G})=\frac{\text { True positive }}{\text { Number of existing edges }}=\frac{\sum_{v \in V} \sum_{v<w} \mathbf{1}\{(v, w) \in E \text { and }(v, w) \in \widehat{E}\}}{\sum_{v \in V} \sum_{v<w} \mathbf{1}\{(v, w) \in E\}} \text { and }
$$

$1-$ Specificity $(\widehat{G})=\frac{\text { False positive }}{\text { Number of non existing edges }}=\frac{\sum_{v \in V} \sum_{v<w} \mathbf{1}\{(v, w) \notin E \text { and }(v, w) \in \widehat{E}\}}{\sum_{v \in V} \sum_{v<w} \mathbf{1}\{(v, w) \notin E\}}$.

This pair can be calculated using the function roc.curve in B.2 written in R language.

To compare the conservative and non conservative approaches of the estimator using the ROC curve, they were used different values of the constant $c$, in such a way that each point of the curve was estimated by the average of the pair (1- specificity, sensibility) given by 50 samples for a particular value of $c$. The sample size was kept the same $(n=100)$, so the only thing that varies is the constant $c$ ranging from 0 to 3.5 .

Figures 3.13, 3.15 and 3.16, containing the results of Examples 1, 3 and 4, respectively, clearly show the dominance of the non-conservative estimator (blue asterisks) over the conservative estimator (green dots) resulting in a better classifier. In Figure 3.14, containing the results of Example 2 , this dominance is not clear. It seems that for a set of values of $c$ the non conservative estimator is slightly better than the conservative one. But reminding that in previous evaluation the errors of the two types of estimators were close, the results observed here seem consistent with previous ones.

Taking into account all that was seen and discussed in this section about the performance of the estimator, it can be concluded that the best choice is the non conservative approach. This will be considered in the next chapter (Chapter 4), where the estimator is used in real data sets. 


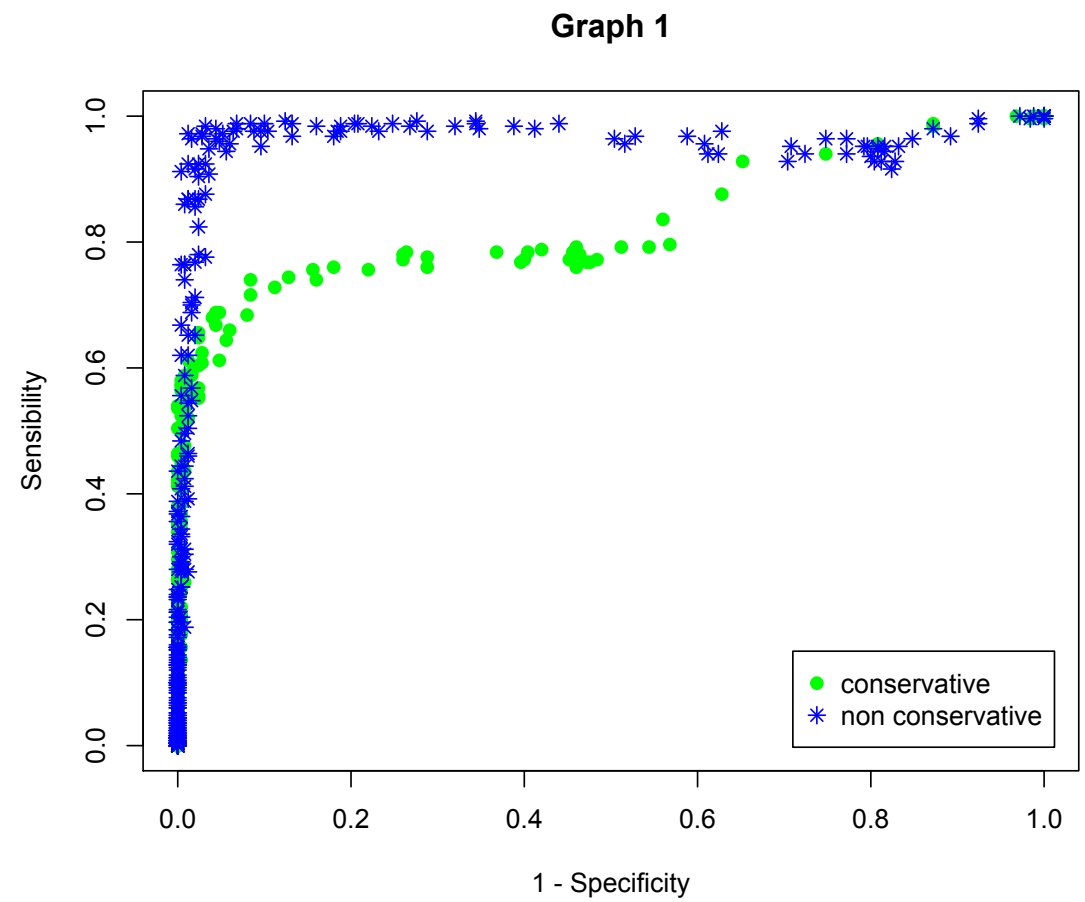

Figure 3.13: Graph of example 1: ROC curves for $\operatorname{cin}[0 ; 3.5]$ and $n=100$.

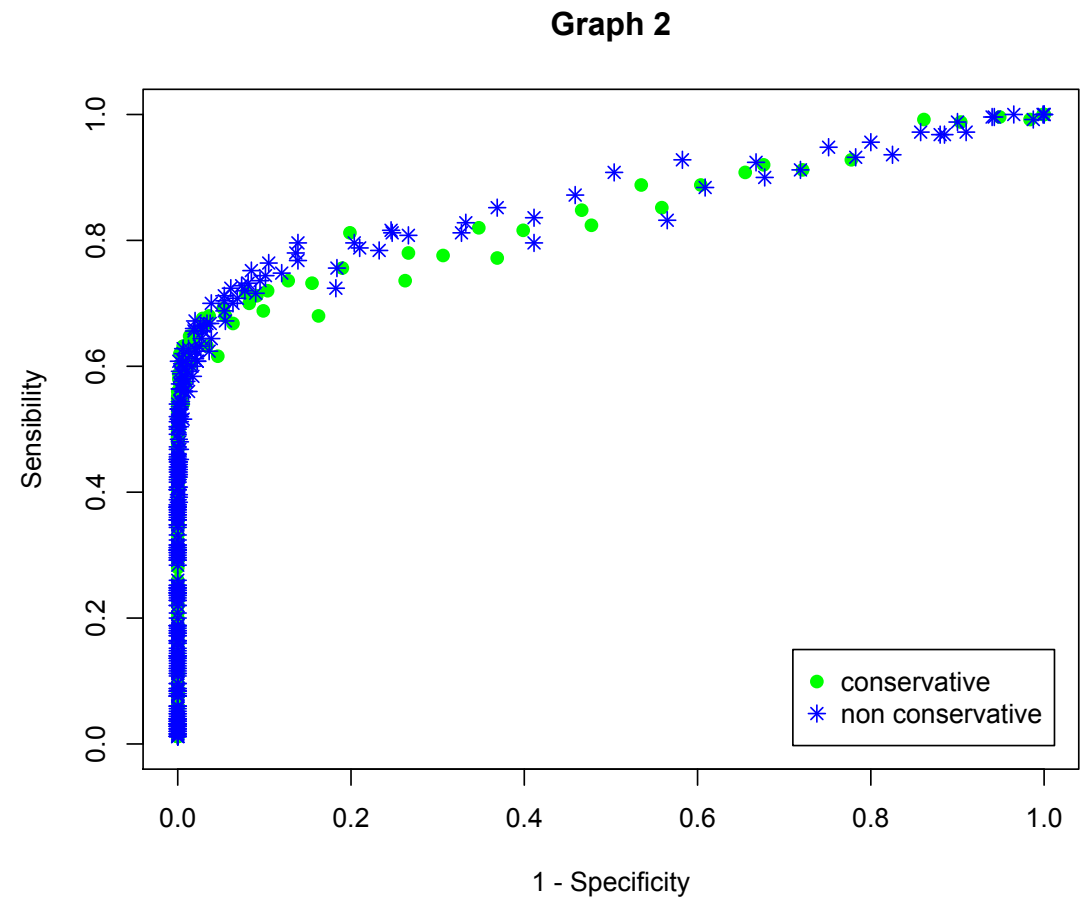

Figure 3.14: Graph of example 2: ROC curves for $\operatorname{cin}[0 ; 3.5]$ and $n=100$. 


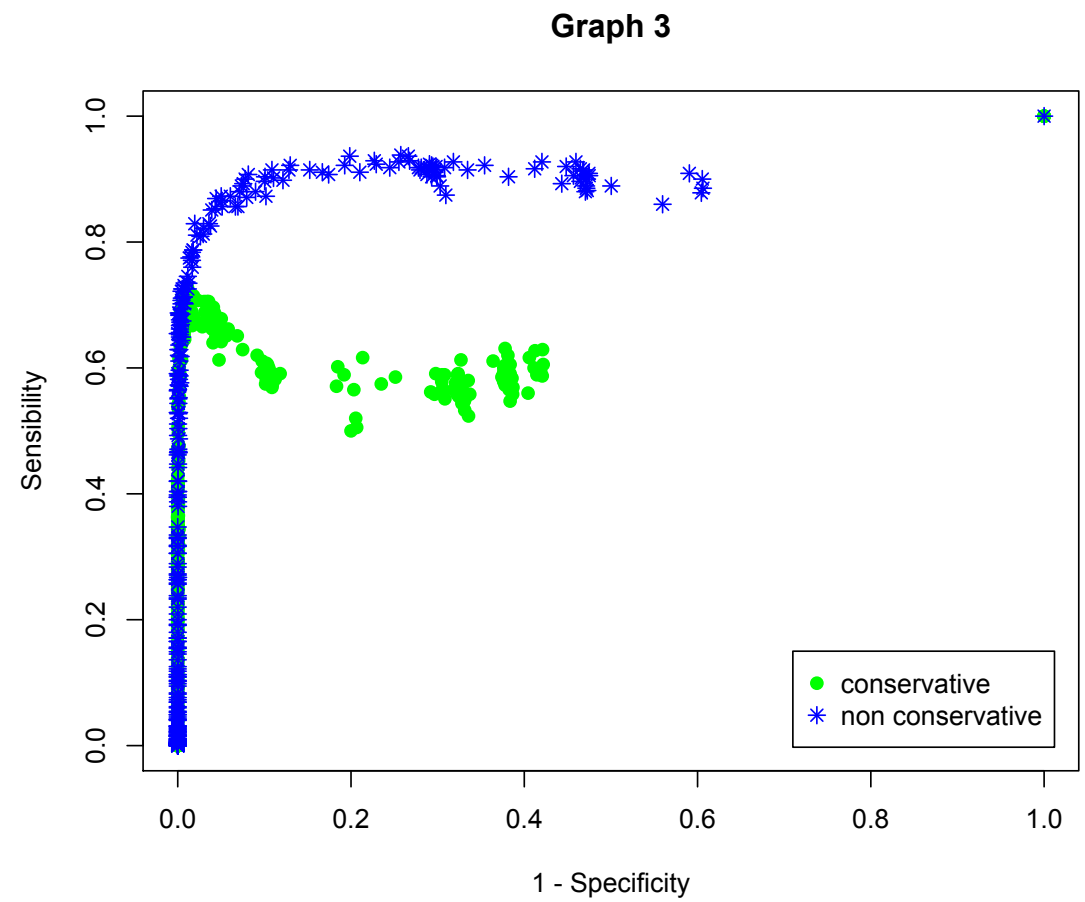

Figure 3.15: Graph of example 3: ROC curves for $\operatorname{cin}[0 ; 3.5]$ and $n=100$.

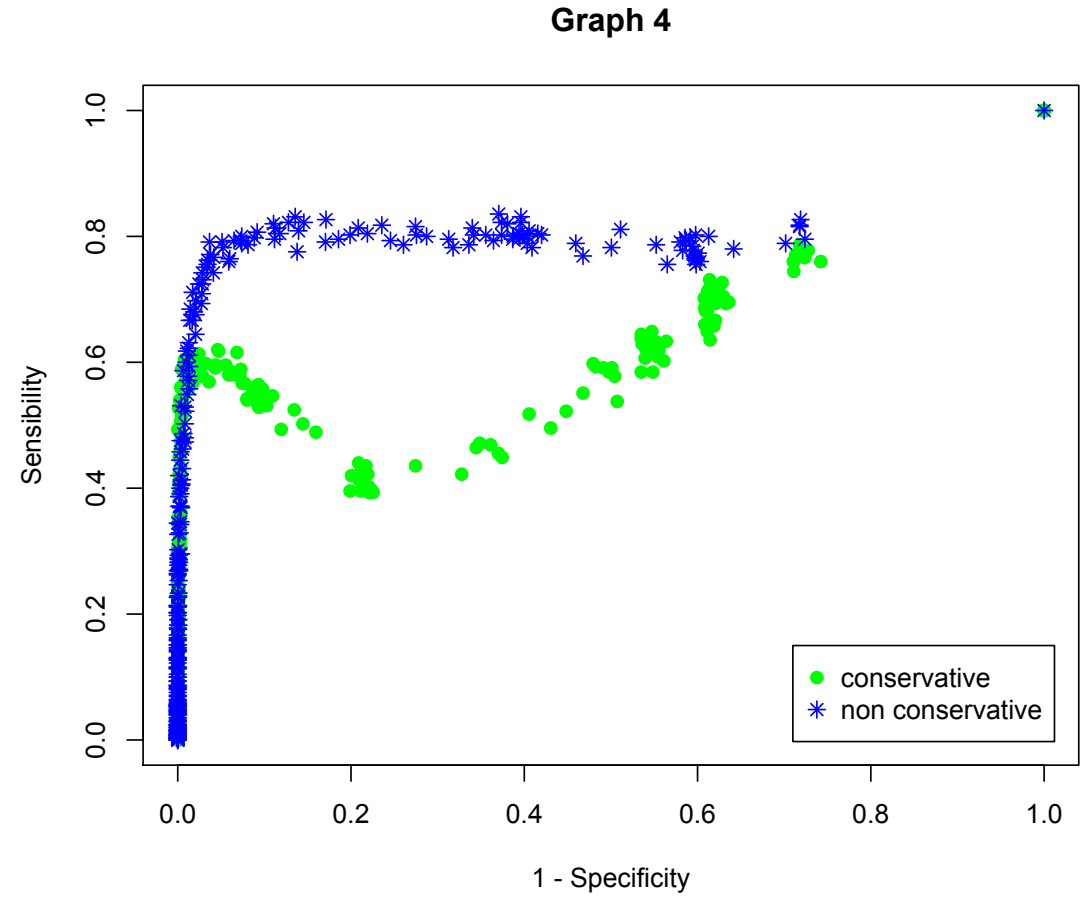

Figure 3.16: Graph of example 4: ROC curves for $\operatorname{cin}[0 ; 3.5]$ and $n=100$. 


\section{Chapter 4}

\section{Aplications}

This Chapter presents two aplications of random Markov fields in real data sets. The first one is an aplication in stock market indexes of five countries, that tries to understand the dependency relationships of the rise or fall of these indexes. The second aplication uses EEG (electroencephalogram) signals, captured by 20 electrodes during a visual experiment, and tries to find out the dependency relationships of these signals from different regions of the brain.

As already mentioned, we used here the results obtained in the previous chapter (Chapter 3), that is, we used the non conservative approach for the estimator. However it is necessary to choose a value for the constant $c$, and to solve the question of what is the best value for the constant $c$ we use a common resamplig method called cross-validation (see James et al. (2014) and Hastie et al. (2009)). The next section explain this method and how it is used in our context.

\subsection{Cross-validation}

There exists different approachs of this method, as the validation set approach, the leave-one-out cross-validation and the $k$-fold cross-validation. Here we use the $k$-fold cross-validation (James et al. (2014) and Hastie et al. (2009)). In this aproach the sample observations are randomly divided into $k$ groups, or folds, of approximately equal size. In summary one of the $k$ groups is treated as a validation set, and the remaining $k-1$ groups is the training set used to estimate the model. This procedure is repeated $k$ times; each time, a different group of observations is treated as a validation set and the measure of interest is calculated. So, at the end, this process results in $k$ estimates of this measure. And the $k$-fold cross-validation estimate is computed by averaging these values.

In linear models, for example, the measure of interest can be the mean squared error, but in our case we are interested in the log conditional likelihood of the model. So, for each value of $c$ this measure is calculated by the discribed method, and then the best $c$ is the one with the higher $\log$ conditional likelihood. Kepping in mind that here it is used the likelihood of the conditional distributions of each vertex $v \in V$ given its neighborhood, the log conditional likelihood of the model in the $i$-nth fold with constant $c$ is:

$$
\log \mathbb{P}(c)_{i}=\sum_{v \in V} \sum_{x_{\widehat{\mathrm{ne}}(v)} \in A^{\widehat{\mathrm{ne}}(v)}} \sum_{a \in A} N_{i}\left(a, x_{\widehat{\mathrm{ne}}(v)}\right) \log \hat{p}\left(a \mid x_{\widehat{\mathrm{ne}}(v)}\right),
$$

where the condinal probability $\hat{p}\left(a \mid x_{\widehat{\mathrm{ne}}(v)}\right)$ and the neighborhood $\widehat{\mathrm{ne}}(v)$ are obtained in the $k-1$ groups used to train the model, and the number $N_{i}\left(a, x_{\widehat{\mathrm{ne}}(v)}\right)$ comes from the $i$-nth group used to valitated the model (this can be calculated using the program in B.3). Then for each $c$, the measure that is used to compare and choose the best value of the constant is:

$$
C V(c)_{k}=\frac{1}{k} \sum_{i=1}^{k} \log \mathbb{P}(c)_{i}
$$


Once the value $c_{k}^{*}=\max _{c} C V(c)_{k}$ is found, the graph is estimated using the constant $c_{k}^{*}$ and the entire sample. For both aplications the samples were divided in $k=10$ folds.

\subsection{Stock market indexes}

In this aplication we took the stock market indexes of five countries, to know: Germany, Australia, Brazil, Spain and Japan. More than 14 years of data ${ }^{1}$ were collected, from April $18^{\text {th }}$ of 2001 to November $30^{\text {th }}$ of 2015 . And, excluding weekends and national holidays, the total number of observations is 3.218 days. Figure 4.1 shows the variation of those indexes over the 3.218 days, and it is hard to see any possible correlation between the indexes of the countries. However, we can use a graph to represent the conditional dependency relationships between the ups and downs of these indexes.

\section{Stock market indexes}

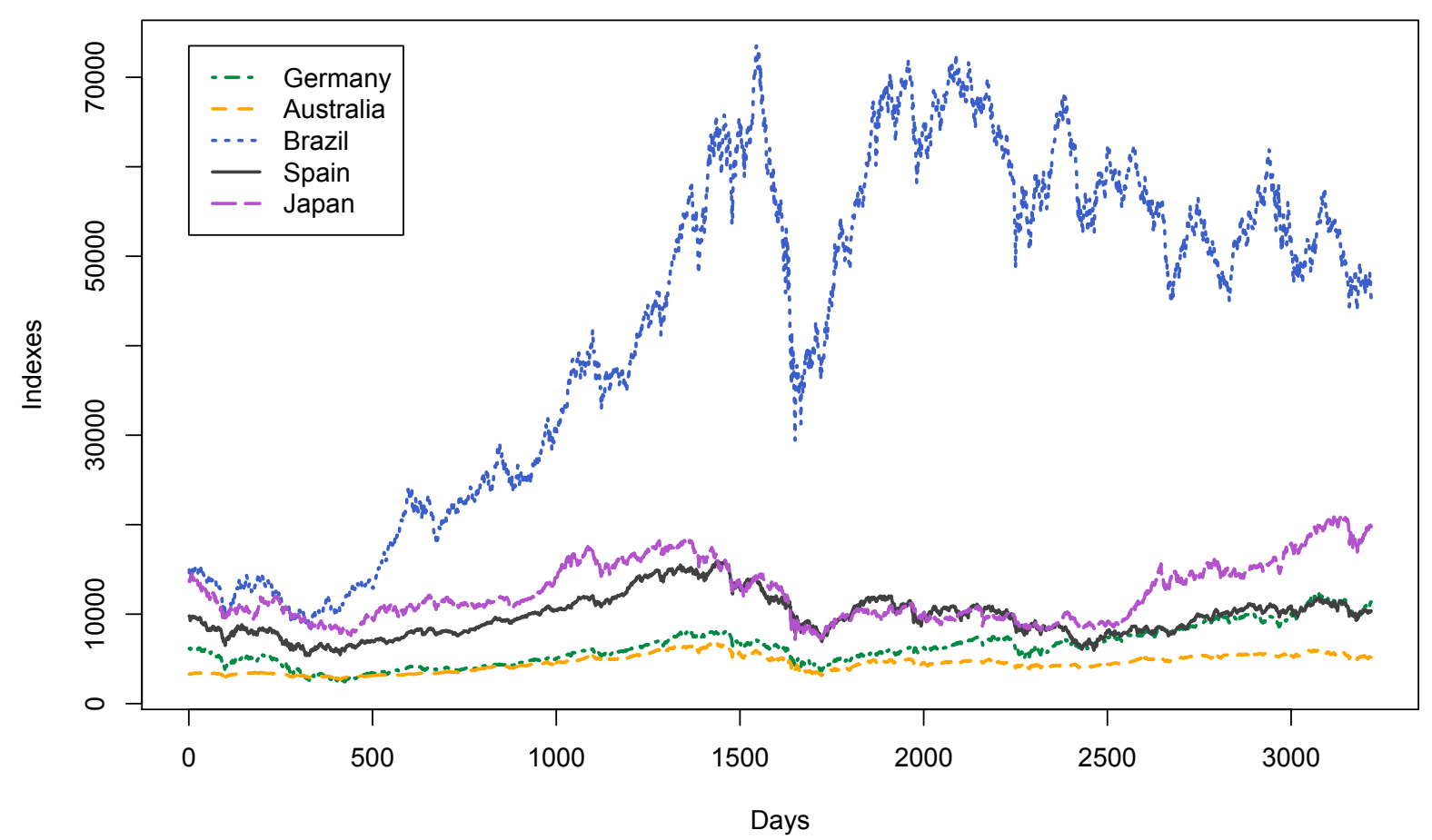

Figure 4.1: Stock market indexes from the five countries, from April $18^{\text {th }}$ of 2001 to November $30^{\text {th }}$ of 2015 .

So, to use the model proposed in this thesis we have to transform the data of a continuous set to a finite alphabet, and we did as follow: if the index of day $d+1$ is higher than the index of day $d$ the new observation take the value 1 , otherwise the new observation take the value 0 . Then we have the alphabet $A=\{0,1\}$ and 3.217 observations (one less than the total number of days, since we are evaluating the variation between days). One thing that we can not forget is that, even if we use this new observations that represents the variation between days, this observations can still not be independent, we will always have this kind of problems when dealing with time series data. To avoid this, some actions can be taken, as use only $10 \%$ of the observations, ensuring that the chosen observations have a gap of 9 observations between them. This gap can be found analyzing the autocorrelation of the observations, but even if this gap is less than 9 , for example 5 , we lose

\footnotetext{
${ }^{1}$ the data were obtained from this website http://br.investing.com/indices/major-indices
} 
information, and sometimes we cannot afford this. For this aplication, even with this problem in mind, we decided to use the entire sample.

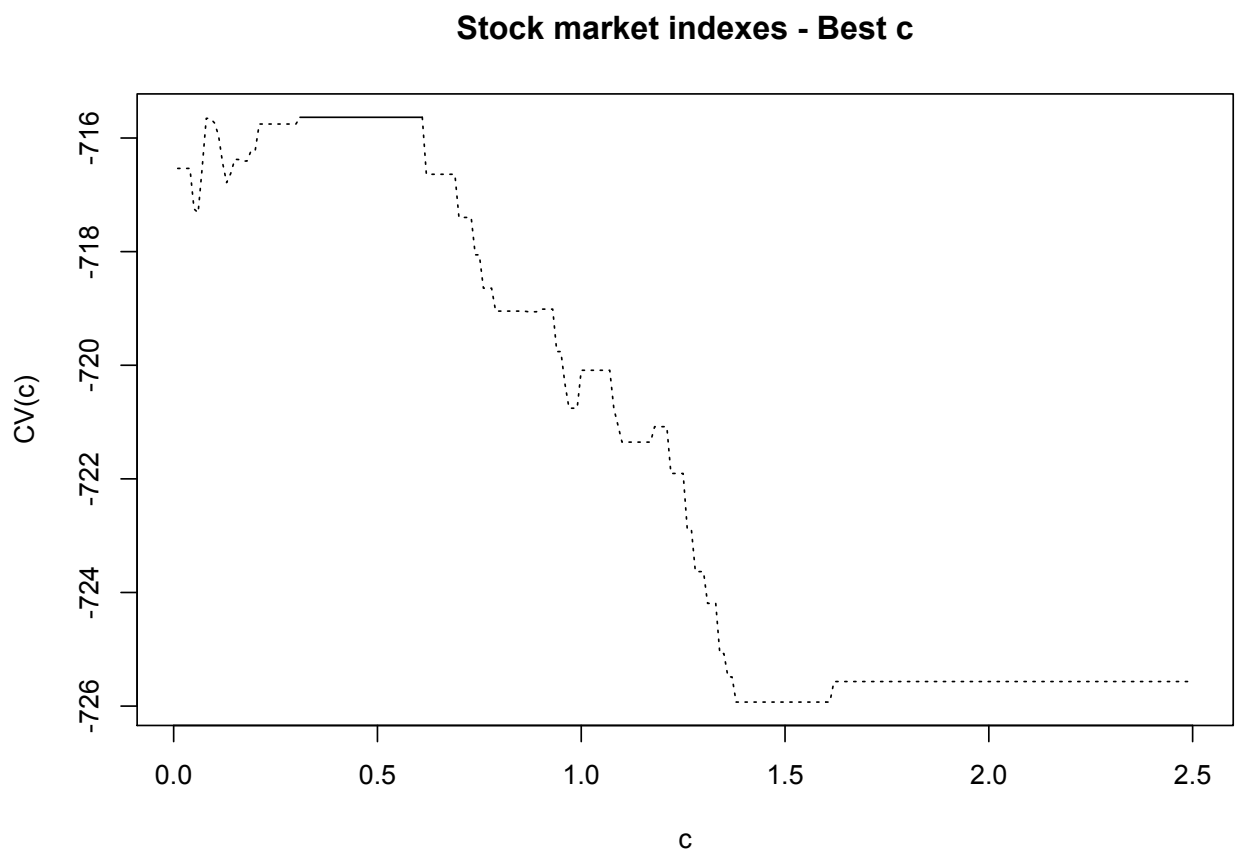

Figure 4.2: Stock market indexes: $C V(c)_{10}$, for $c=[0.01,2.5]$.

Using the concepts of $k$-fold cross-validation explained in the previous section, and taking $k=10$ as said, we performed the procedure to find the value $c^{*}$ assessing 250 values for $c$, from 0.01 to 2.5 , and Figure 4.2 shows the results. Note that the highest value obtained was $C V\left(c^{*}\right)_{10}=-715.6354$ for $c^{*}=[0.31,0.61]$ (the continuous line).

Stock market indexes - Graph

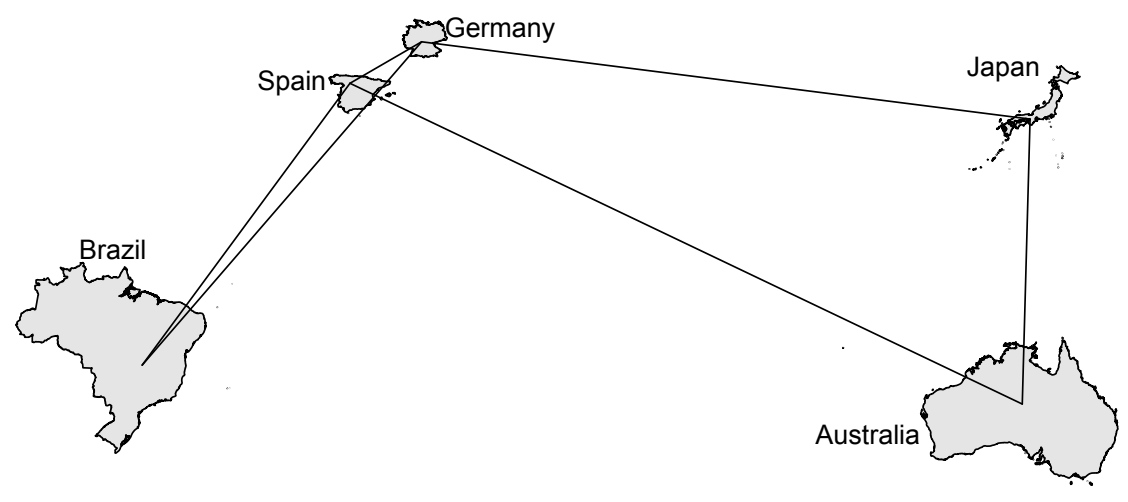

Figure 4.3: Stock market indexes: graph that represents the relationshio between the countries stock market indexes. 
Estimating the graph considering the values of $c^{*}=[0.31,0.61]$ and the whole sample we got the graph in Figure 4.3. This graph represents the relationship between the countries stock market indexes ups and downs. Note that Germany and Spain have three neighbors each, Germany has Brazil, Spain and Japan as its neighbors, and Spain has Brazil, Germany and Australia. And Brazil, Australia and Japan only have two neighbors each one, Brazil has Spain and Germany, Japan has Spain and Australia, and Australia has Spain and Japan. The result shows that the connections seem to be related to the geographical proximity of the countries.

\subsection{EEG signals}

An interesting application of the model is to study the relationships of dependence in neuroscience data. In this case, we study electroencephalograms (EEG) data, which are electric currents produced in the brain. The capture of these currents is performed through electrodes applied to the scalp, the brain surface, or even within the brain matter.

Here we use the data obtained by Prof. Claudia D. Vargas ${ }^{2}$ group. These data were collected by 20 electrodes placed on the scalp, which are positioned using the international 10-20 system. By this system, the nomenclature of the electrodes is given according to the region where they are located: $\mathrm{Fp}=$ frontal polar, $\mathrm{F}=$ frontal, $\mathrm{T}=$ temporal, $\mathrm{C}=$ central, $\mathrm{P}=$ parietal and $\mathrm{O}=$ occipital. The electrodes placed on the line that goes from nose to the nape are indexed by the letter $\mathrm{z}$ (from zero), those located on the left side of this line are indexed by odd number and on the right side by even numbers. Figure 4.4 shows the positions of the electrodes and the nomenclature used in this study.

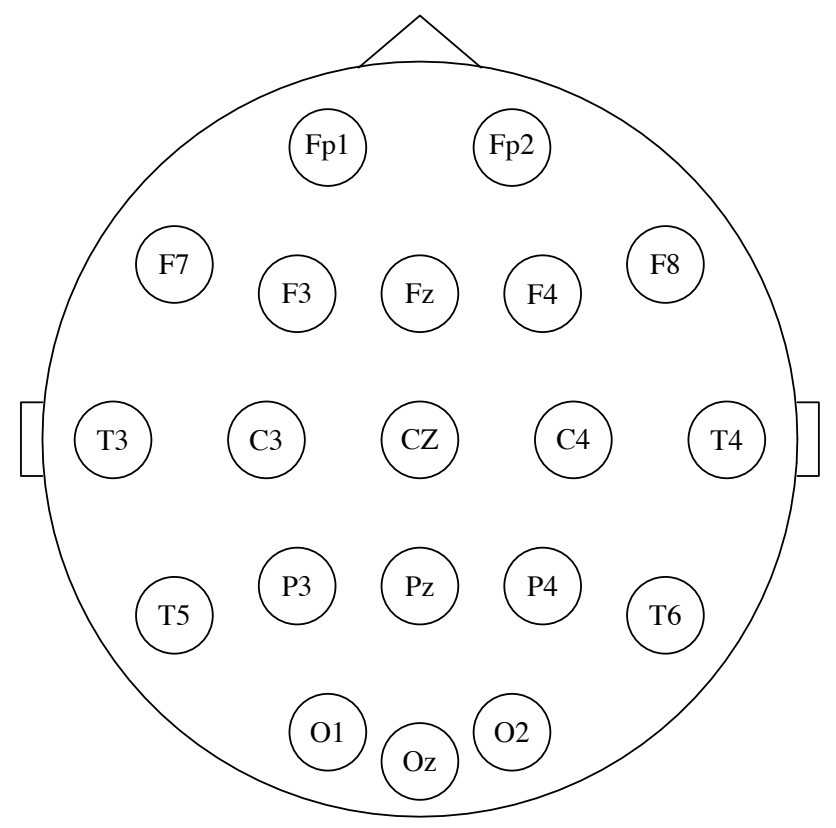

Figure 4.4: EEG signals: Position of electrodes on the scalp.

The data were collected during a visual experiment where the subject observes a short film in which a doll walks and then disappear, as it passed behind a wall. In these two moments, the walking and the occlusion, the EEG signals were captured. For the walking stage 1.000 points were recorded and for the occlusion stage 1.300 points were recorded in each one of the 20 electrodes. Altogether, 8 people participated in the experiment and each have different numbers of repetitions: $25,41,40,38,42,47,32$ and 39 (a total of 304 repetitions).

The first question we have to solve is the choice of how to discretize the signals, as these are continuous. It is known that this type of signal shows brain activity in its peaks and valleys, so the

\footnotetext{
${ }^{2}$ from Federal University of Rio de Janeiro
} 
choice of discretization took this information into account. Such a way that the peaks and valleys are marked with the value 1 , while the other points were marked with 0 . Then, to recognize these points the following procedure was done:

- for the signal of each combination stage (walking, occlusion), repetition $(1,2, \ldots, 304)$ and eletrode $(1,2, \ldots, 20)$, the first and third quartiles were calculated;

- if the point is less than the first quartil or if it is greater than the third quartil, the observation received the value 1 , otherwise the observation received the value 0 .

Doing that we transformed the continuos observations into an alphabet $A=\{0,1\}$.
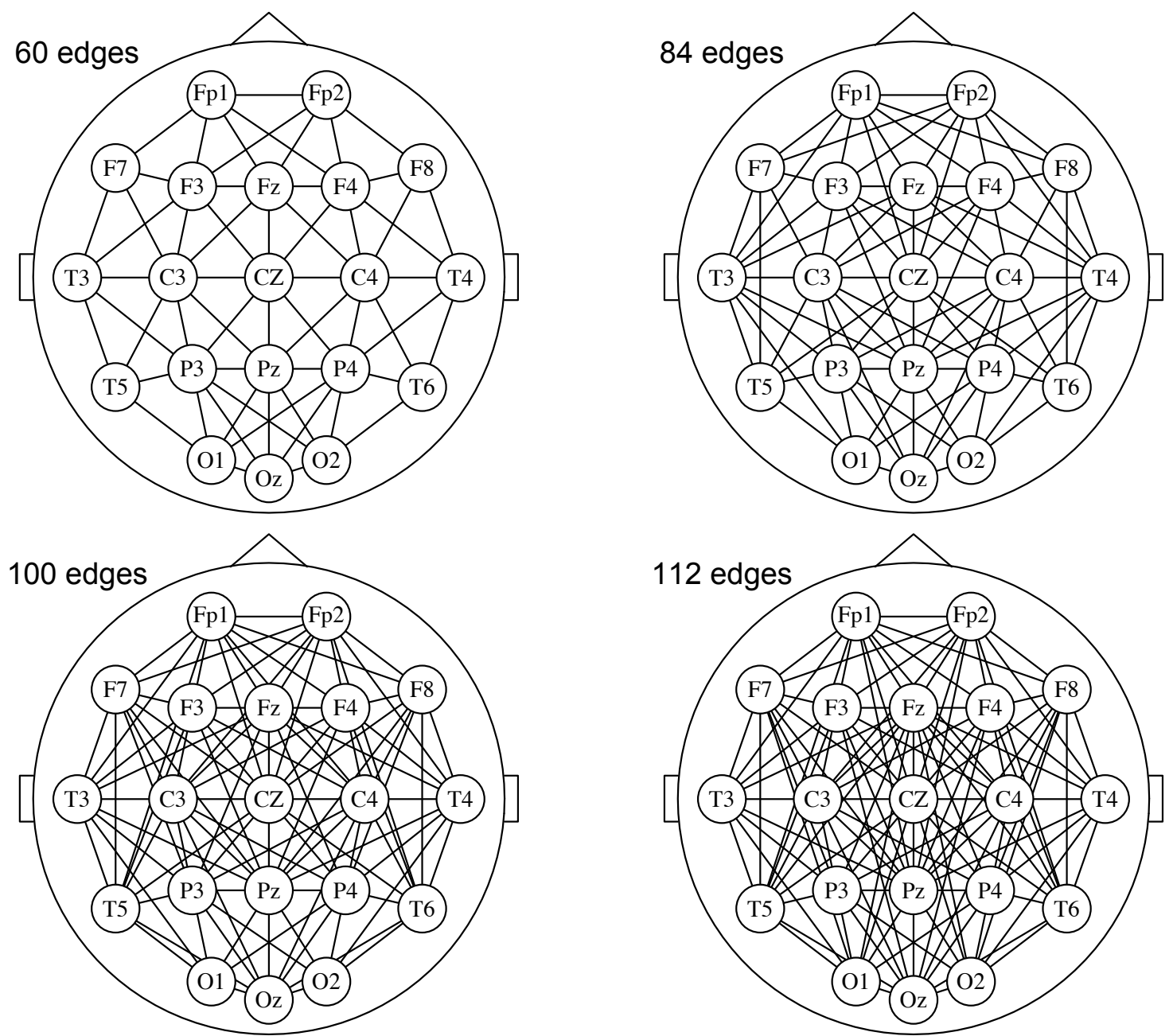

Figure 4.5: EEG signals: The four different graphs used to start the model estimation.

The second question to addrees is the lack of independence between observations. It is clear that within the sequence formed by these points there are no independence, and a preliminary assessment showed that the autocorrelation in these sequences are far from zero even for high lag values, such as 20 or 30. So to solve this problem we did the following: for each repetition of each stage we sellected from the original sample one observation every 100, ensuring a distance of 100 points between observations. Thereby, each repetition of the walking stage has size 10 and each repetition of the occlusion stage has size 13 . Then we put together the elements of each repetition of each person into a single sample for each stage, so the sample sizes are 3,040 for the walking stage and 3,952 for the occlusion stage. Because the goal is to find for each stage the graph that represents the interactions between the signals captured by the electrodes and identify whether there are differences between the graphs. 
As explain in Chapter 3, it is possible to choose a set of neighbors smaller than the set that represents the complet graph to be tested in the estimation program. Because of the large number of possible neighbors, 19 for each electrode, we decided to use the option of reduce the number of neighbors tested. We tested four different graphs to start the estimation, in each new test we increased the number of edges: starting with 60 edges, then 84, 100 and 112 edges (keep in mind that the complete graph has 190 edges). Figure 4.5 shows this four graphs.

Again we used the concept of $k$-fold cross-validation (with $k=10$ ) to find the value $c^{*}$ assesing 21 values, from 0.001 to 1 . For each one of the four different inicial graphs and stages (walking and occlusion) the results for $c^{*}$ was the same, we got $c^{*}=0.001$. Estimating the models again using the entire samples and $c=0.001$, the graphs estimated were equal to the graph used to start the estimation for both stages, no matter which graph was used to start the estimation.

We conclude that this model could not identify differences between the stages, if such differences exist. Note that other approaches to the treatment of samples or initiating the estimation by the complete graph could cause other results than those observed here. 


\section{Chapter 5}

\section{Generalization to Markov random fields with countable infinite set of vertices}

The theoretical results presented until now were just intended for discrete Markov random fields over $A^{V}$ with graph $G$, where $V$ is a finite set. In this chapter we generalize these results to the case of a countable infinite set of variables.

\subsection{Basic lemmas}

Note that Lemmas 3 and 4 of Chapter 2 can not be used and must be changed to fit the new condition imposed by $\mathrm{V}$ countable infinite. So, despite the definition of a Markov random field being the same, the proof of the existence of a minimum Markov neighborhood can not be achived using the finite intersection of all Markov neighborhoods, but it is possible to use a given Markov neighborhood to define it. That is, for $v \in V$, let $W_{v}$ be a given Markov neighborhood of $v$ (that it is assumed to exist) and define

$$
\Theta(v)=\left\{W \subset W_{v}: W \text { is a Markov neighborhood of } v\right\}
$$

Now, define as in Chapter 2,

$$
\operatorname{ne}(v)=\bigcap_{W \in \Theta(v)} W
$$

Lemma 10. For all $v \in V$, ne $(v)$ defined by (5.1) is a Markov neighborhood of $v$ and ne $(v) \subset \Phi$ for all $\Phi \subset V$ Markov neighborhood of $v$.

Proof. Because ne $(v)$ is a finite intersection of Markov neighborhoods, by the Markov intersection property, ne $(v)$ is a Markov neighborhood as it is the intersection of any Markov neighborhood $\Phi$ and $W_{v}$.

So, because $\Phi \cap W_{v}$ is a Markov neighborhood and $\Phi \cap W_{v} \subset W_{v}$, then ne $(v) \subset \Phi \cap W_{v}$. On the other hand, $\Phi \cap W_{v} \subset \Phi$ wich implies that ne $(v) \subset \Phi$, for all $\Phi \subset V$ Markov neighborhood of $v$.

Recalling that the conditional distribution of $X_{v}$ given $X_{\mathrm{ne}(v)}=a_{\mathrm{ne}(v)}$ is denoted by $\left\{p\left(a \mid a_{\mathrm{ne}(v)}\right)\right\}_{a \in A}$; i.e., for all $a \in A$ we have

$$
p\left(a \mid a_{\mathrm{ne}(v)}\right)=\mathbb{P}\left(X_{v}=a \mid X_{k}=a_{k}, k \in \operatorname{ne}(v)\right) .
$$

And similarly, the joint distribution of $\left(X_{v}, X_{\mathrm{ne}(v)}\right)$ is denoted by $\left\{p\left(a_{v}, a_{\mathrm{ne}(v)}\right)\right\}_{a_{v} \in A, a_{\mathrm{ne}(v)} \in A^{\mathrm{ne}(v)}}$. Here we present the generalization of Lemma 4 for the case of $V$ countable infinite, as presented in Lemma A.2 of Csiszár and Talata (2006). 
Lemma 11. For a Markov random field over $A^{V}$, let ne $(v)$ be the smallest Markov neighborhood of $v \in V$. If a neighborhood $W$ satisfies

$$
p\left(a \mid a_{W}\right)=p\left(a \mid a_{n e(v)}\right)
$$

then $W$ is a Markov neighborhood.

Proof. We have to show that for any $\Delta \supset W$ finite with $\Delta \subset V$,

$$
p\left(a \mid x_{\Delta}\right)=p\left(a \mid x_{W}\right) .
$$

As ne $(v)$ is a Markov neighborhood, the lemma's condition implies

$$
p\left(a \mid a_{W}\right)=p\left(a \mid a_{\mathrm{ne}(v)}\right)=p\left(a \mid a_{\mathrm{ne}(v) \cup \Delta}\right) .
$$

So (5.3) follows, because $W \subseteq \Delta \subseteq$ ne $(v) \cup \Delta$.

The following section presents all the arguments, assumptions and definitions needed to handle the nuances of working with an countable infinite graph.

\subsection{Model selection when $V$ is countable infinite}

Previously, a sample with size $n$ of $\left\{X_{v}: v \in V\right\}$ meant, in simple words, a matrix of dimensions $n \times|V|$. Now for $i=1, \ldots, n$ we assume the observation of an independent sample of $\left\{X_{v}: v \in V_{n}\right\}$, where $V_{n}$ is a finite subset of $V, V_{n} \uparrow V$ when $n \rightarrow \infty$, i.e. $V_{1} \subseteq V_{2} \subseteq V_{3} \subseteq \ldots$.

The estimation of the conditional probabilities for the sample stays the same, because we focus on finite subsets of $V$. The diferences happen in the estimation of the Markov neighborhoods and by consequence in the reconstruction of the graph. Actually now we just can reconstruct a finite subgraph of $G$, with a finite sample, because $G$ has a countable infinite number of nodes.

Definition 12. Given a function $c=c(n)>0$, the empirical neighborhood of the vertex $v$ is the set of indices $\widehat{n e}(v)$ given by

$$
\widehat{n e}(v)=\underset{W \subset V_{n} \backslash\{v\}}{\arg \max }\left\{\log \hat{\mathbb{P}}_{v \mid W}\left(x_{v}^{(1: n)} \mid x_{W}^{(1: n)}\right)-c|A|^{|W|} \log _{|A|} n\right\} .
$$

Definition 13. Define $p_{\min }(v)$ as

$$
p_{\min }(v)=\inf \left\{p\left(a \mid a_{W}\right): p\left(a \mid a_{W}\right)>0, a \in A, a_{W} \in A^{W}, W \subset V \text { finite and } v \notin W\right\} .
$$

Now consider the following assumptions:

Assumption 14. $\inf _{v \in V}\left\{p_{\min }(v)\right\}=p_{*}>0$.

Assumption 15. The sequence $\left\{V_{n}: n \geq 1\right\}$ satisfies $\left|V_{n}\right| \leq o(\log n)$.

Assumption 16. For all $n \in \mathbb{N}, c>\frac{32|A|}{p_{*}^{\left|V_{n}\right|+1}}$.

Assumption 17. For all $v \in V$,

$$
\inf _{W \not \subset n e(v), n e(v) \not \subset W} D\left(p\left(\cdot \mid a_{n e(v)}\right) ; p\left(\cdot \mid a_{W}\right)\right) \geq \alpha>0 .
$$

We prove the following consistency result for the neighborhood estimator.

Theorem 18. Under the Assumptions 14, 15, 16 and 17, the estimator given by (5.4) satisfies $\widehat{n e}(v)=n e(v)$ eventually almost surely as $n \rightarrow \infty$. 
Here we are interested in estimating a finite subgraph of $G$. We still can estimate the neighborhood of each node and reconstruct the subgraph based on the set of neighborhoods. Given a set $V^{\prime} \subset V$, we denote by $G_{V^{\prime}}$ the induced subgraph; that is, the graph given by the pair $\left(V^{\prime}, E^{\prime}\right)$, where $E^{\prime}=\left\{(v, w) \in E: v, w \in V^{\prime}\right\}$. Based on the neighborhood estimator (5.4), we can construct an estimator of the subgraph $G_{V^{\prime}}$ for any $V^{\prime} \subset V$ finite, by defining the set of edges

$$
\widehat{E}_{V^{\prime}}^{\wedge}=\left\{(v, w) \in V^{\prime} \times V^{\prime}: v \in \widehat{\mathrm{ne}}(w) \text { and } w \in \widehat{\mathrm{ne}}(v)\right\} .
$$

The estimated subgraph will be the pair $\widehat{G}_{V^{\prime}}^{\wedge}=\left(V^{\prime}, \widehat{E}_{V^{\prime}}^{\wedge}\right)$. In the same way, if we want to be less conservative we can define

$$
\widehat{E}_{V^{\prime}}^{\vee}=\left\{(v, w) \in V^{\prime} \times V^{\prime}: v \in \widehat{\operatorname{ne}}(w) \text { or } w \in \widehat{\operatorname{ne}}(v)\right\}
$$

and the estimated subgraph will be the pair $\widehat{G}_{V^{\prime}}^{\vee}=\left(V^{\prime}, \widehat{E}_{V^{\prime}}^{\vee}\right)$.

Corollary 19. For any finite set $V^{\prime} \subset V$ we have $\widehat{G}_{V^{\prime}}^{\wedge}=\widehat{G}_{V^{\prime}}^{\vee}=G_{V^{\prime}}$ eventually almost surely as $n \rightarrow \infty$.

Now we prove the following theorem inspired in Theorema 1b) of Csiszár (2002) and we derived a corollary.

Theorem 20. Let $V_{n} \subset V$, with $\left|V_{n}\right| \leq o(\log n)$. Then for all $\delta>2$ eventually almost surely as $n \rightarrow \infty$

$$
\left|\frac{\hat{p}\left(a_{W}\right)}{p\left(a_{W}\right)}-1\right| \leq \sqrt{\frac{\delta \log n}{n p\left(a_{W}\right)^{2}}}
$$

simultaneously for all $W \subset V_{n}$ and $a_{W} \in A^{W}$.

Proof. For $a_{W} \in A^{W}$, let

$$
Y_{i}=\mathbf{1}\left\{x_{W}^{(i)}=\left(a_{W}\right)\right\}-p\left(a_{W}\right) i=1,2, \ldots, n .
$$

Note that $\mathbb{E}\left(Y_{i}\right)=0$ and $Y_{i} \in[-1,1], \forall i=1,2, \ldots, n$, then by Hoeffding's Inequality (Appendix A, Theorem 28)

$$
\mathbb{P}\left(\left|\frac{1}{n} \sum_{i=1}^{n} Y_{i}-\mathbb{E}\left[\frac{1}{n} \sum_{i=1}^{n} Y_{i}\right]\right| \geq t\right) \leq 2 \exp \left(-\frac{2 n^{2} t^{2}}{\sum_{i=1}^{n}(1-(-1))^{2}}\right) .
$$

We have that

$$
\frac{1}{n} \sum_{i=1}^{n} Y_{i}=\frac{N\left(a_{W}\right)}{n}-p\left(a_{W}\right)
$$

and because $\mathbb{E}\left(Y_{i}\right)<\infty$,

$$
\mathbb{E}\left[\frac{1}{n} \sum_{i=1}^{n} Y_{i}\right]=\frac{1}{n} \sum_{i=1}^{n} \mathbb{E}\left(Y_{i}\right)=\frac{1}{n} \sum_{i=1}^{n} 0=0
$$

Therefore,

$$
\begin{aligned}
& \mathbb{P}\left(\left|\frac{N\left(a_{W}\right)}{n}-p\left(a_{W}\right)\right| \geq t\right) \leq 2 \exp \left(-\frac{2 n^{2} t^{2}}{n 4}\right) \\
& =2 \exp \left(-\frac{n t^{2}}{2}\right)
\end{aligned}
$$


Take $t=\sqrt{\frac{\delta \log n}{n}}$, then

$$
\begin{aligned}
& \mathbb{P}\left(\left|\frac{\hat{p}\left(a_{W}\right)}{p\left(a_{W}\right)}-1\right| \geq \frac{t}{p\left(a_{W}\right)}\right) \\
& =\mathbb{P}\left(\left|\frac{\hat{p}\left(a_{W}\right)}{p\left(a_{W}\right)}-1\right| \geq \sqrt{\frac{\delta \log n}{n p\left(a_{W}\right)^{2}}}\right) \leq 2 \exp \left(-\frac{\delta \log n}{2}\right)
\end{aligned}
$$

Thus, we obtain

$$
\begin{aligned}
& \mathbb{P}\left\{\left|\frac{\hat{p}\left(a_{W}\right)}{p\left(a_{W}\right)}-1\right| \geq \sqrt{\frac{\delta \log n}{n p\left(a_{W}\right)^{2}}} \text { for some } W \subset V_{n} \text { and } a_{W} \in A^{W}\right\} \\
& \leq \sum_{W \subset V_{n}} \sum_{a_{W} \in A^{W}} \mathbb{P}\left\{\left|\frac{\hat{p}\left(a_{W}\right)}{p\left(a_{W}\right)}-1\right| \geq \sqrt{\frac{\delta \log n}{n p\left(a_{W}\right)^{2}}}\right\} \\
& \leq 2^{\left|V_{n}\right|}|A|^{\left|V_{n}\right|} 2 \exp \left(-\frac{\delta \log n}{2}\right) \\
& \leq 2^{o(\log n)}|A|^{o(\log n)} 2 \exp \left(-\frac{\delta \log n}{2}\right)
\end{aligned}
$$

which is summable in $n$ for $\delta>2$. This completes the proof.

Corollary 21. Suppose Assumptions 14 and 15 hold. Then for all $\delta>16$, eventually almost surely as $n \rightarrow \infty$ we have

$$
\left|\hat{p}\left(a \mid a_{W}\right)-p\left(a \mid a_{W}\right)\right| \leq \sqrt{\frac{\delta \log n}{p_{*}^{|W|+2} N\left(a_{W}\right)}}
$$

simultaneously for all $v \in V, W \subset V_{n} \backslash\{v\}, a \in A$ and $a_{W} \in A^{W}$.

Proof. Observe that

$$
\left|\hat{p}\left(a \mid a_{W}\right)-p\left(a \mid a_{W}\right)\right|=\left|\frac{N\left(a, a_{W}\right)}{N\left(a_{W}\right)}-\frac{p\left(a, a_{W}\right)}{p\left(a_{W}\right)}\right| .
$$

By summing and subtracting $N\left(a, a_{W}\right) / n p\left(a_{W}\right)$ we obtain

$$
\begin{aligned}
\left|\hat{p}\left(a \mid a_{W}\right)-p\left(a \mid a_{W}\right)\right| & \leq\left|\frac{N\left(a, a_{W}\right)}{N\left(a_{W}\right)}-\frac{N\left(a, a_{W}\right)}{n p\left(a_{W}\right)}\right|+\left|\frac{N\left(a, a_{W}\right)}{n p\left(a_{W}\right)}-\frac{p\left(a, a_{W}\right)}{p\left(a_{W}\right)}\right| \\
& =\frac{N\left(a, a_{W}\right)}{N\left(a_{W}\right)}\left|1-\frac{N\left(a_{W}\right)}{n p\left(a_{W}\right)}\right|+p\left(a \mid a_{W}\right)\left|\frac{N\left(a, a_{W}\right)}{n p\left(a, a_{W}\right)}-1\right| \\
& \leq\left|1-\frac{N\left(a_{W}\right)}{n p\left(a_{W}\right)}\right|+\left|\frac{N\left(a, a_{W}\right)}{n p\left(a, a_{W}\right)}-1\right| \\
& =\left|1-\frac{\hat{p}\left(a_{W}\right)}{p\left(a_{W}\right)}\right|+\left|\frac{\hat{p}\left(a, a_{W}\right)}{p\left(a, a_{W}\right)}-1\right|
\end{aligned}
$$

Therefore, by Theorem 20 we have that for all $\delta>2$, eventually almost surely as $n \rightarrow \infty$ we have

$$
\begin{aligned}
\left|\hat{p}\left(a \mid a_{W}\right)-p\left(a \mid a_{W}\right)\right| & \leq \sqrt{\frac{\delta \log n}{n p\left(a_{W}\right)^{2}}}+\sqrt{\frac{\delta \log n}{n p\left(a, a_{W}\right)^{2}}} \\
& \leq \sqrt{\frac{4 \delta \log n}{n p\left(a, a_{W}\right)^{2}}}
\end{aligned}
$$


simultaneously for all $v \in V, W \subset V_{n} \backslash\{v\}, a \in A$ and $a_{W} \in A^{W}$. But Theorem 20 and Assumption 14 also implies that eventually almost surely as $n \rightarrow \infty$,

$$
n p\left(a, a_{W}\right)=n p\left(a \mid a_{W}\right) p\left(a_{W}\right) \geq p\left(a \mid a_{W}\right) N\left(a_{W}\right) / 2
$$

for all $v \in V, W \subset V_{n} \backslash\{v\}, a \in A$ and $a_{W} \in A^{W}$. Then, as $p\left(a \mid a_{W}\right) p\left(a, a_{W}\right) \geq p_{*}^{|W|+2}$ we obtain

$$
\begin{aligned}
\left|\hat{p}\left(a \mid a_{W}\right)-p\left(a \mid a_{W}\right)\right| & \leq \sqrt{\frac{8 \delta \log n}{p\left(a \mid a_{W}\right) p\left(a, a_{W}\right) N\left(a_{W}\right)}} \\
& \leq \sqrt{\frac{8 \delta \log n}{p_{*}^{|W|+2} N\left(a_{W}\right)}}
\end{aligned}
$$

and the statement of the corollary follows.

Proof of Theorem 18. Denote by

$$
\operatorname{PML}\left(x_{v}^{(1: n)} \mid x_{W}^{(1: n)}\right)=\log \hat{\mathbb{P}}_{v \mid W}\left(x_{v}^{(1: n)} \mid x_{W}^{(1: n)}\right)-c|A|^{|W|} \log n .
$$

where

$$
\hat{\mathbb{P}}\left(x_{v}^{(1: n)} \mid x_{W}^{(1: n)}\right)=\prod_{a_{W} \in A^{W}} \prod_{a \in A} \hat{p}\left(a \mid a_{W}\right)^{N\left(a, a_{W}\right)} .
$$

If $V_{n} \backslash\{v\}$ is the bounding set for the neighborhood of vertex $v$ and ne $(v)$ is the minimal neighborhood of $v$, we have to prove that

$$
\max _{W \subset V_{n} \backslash\{v\}, W \neq \operatorname{ne}(v)} \operatorname{PML}\left(x_{v}^{(1: n)} \mid x_{W}^{(1: n)}\right)<\operatorname{PML}\left(x_{v}^{(1: n)} \mid x_{\mathrm{ne}(v)}^{(1: n)}\right)
$$

eventually almost surely $n \rightarrow \infty$. And again, we divide the proof in three cases, showing that

$$
\max _{W \in \mathcal{B}_{i}} \operatorname{PML}\left(x_{v}^{(1: n)} \mid x_{W}^{(1: n)}\right)<\operatorname{PML}\left(x_{v}^{(1: n)} \mid x_{\mathrm{ne}(v)}^{(1: n)}\right)
$$

where

(a) $\mathcal{B}_{1}=\left\{W \subset V_{n} \backslash\{v\}: \operatorname{ne}(v) \subset W\right\}$

(b) $\mathcal{B}_{2}=\left\{W \subset V_{n} \backslash\{v\}: W \subset \operatorname{ne}(v)\right\}$

(c) $\mathcal{B}_{3}=\left\{W \subset V_{n} \backslash\{v\}: W \not \subset \operatorname{ne}(v)\right.$, ne $\left.(v) \not \subset W\right\}$.

Compared to the proof of Theorem 7, the biggest changes in this proof are in cases (a) and (c), where the cardinality of the sets $\mathcal{B}$ is unbounded. In case (b) the only changes are the substitutions of Lemmas 3 and 4 by Lemmas 10 and 11, respectively. Next is the proof of each case:

(a). We have to prove that

$$
\max _{W \in \mathcal{B}_{1}} \operatorname{PML}\left(x_{v}^{(1: n)} \mid x_{W}^{(1: n)}\right)<\operatorname{PML}\left(x_{v}^{(1: n)} \mid x_{\mathrm{ne}(v)}^{(1: n)}\right)
$$

eventually almost surely $n \rightarrow \infty$.

Observe that for all $W \in \mathcal{B}_{1}$

$$
\begin{aligned}
& \operatorname{PML}\left(x_{v}^{(1: n)} \mid x_{\mathrm{ne}(v)}^{(1: n)}\right)-\operatorname{PML}\left(x_{v}^{(1: n)} \mid x_{W}^{(1: n)}\right)= \\
& c\left(|A|^{|W|}-|A|^{|\operatorname{ne}(v)|}\right) \log n-\sum_{a, a_{W} \in A^{|W|+1}} N\left(a, a_{W}\right) \log \frac{\hat{p}\left(a \mid a_{W}\right)}{\hat{p}\left(a \mid a_{\mathrm{ne}(v)}\right)} .
\end{aligned}
$$


As these empirical probabilities are the maximum likelihood estimators we have that

$$
\begin{aligned}
\sum_{a, a_{W} \in A^{W+1}} N\left(a, a_{W}\right) \log \hat{p}\left(a \mid a_{\mathrm{ne}(v)}\right) & \geq \sum_{a, a_{W} \in A^{W+1}} N\left(a, a_{W}\right) \log p\left(a \mid a_{\mathrm{ne}(v)}\right) \\
& =\sum_{a, a_{W} \in A^{W+1}} N\left(a, a_{W}\right) \log p\left(a \mid a_{W}\right) .
\end{aligned}
$$

Therefore, (5.6) can be lower-bouded by

$$
c\left(1-\frac{1}{|A|}\right)|A|^{|W|} \log n-\sum_{a, a_{W} \in A^{W+1}} N\left(a, a_{W}\right) \log \frac{\hat{p}\left(a \mid a_{W}\right)}{p\left(a \mid a_{W}\right)} .
$$

Now, observe that

$$
\sum_{a, a_{W} \in A^{W+1}} N\left(a, a_{W}\right) \log \frac{\hat{p}\left(a \mid a_{W}\right)}{p\left(a \mid a_{W}\right)}=\sum_{a_{W} \in A^{W}} N\left(a_{W}\right) D\left(\hat{p}\left(\cdot \mid a_{W}\right) ; p\left(\cdot \mid a_{W}\right)\right),
$$

where $D$ denotes the Küllback-Leibler divergence (see Definition 22 in Appendix A). Therefore we have, by Lemma 24 (in Appendix A)

$$
\begin{aligned}
\sum_{a_{W} \in A^{W}} N\left(a_{W}\right) & D\left(\hat{p}\left(\cdot \mid a_{W}\right) ; p\left(\cdot \mid a_{W}\right)\right) \\
& \leq \sum_{a_{W} \in A^{W}} N\left(a_{W}\right) \sum_{a \in A} \frac{\left[\hat{p}\left(a \mid a_{W}\right)-p\left(a \mid a_{W}\right)\right]^{2}}{p\left(a \mid a_{W}\right)} .
\end{aligned}
$$

Then, by Corollary 21 we have for any $\delta>16$, that

$$
\begin{aligned}
\sum_{a_{W} \in A^{W}} N\left(a_{W}\right) & D\left(\hat{p}\left(\cdot \mid a_{W}\right) ; p\left(\cdot \mid a_{W}\right)\right) \\
& \leq \sum_{a_{W} \in A^{W}} N\left(a_{W}\right) \sum_{a \in A} \frac{\delta \log n}{N\left(a_{W}\right) p_{*}^{|W|+2}} \\
& =\sum_{a, a_{W} \in A^{W+1}} \frac{\delta \log n}{p_{*}^{|W|+2}} \\
& \leq \frac{\delta|A|^{|W|+1} \log n}{p_{*}^{|W|+2}}
\end{aligned}
$$

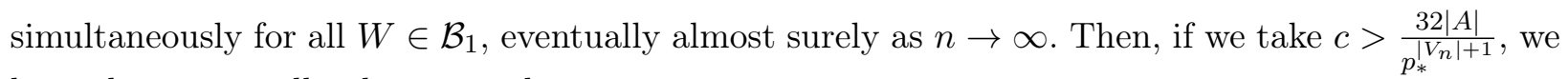
have that eventually almost surely as $n \rightarrow \infty$

$$
\max _{W \in \mathcal{B}_{1}} \operatorname{PML}\left(x_{v}^{(1: n)} \mid x_{W}^{(1: n)}\right)<\operatorname{PML}\left(x_{v}^{(1: n)} \mid x_{\mathrm{ne}(v)}^{(1: n)}\right) .
$$

This completes the proof of part (a).

(b) We have to prove that

$$
\max _{W \in \mathcal{B}_{2}} \operatorname{PML}\left(x_{v}^{(1: n)} \mid x_{W}^{(1: n)}\right)<\operatorname{PML}\left(x_{v}^{(1: n)} \mid x_{\operatorname{ne}(v)}^{(1: n)}\right)
$$

eventually almost surely as $n \rightarrow \infty$. 
In this case we have that for all $W \in \mathcal{B}_{2}$

$$
\begin{aligned}
\operatorname{PML}\left(x_{v}^{(1: n)} \mid x_{\mathrm{ne}(v)}^{(1: n)}\right)-\operatorname{PML}\left(x_{v}^{(1: n)} \mid x_{W}^{(1: n)}\right)= & \sum_{\left(a, a_{\mathrm{ne}(v)}\right) \in A^{\mathrm{ne}(v)+1}} N\left(a, a_{\mathrm{ne}(v)}\right) \log \frac{\hat{p}\left(a \mid a_{\mathrm{ne}(v)}\right)}{\hat{p}\left(a \mid a_{W}\right)}-c\left(|A|^{|\mathrm{ne}(v)|}-|A|^{|W|}\right) \log n \\
= & n\left[\sum_{\left(a, a_{\mathrm{ne}(v)}\right) \in A^{\mathrm{ne}(v)+1}} \frac{N\left(a, a_{\mathrm{ne}(v)}\right)}{n} \log \frac{\hat{p}\left(a \mid a_{\mathrm{ne}(v)}\right)}{\hat{p}\left(a \mid a_{W}\right)}-c\left(|A|^{|\operatorname{ne}(v)|}-|A|^{|W|}\right) \frac{\log n}{n}\right] .
\end{aligned}
$$

By the Strong Law of Large Numbers (Appendix A, Theorem 26) we have that

$$
\begin{aligned}
& \sum_{\left(a, a_{\mathrm{ne}(v)}\right) \in A^{\mathrm{ne}(v)+1}} \frac{N\left(a, a_{\mathrm{ne}(v)}\right)}{n} \log \frac{\hat{p}\left(a \mid a_{\mathrm{ne}(v)}\right)}{\hat{p}\left(a \mid a_{W}\right)} \\
& \longrightarrow \sum_{\left(a, a_{\mathrm{ne}(v)}\right) \in A^{\mathrm{ne}(v)+1}} p\left(a, a_{\mathrm{ne}(v)}\right) \log \frac{p\left(a \mid a_{\mathrm{ne}(v)}\right)}{p\left(a \mid a_{W}\right)}
\end{aligned}
$$

almost surely as $n \rightarrow \infty$. On the other hand,

$$
c\left(|A|^{|\operatorname{ne}(v)|}-|A|^{|W|}\right) \frac{\log n}{n} \longrightarrow 0
$$

when $n \rightarrow \infty$. Now, note that we can rewrite the right-hand side of (5.7) by

$$
\begin{aligned}
\sum_{\left(a, a_{\mathrm{ne}(v)}\right) \in A^{\mathrm{ne}(v)+1}} p\left(a, a_{\mathrm{ne}(v)}\right) \log \frac{p\left(a \mid a_{\mathrm{ne}(v)}\right)}{p\left(a \mid a_{W}\right)} & \\
= & \sum_{a_{\mathrm{ne}(v)} \in A^{\mathrm{ne}(v)}} p\left(a_{\mathrm{ne}(v)}\right) \sum_{a \in A} p\left(a \mid a_{\mathrm{ne}(v)}\right) \log \frac{p\left(a \mid a_{\mathrm{ne}(v)}\right)}{p\left(a \mid a_{W}\right)} \\
= & \sum_{a_{\mathrm{ne}(v)} \in A^{\mathrm{ne}(v)}} p\left(a_{\mathrm{ne}(v)}\right) D\left(p\left(. \mid a_{\mathrm{ne}(v)}\right) ; p\left(. \mid a_{W}\right)\right) .
\end{aligned}
$$

By Lemma 23 (in Appendix A) and the minimality of ne $(v)$ (see (5.1) and Lemmas 10 and 11) we must have $D\left(p\left(. \mid a_{\mathrm{ne}(v)}\right) ; p\left(. \mid a_{W}\right)\right)>0$ for at least one $a_{\mathrm{ne}(v)}$, so

$$
\sum_{a_{\mathrm{ne}(v)} \in A^{\mathrm{ne}(v)}} p\left(a_{\mathrm{ne}(v)}\right) D\left(p\left(. \mid a_{\mathrm{ne}(v)}\right) ; p\left(. \mid a_{W}\right)\right)>0
$$

simultaneously for all $W \subset \operatorname{ne}(v)$, eventually almost surely as $n \rightarrow \infty$. Therefore

$$
\max _{W \in \mathcal{B}_{2}} \operatorname{PML}\left(x_{v}^{(1: n)} \mid x_{W}^{(1: n)}\right)<\operatorname{PML}\left(x_{v}^{(1: n)} \mid x_{\mathrm{ne}(v)}^{(1: n)}\right)
$$

eventually almost surely as $n \rightarrow \infty$, completing the proof of case (b).

(c) In this case, we have to prove that

$$
\max _{W \in \mathcal{B}_{3}} \operatorname{PML}\left(x_{v}^{(1: n)} \mid x_{W}^{(1: n)}\right)<\operatorname{PML}\left(x_{v}^{(1: n)} \mid x_{\mathrm{ne}(v)}^{(1: n)}\right)
$$

eventually almost surely as $n \rightarrow \infty$.

At first we will prove that

$$
\max _{W \in \mathcal{B}_{3}} \operatorname{PML}\left(x_{v}^{(1: n)} \mid x_{W}^{(1: n)}\right) \leq \operatorname{PML}\left(x_{v}^{(1: n)} \mid x_{V_{n} \backslash\{v\}}^{(1: n)}\right)
$$


eventually almost surely as $n \rightarrow \infty$. Note that we have

$$
\begin{aligned}
& \operatorname{PML}\left(x_{v}^{(1: n)} \mid x_{V_{n} \backslash\{v\}}^{(1: n)}\right)-\operatorname{PML}\left(x_{v}^{(1: n)} \mid x_{W}^{(1: n)}\right) \\
= & \sum_{\left(a, a_{V_{n} \backslash\{v\}}\right) \in A^{V_{n}}} N\left(a, a_{V_{n} \backslash\{v\}}\right) \log \frac{\hat{p}\left(a \mid a_{V_{n} \backslash\{v\}}\right)}{\hat{p}\left(a \mid a_{W}\right)}-c\left(|A|^{\left|V_{n}\right|-1}-|A|^{|W|}\right) \log n \\
= & n\left[\sum_{\left(a, a_{V_{n} \backslash\{v\}}\right) \in A^{V_{n}}} \frac{N\left(a, a_{V_{n} \backslash\{v\}}\right)}{n} \log \frac{\hat{p}\left(a \mid a_{V_{n} \backslash\{v\}}\right)}{\hat{p}\left(a \mid a_{W}\right)}-c\left(|A|^{\left|V_{n}\right|-1}-|A|^{|W|}\right) \frac{\log n}{n}\right] .
\end{aligned}
$$

The second term in the brackets

$$
c\left(|A|^{\left|V_{n}\right|-1}-|A|^{|W|}\right) \frac{\log n}{n} \longrightarrow 0
$$

when $n \rightarrow \infty$. And, for the first term we have

$$
\begin{aligned}
& \sum_{\left(a, a_{V_{n} \backslash\{v\}}\right) \in A^{V_{n}}} \frac{N\left(a, a_{V_{n} \backslash\{v\}}\right)}{n} \log \frac{\hat{p}\left(a \mid a_{V_{n} \backslash\{v\}}\right)}{\hat{p}\left(a \mid a_{W}\right)} \\
= & \sum_{\left(a, a_{V_{n} \backslash\{v\}}\right) \in A^{V_{n}}}\left[\frac{N\left(a, a_{V_{n} \backslash\{v\}}\right)}{n} \log \frac{\hat{p}\left(a \mid a_{V_{n} \backslash\{v\}}\right)}{\hat{p}\left(a \mid a_{W}\right)}-\frac{N\left(a, a_{V_{n} \backslash\{v\}}\right)}{n} \log p\left(a \mid a_{W}\right)\right. \\
+ & \left.\frac{N\left(a, a_{V_{n} \backslash\{v\}}\right)}{n} \log p\left(a \mid a_{W}\right)\right] \\
= & \sum_{\left(a, a_{V_{n} \backslash\{v\}}\right) \in A^{V_{n}}}\left[\frac{N\left(a, a_{V_{n} \backslash\{v\}}\right)}{n} \log \frac{\hat{p}\left(a \mid a_{V_{n} \backslash\{v\}}\right)}{p\left(a \mid a_{W}\right)}-\frac{N\left(a, a_{V_{n} \backslash\{v\}}\right)}{n} \log \frac{\hat{p}\left(a \mid a_{W}\right)}{p\left(a \mid a_{W}\right)}\right] .
\end{aligned}
$$

We divide again the expression in two parts. Loking at the second term of the sum in (5.8) we have

$$
\begin{aligned}
& \sum_{\left(a, a_{V_{n} \backslash\{v\}}\right) \in A^{V_{n}}} \frac{N\left(a, a_{V_{n} \backslash\{v\}}\right)}{n} \log \frac{\hat{p}\left(a \mid a_{W}\right)}{p\left(a \mid a_{W}\right)} \\
= & \sum_{\left(a, a_{W}\right) \in A^{W+1}} \log \frac{\hat{p}\left(a \mid a_{W}\right)}{p\left(a \mid a_{W}\right)} \sum_{a_{V_{n} \backslash v \cup W} \in A^{V_{n} \backslash v \cup W}} \frac{N\left(a, a_{V_{n} \backslash\{v\}}\right)}{n} \\
= & \sum_{\left(a, a_{W}\right) \in A^{W+1}} \frac{N\left(a, a_{W}\right)}{n} \log \frac{\hat{p}\left(a \mid a_{W}\right)}{p\left(a \mid a_{W}\right)} \\
= & \sum_{\left(a, a_{W}\right) \in A^{W+1}} \frac{N\left(a_{W}\right)}{n} \hat{p}\left(a \mid a_{W}\right) \log \frac{\hat{p}\left(a \mid a_{W}\right)}{p\left(a \mid a_{W}\right)} \\
= & \sum_{\left(a, a_{W}\right) \in A^{W+1}} \frac{N\left(a_{W}\right)}{n} D\left(\hat{p}\left(\cdot \mid a_{W}\right) ; p\left(\cdot \mid a_{W}\right)\right) .
\end{aligned}
$$

By Lemma 24 (in Appendix A) and Corollary 21, we have that eventually almost surely

$$
\begin{aligned}
& \max _{W \in \mathcal{B}_{3}} \sum_{\left(a, a_{W}\right) \in A^{W+1}} \frac{N\left(a_{W}\right)}{n} D\left(\hat{p}\left(\cdot \mid a_{W}\right) ; p\left(\cdot \mid a_{W}\right)\right) \\
\leq & \max _{W \in \mathcal{B}_{3}} \frac{16|A|^{|W|+1} \log n}{n p_{*}^{|W|+2}} \leq \frac{16|A|^{\left|V_{n}\right|} \log n}{n p_{*}^{\left|V_{n}\right|+1}} \longrightarrow 0
\end{aligned}
$$

as $n \rightarrow \infty$. 
Now, as $\hat{p}\left(a \mid a_{V_{n} \backslash\{v\}}\right)$ is the maximum likelihood estimator of $p\left(a \mid a_{V_{n} \backslash\{v\}}\right)$ and $V_{n}$ will eventually contain ne $(v)$, the first term in the sum (5.8) can be lower-bounded by

$$
\begin{aligned}
& \sum_{\left(a, a_{V_{n} \backslash\{v\}}\right) \in A^{V_{n}}} \frac{N\left(a, a_{V_{n} \backslash\{v\}}\right)}{n} \log \frac{p\left(a \mid a_{V_{n} \backslash\{v\}}\right)}{p\left(a \mid a_{W}\right)} \\
= & \sum_{\left(a, a_{V_{n} \backslash\{v\}}\right) \in A^{V_{n}}} \frac{N\left(a, a_{V_{n} \backslash\{v\}}\right)}{n} \log \frac{p\left(a \mid a_{\mathrm{ne}(v)}\right)}{p\left(a \mid a_{W}\right)} \\
= & \sum_{\left(a, a_{V_{n} \backslash\{v\}}\right) \in A^{V_{n}}} \hat{p}\left(a, a_{V_{n} \backslash\{v\}}\right) \log \frac{p\left(a \mid a_{\mathrm{ne}(v)}\right)}{p\left(a \mid a_{W}\right)}
\end{aligned}
$$

By Theorem 20, $\hat{p}\left(a, a_{V_{n} \backslash\{v\}}\right)>p\left(a, a_{V_{n} \backslash\{v\}}\right)-\sqrt{\frac{\delta \log n}{n}}$. Ergo, for (5.10) we have

$$
\begin{aligned}
& \sum_{\left(a, a_{V_{n} \backslash\{v\}}\right) \in A^{V_{n}}} \hat{p}\left(a, a_{V_{n} \backslash\{v\}}\right) \log \frac{p\left(a \mid a_{\mathrm{ne}(v)}\right)}{p\left(a \mid a_{W}\right)} \\
> & \sum_{\left(a, a_{V_{n} \backslash\{v\}}\right) \in A^{V_{n}}}\left[p\left(a, a_{V_{n} \backslash\{v\}}\right)-\sqrt{\frac{\delta \log n}{n}}\right] \log \frac{p\left(a \mid a_{\mathrm{ne}(v)}\right)}{p\left(a \mid a_{W}\right)} \\
= & \sum_{\left(a, a_{V_{n} \backslash\{v\}}\right) \in A^{V_{n}}} p\left(a_{V_{n} \backslash\{v\}}\right) p\left(a \mid a_{V_{n} \backslash\{v\}}\right) \log \frac{p\left(a \mid a_{\mathrm{ne}(v)}\right)}{p\left(a \mid a_{W}\right)} \\
- & \sum_{\left(a, a_{V_{n} \backslash\{v\}}\right) \in A^{V_{n}}} \sqrt{\frac{\delta \log n}{n}} \log \frac{p\left(a \mid a_{\mathrm{ne}(v)}\right)}{p\left(a \mid a_{W}\right)} \\
\geq & \sum_{\left(a_{V_{n} \backslash\{v\}}\right) \in A^{V_{n}}-1} p\left(a_{V_{n} \backslash\{v\}}\right) \sum_{a \in A} p\left(a \mid a_{\mathrm{ne}(v)}\right) \log \frac{p\left(a \mid a_{\mathrm{ne}(v)}\right)}{p\left(a \mid a_{W}\right)} \\
= & \sum_{\left(a, a_{V_{n} \backslash\{v\}}\right) \in A^{V_{n}}} \sqrt{\frac{\delta \log n}{n}} \log \frac{1}{p_{\min }(v)} \\
+ & \sum_{\left(a_{V_{n} \backslash\{v\}}\right) \in A^{V_{n}}-1} p\left(a_{V_{n} \backslash\{v\}} D\left(p\left(\cdot \mid a_{\mathrm{ne}(v)}\right) ; p\left(\cdot \mid a_{W}\right)\right)\right. \\
+ & \log p_{\min }(v) \sqrt{\frac{\delta \log n}{n}}
\end{aligned}
$$

By Assumptions 14 and 17, (5.11) is grater than

$$
\alpha+|A|^{\left|V_{n}\right|} \log p_{\min (v)} \sqrt{\frac{\delta \log n}{n}} \geq \frac{\alpha}{2}>0
$$

eventually almost surely as $n \rightarrow \infty$, simultaneously for all $W \in \mathcal{B}_{3}$. So,

$$
\max _{W \in \mathcal{B}_{3}} \operatorname{PML}\left(x_{v}^{(1: n)} \mid x_{W}^{(1: n)}\right) \leq \operatorname{PML}\left(x_{v}^{(1: n)} \mid x_{V_{n} \backslash\{v\}}^{(1: n)}\right)
$$

eventually almost surely as $n \rightarrow \infty$.

As $V_{n}$ will contain ne $(v)$ eventually as $n \rightarrow \infty$, then $V_{n} \backslash\{v\} \in \mathcal{B}_{1}$, eventually as $n \rightarrow \infty$. Then, by item (a),

$$
\operatorname{PML}\left(x_{v}^{(1: n)} \mid x_{V_{n} \backslash\{v\}}^{(1: n)}\right) \leq \max _{W \in \mathcal{B}_{1}} \operatorname{PML}\left(x_{v}^{(1: n)} \mid x_{W}^{(1: n)}\right)<\operatorname{PML}\left(x_{v}^{(1: n)} \mid x_{\operatorname{ne}(v)}^{(1: n)}\right) .
$$


So,

$$
\max _{W \in \mathcal{B}_{3}} \operatorname{PML}\left(x_{v}^{(1: n)} \mid x_{W}^{(1: n)}\right)<\operatorname{PML}\left(x_{v}^{(1: n)} \mid x_{\mathrm{ne}(v)}^{(1: n)}\right)
$$

eventually almost surely as $n \rightarrow \infty$. This finishes the proof of case (c).

By combining the results of all three cases, we conclude that

$$
\max _{W \subset V_{n} \backslash\{v\}, W \neq \mathrm{ne}(v)} \operatorname{PML}\left(x_{v}^{(1: n)} \mid x_{W}^{(1: n)}\right)<\operatorname{PML}\left(x_{v}^{(1: n)} \mid x_{\mathrm{ne}(v)}^{(1: n)}\right)
$$

and $\widehat{\mathrm{ne}}(v)=\operatorname{ne}(v)$, eventually almost surely as $n \rightarrow \infty$.

Proof of Corollary 19. The proof of the corollary follows from Theorem 18 and noting that $V^{\prime}$ is finite. The same arguments of the proof of Corollary 8 can be used here just changing the finite $V$ by $V^{\prime}$, which is also finite. 


\section{Chapter 6}

\section{Conclusion}

In this thesis we introduced a new estimator for discrete Markov random fields on graphs, and we proved its consistency even for the case where the set of vertices is countable infinite (Chapter 5). In this case we highlight the theoretical difficulties and the differences between this and the case where the set of vertices is finite (Chapter 2). Some propositions had to be changed or even exchanged for other more powerful results. And even for the case where the set of vertices is countable infinite, we did not need to assume that the model satisfies the usual "positivity" condition.

To estimate the graph estructure, i.e. to estimate the set of edges $E$, we first estimate for each vertex its neighborhood, based on a penalized maximum condicional likelihood criterion. We showed that this estimator converges almost surely to the true set of neighbors. Then we showed that, combing the estimators of all vertices, the estimator of $G=(V, E)$ converges almost surely to the true graph. When we are dealing with a countable infinite set of vertices we only estimate a finite subgraph of $G$, in this case the estimator converges almost surely to the true finite subgraph.

The results obtained by the simulations in Chapter 3 corroborated the theoretical statements about the consistency of the estimator. We were able to evaluate the performance of the estimator from different angles: considering the sample size, the value of the constant $c$ of the estimator, and the choice between a conservative or non conservative approach. Through these evaluations we were able to conclude on the superiority of the non conservative approach. Also we were able to see the impact caused by the value of $c$, transforming the estimator in more or less conservative. The higher the value of $c$, the more conservative is the estimator. On the other hand, for fixed $c$, we observed the convergence of the estimated graphs when the sample size grows, confirming the consistency of the estimator. Other factors related to the complexity of the model influence the estimation, as the number of vertices, the alphabet size and the dependence structure of the model (represented by the graph). Few vertices, small alphabets, and graphs with few edges are easier to estimate, in the sense that require smaller samples and are computationally less expensive.

We can apply this model to several real data, especially when we are interested in discovering the conditional dependence structure of the variables involved. Of course, some considerations must be made, our model deals with discrete variables on finite alphabets, and to estimate the models the samples must have independent observations. When in real data the variables are continuous we can discretized them, and this discretization should make sense for the application. But a more difficult problem to handle with is the lack of independence between the sample observations. To overcome this issue some methods can be used, as for example reduce the sample taking only observations separated by a gap. This gap can be choosen based on the autocorrelation function, as in the case of EEG signals presented in this thesis.

In many cases of real data applications we have a lot of variables (represented in the graph by its vertices) and sample sizes that are not large enough. In these cases all information about the absence of edges should be used to simplify the problem. Because we estimate the set of neighbors of each vertex, this information can be used in a more simple way. And the program used here to estimate the model has the option of inciating the algoritm with an adjacency matrix different from the complete graph. 
In the applications we introduced an empirical method to discover the best value of the constant $c$, based on $k$-fold cross validation, although other methods are also possible, as for example the bootstrap. Though, the question of how to choose the best constant $c$ remains open, and we intend to continue to work on it in the future. 


\section{Appendix A}

\section{Basic probability results}

Definition 22. (Küllback-Leibler divergence) The Küllback-Leibler divergence between the two probabilities distribution $P$ and $Q$ over $A$ is defined by

$$
D(P ; Q)=\sum_{a \in A} P(a) \log \frac{P(a)}{Q(a)}
$$

where, by convention, $P(a) \log \frac{P(a)}{Q(a)}=0$ if $P(a)=0$ and $P(a) \log \frac{P(a)}{Q(a)}=+\infty$ if $P(a)>Q(a)=0$.

Lemma 23. For the Küllback-Leibler divergence, we have that $D(P ; Q) \geq 0$ and $D(P ; Q)=0$ if and only if $P(a)=Q(a) \forall a \in A$.

The following lemma was taken from Csiszár and Talata (2006, Lemma 6.3).

Lemma 24. For any $P$ and $Q$ we have

$$
D(P ; Q) \leq \sum_{a \in A: Q(a)>0} \frac{[P(a)-Q(a)]^{2}}{Q(a)} .
$$

Definition 25. (Empirical mean) Let $X_{1}, X_{2}, \ldots, X_{n}$ be random variables, the empirical mean $(\bar{X})$ of this variables is defined by

$$
\bar{X}=\frac{1}{n} \sum_{i=1}^{n} X_{i} .
$$

This next theorem can be found in Breiman (1968, Section 3.9).

Theorem 26. (Law of the Iterated Logarithm) Let $Y_{1}, Y_{2}, \ldots$ be independent identically distributed random variables with $\mathbb{E}\left[Y_{i}\right]=0$ and $\mathbb{E}\left[Y_{i}^{2}\right]<\infty$, and $Z_{n}=\sum_{i=1}^{n} Y_{i}$ then

$$
\limsup _{n \rightarrow \infty} \frac{\left|Z_{n}\right|}{\mathbb{E}\left[Y_{i}^{2}\right] \sqrt{2 n \log \log n}}=1 \text { almost surelly. }
$$

And this theorem can be found in Breiman (1968, Section 3.6).

Theorem 27. (Strong Law fo Large Numbers) Let $X_{1}, X_{2}, \ldots$ be independent identically distributed random variables with $\mathbb{E}\left[X_{i}\right]=\mu$ and $\mathbb{E}\left[\left|X_{i}\right|\right]<\infty$, then

$$
\bar{X} \rightarrow \mu \text { almost surelly as } n \rightarrow \infty \text {. }
$$

The next theorem and its proof can be found in Hoeffding (1963). 
Theorem 28. (Hoeffding's inequality) Let $X_{1}, \ldots, X_{n}$ be independent random variables where $X_{i}$ are strictly bounded by the intervals $\left[a_{i}, b_{i}\right]$. Then, for all $t>0$

$$
\mathbb{P}(|\bar{X}-\mathbb{E}[\bar{X}]| \geq t) \leq \exp \left(-\frac{2 n^{2} t^{2}}{\sum_{i=1}^{n}\left(b_{i}-a_{i}\right)^{2}}\right) .
$$




\section{Appendix B}

\section{R Programs}

\section{B.1 Generating samples}

Here are presented the $\mathrm{R}$ programs that genarete samples from the examples in Chapter 2, whose algorithms are described in Section 3.1 of Chapter 3. The following programs can generate samples of size $n$ for each Markov random field given as examples. Remember that because the theoretical probabilities described in Chapter 2 are fixed, they are not parameters of the created function.

\section{B.1.1 Example 1}

The following program generates a sample of size $n$ of the Markov random field in Example 1 (Section 3.1), with 5 variables in the aphabet $A=(0,1,2)$.

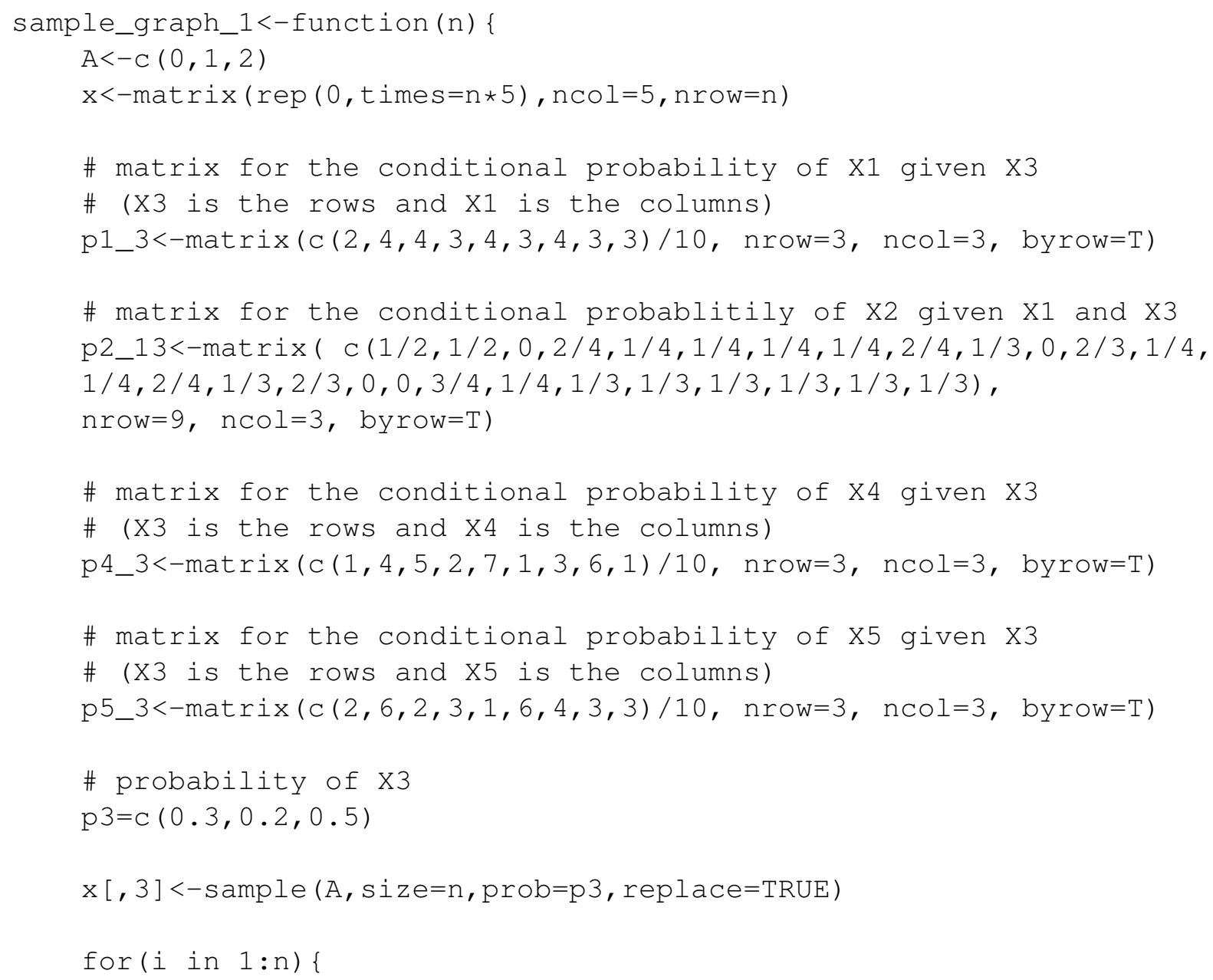




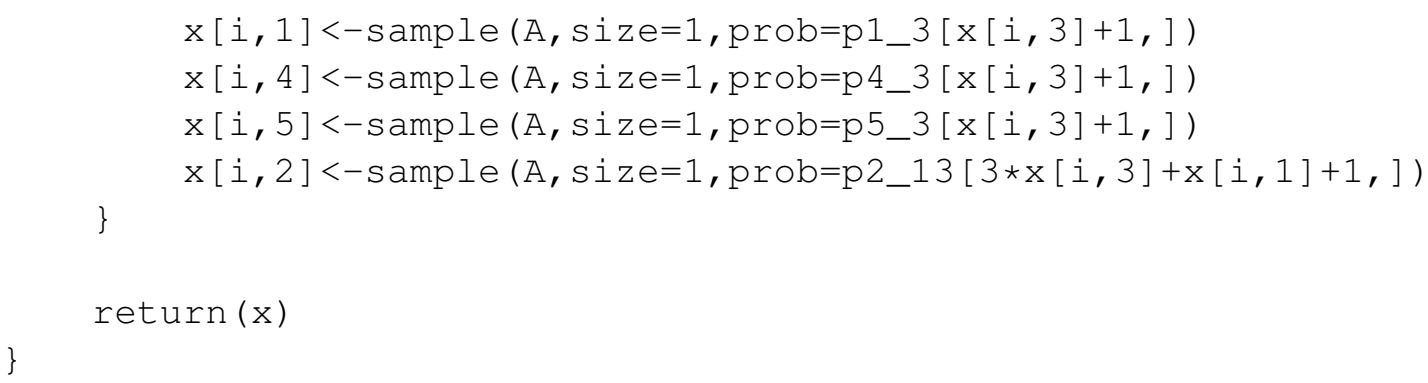

\section{B.1.2 Example 2}

The following program generates a sample of size $n$ of the Markov random field in Example 2 (Section 3.1), with 7 variables in the aphabet $A=(0,1)$.

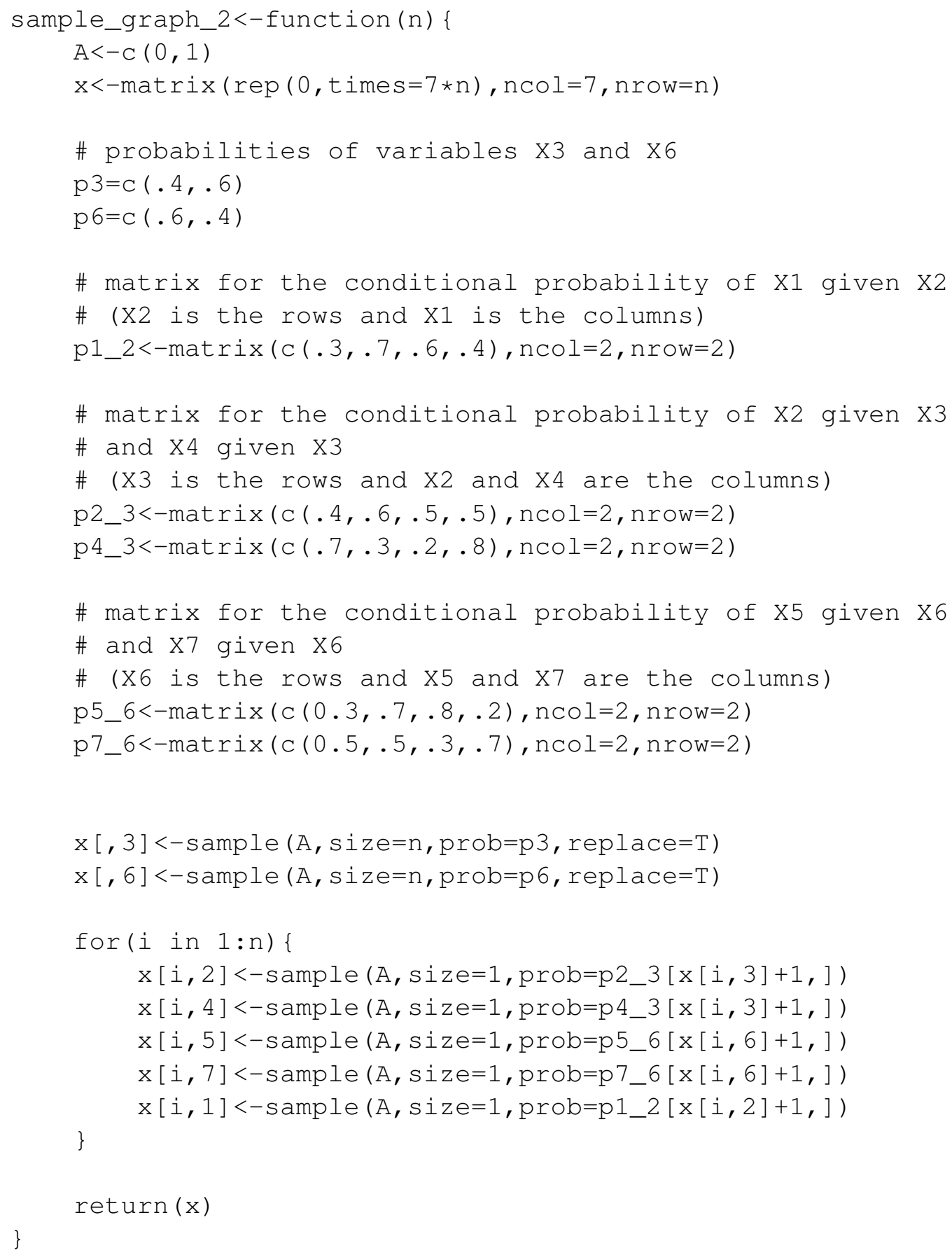




\section{B.1.3 Example 3}

The following program generates a sample of size $n$ of the Markov random field in Example 3 (Section 3.1), with 12 variables in the aphabet $A=(0,1,2)$.

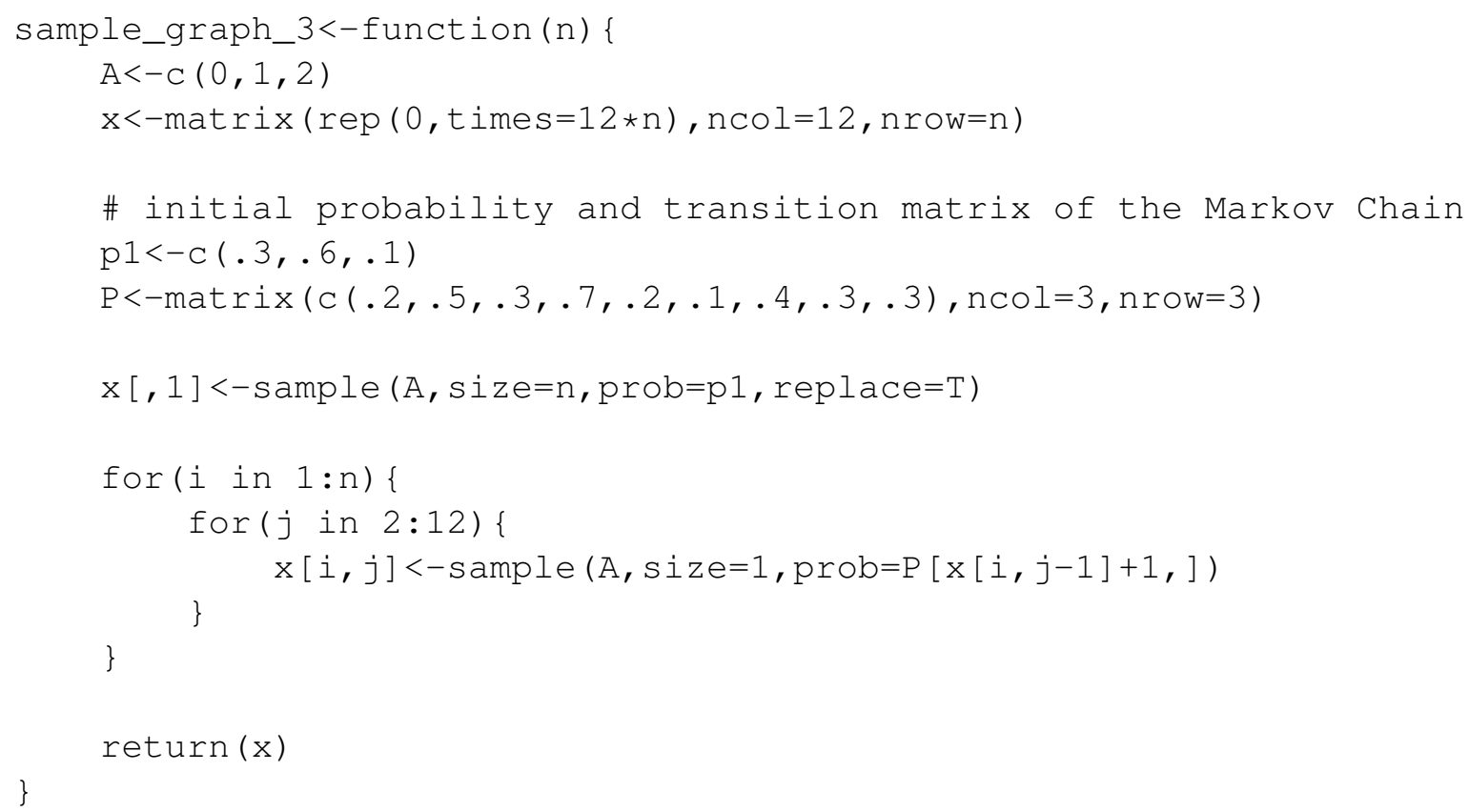

\section{B.1.4 Example 4}

The following program generates a sample of size $n$ of the Markov random field in Example 4 (Section 3.1), with 10 variables in the aphabet $A=(0,1,2)$.

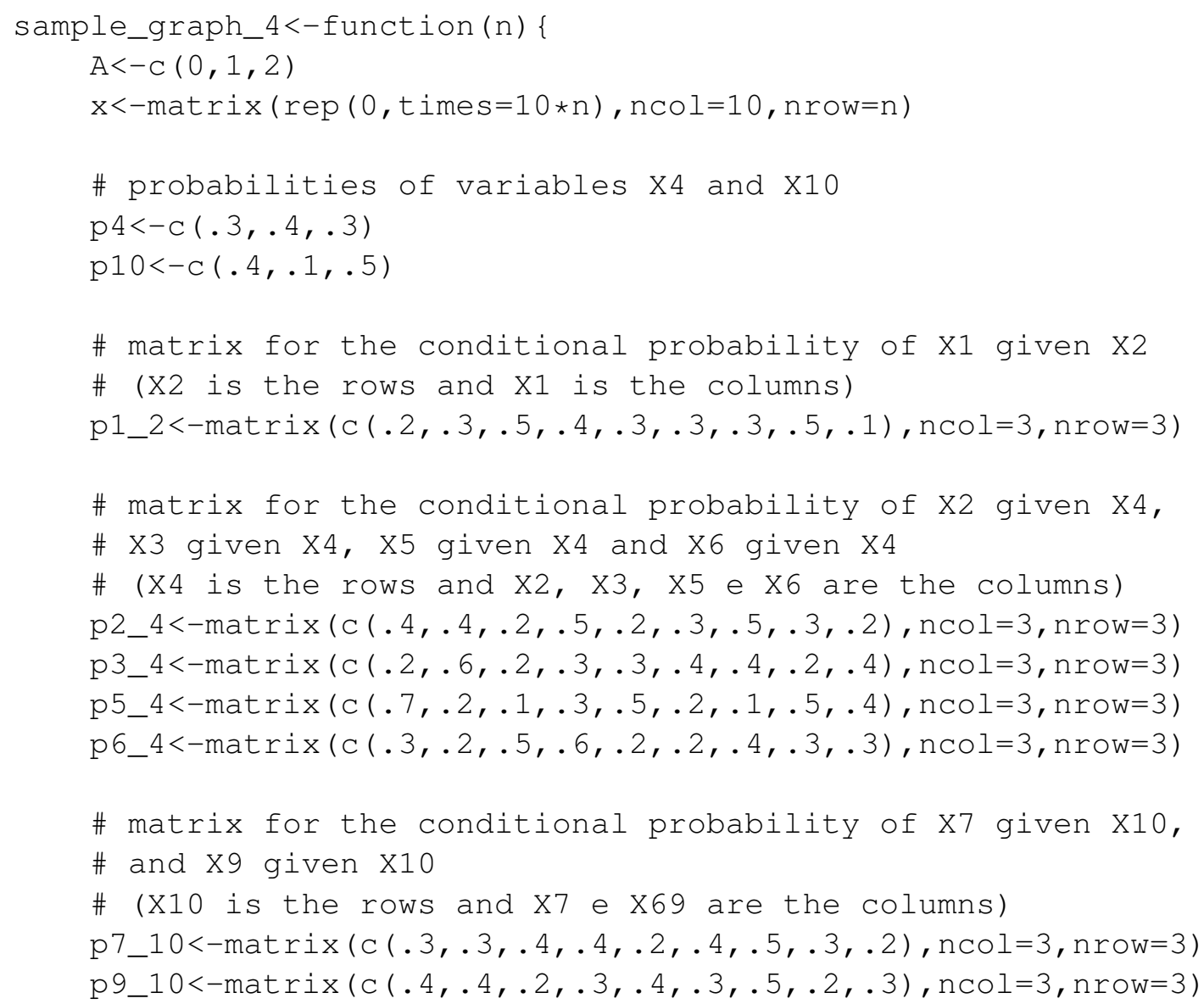




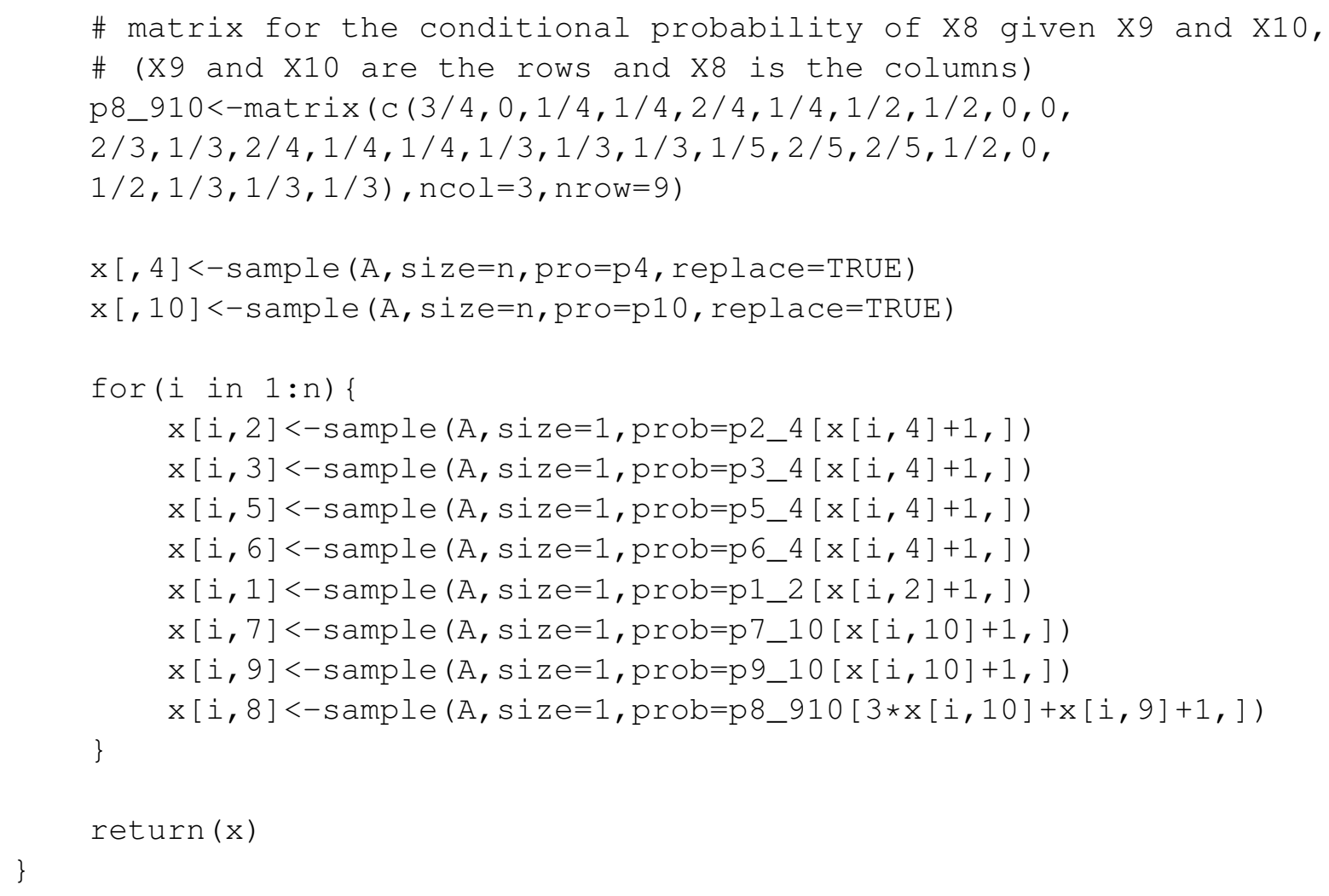

\section{B.2 Programs that measure the estimator performance}

Here are presented the $\mathrm{R}$ programs that were used to measure the estimator performance in Chapter 3. Because the adjacency matrix is simetric, it is possible to represent those matrices using vectors in such a way that a $p \times p$ matrix is represented by a vector of length $0.5 p(p-1)$. This next example tries to explain how this tranformation works, remember that in the adjacency matrix all the numbers on the diagonal are equal to zero and that it is symetric, then:

$$
\left[\begin{array}{lllll}
0 & a & b & c & d \\
a & 0 & e & f & g \\
b & e & 0 & h & i \\
c & f & h & 0 & j \\
d & g & i & j & 0
\end{array}\right] \Rightarrow\left[\begin{array}{llllllllll}
a & b & c & d & e & f & g & h & i & j
\end{array}\right]
$$

So this next $\mathrm{R}$ program is a function that transforms an adjacency matrix into a vector:

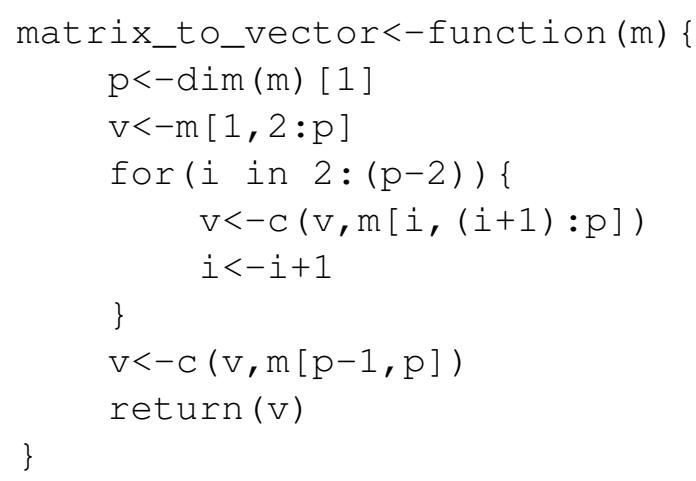

The next $\mathrm{R}$ program calculates the underestimation, overestimation and total errors given the true graph and an estimative for this graph. Both have to be represented by vectors, not by their adjacency matrices. The parameter $g$ is the vector that represents the real graph, and eg is the vector 
that represents the estimated graph. The function returns the underestimation, the overestimation and the total errors, in this order.

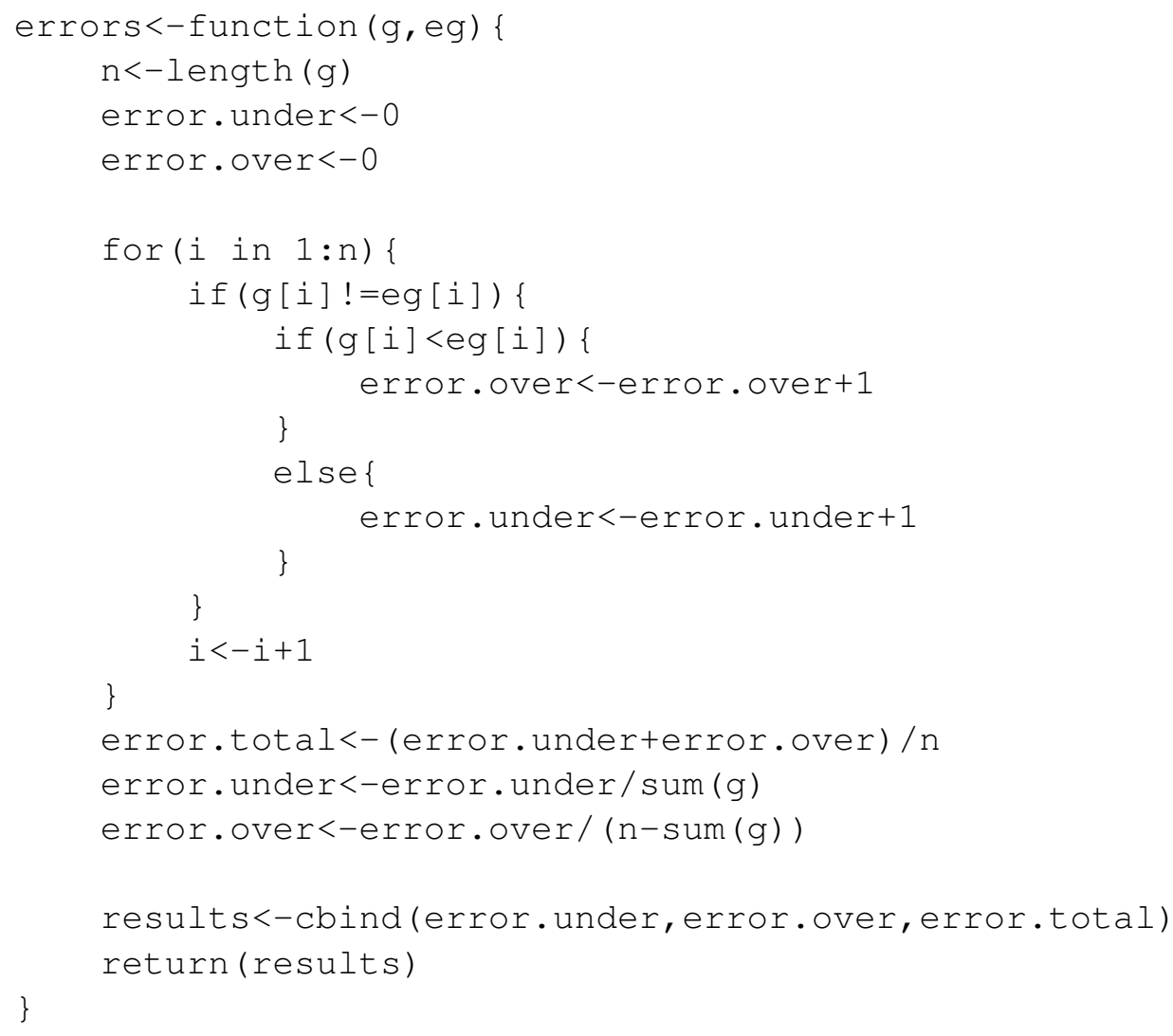

Now, this last $\mathrm{R}$ program is a function that gives the pair (false positive rate, true positive rate $)=(1-$ specificity, sensibility $)$ of the ROC curve. Again the vectores are used instead of the adjacency matrices. And the parameter $g$ represents the real graph vector and eg represents the estimated graph vector.

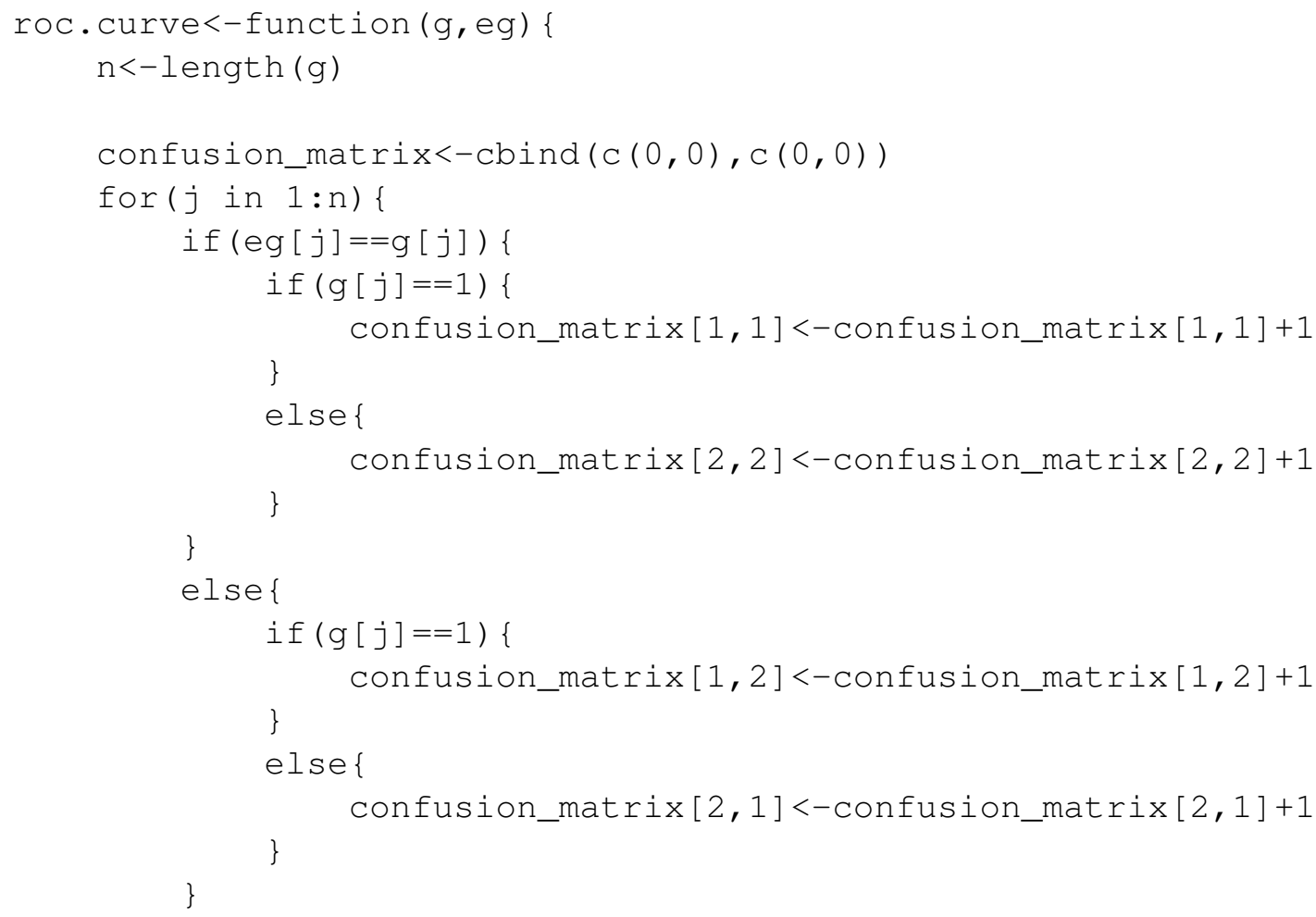




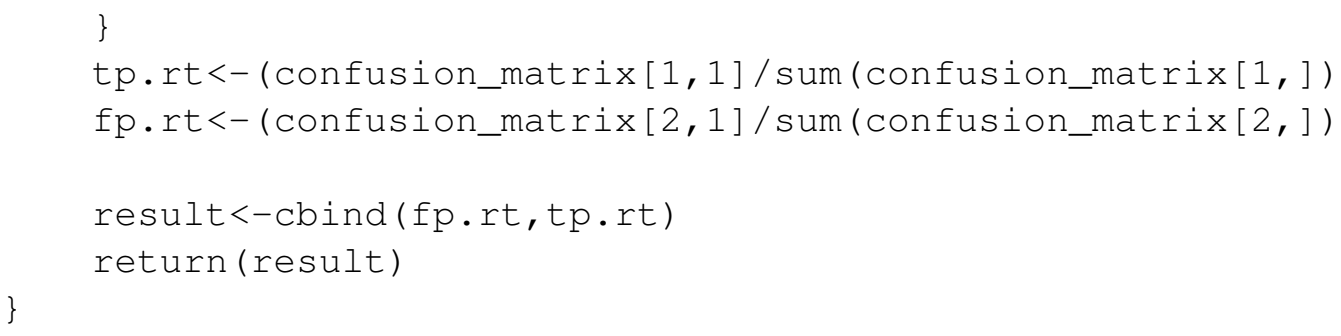

\section{B.3 Log likelihood for cross-validation}

The following $\mathrm{R}$ program is a function that calculates the log likelihood needed in the crossvalidation method (Chapter 4). Its parameters are: m.adj that represents the adjacency matrix of neighbors estimated using the training sample, s.training the training sample, and s.test the test sample.

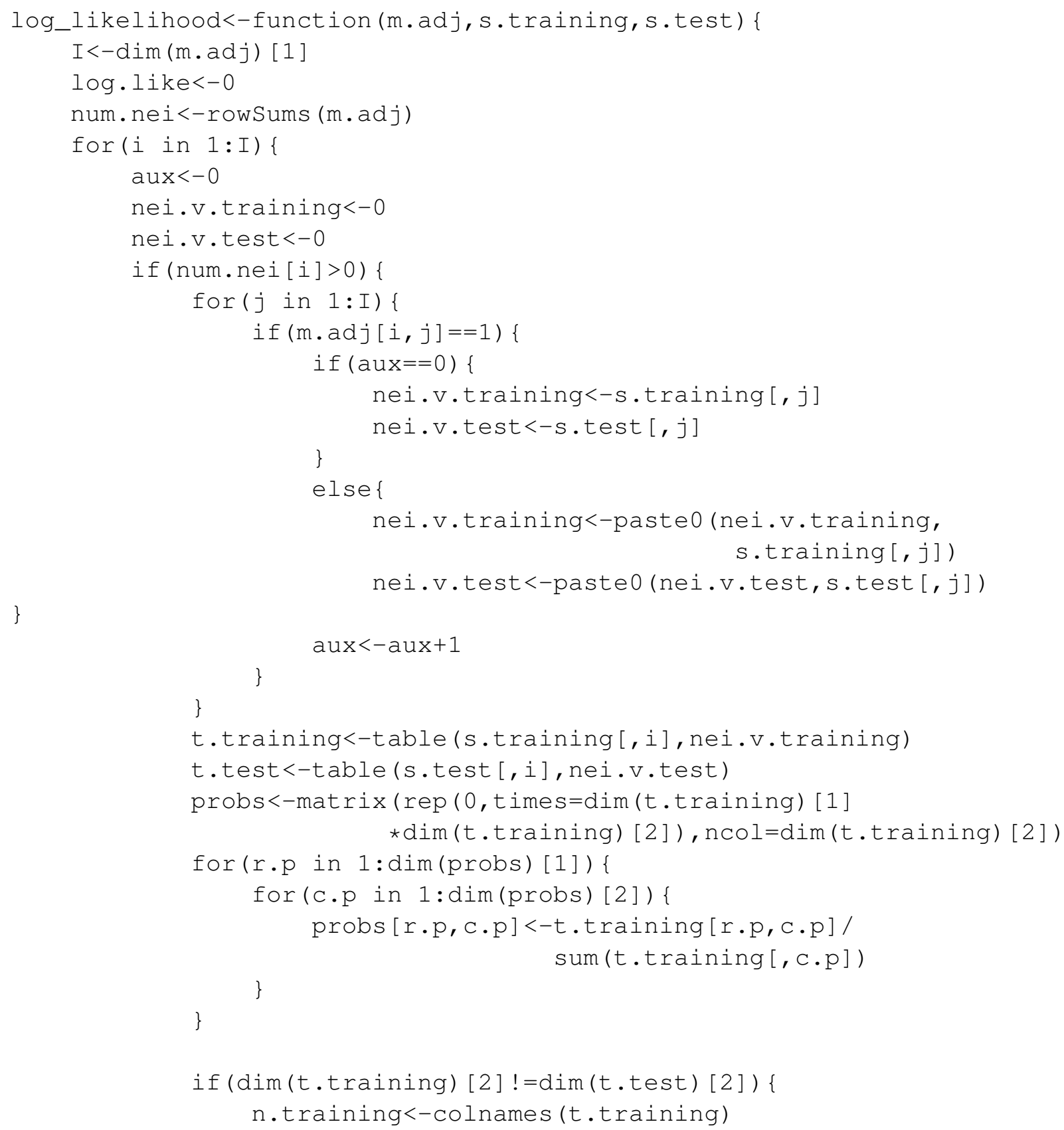




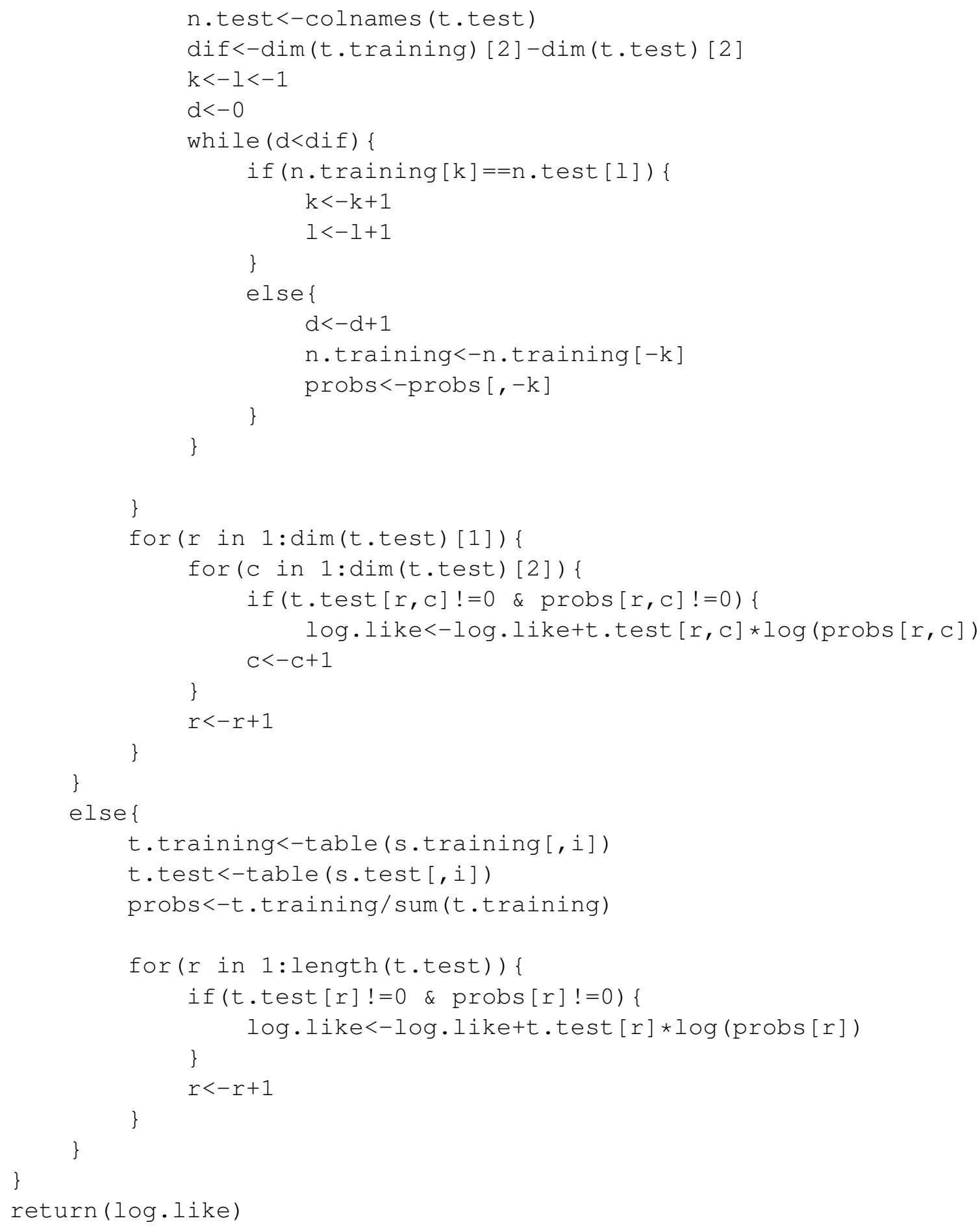




\section{Bibliography}

Leo Breiman. Probability. Addison-Wesley, Reading, Massachusets, first edition, 1968. 53

P. Collinson. Of bombers, radiologists, and cardiologists: time to ROC. Heart, 80(3):215-217, 1998. 31

Imre Csiszár. Large-scale typicality of Markov sample paths and consistency of MDL order estimators. IEEE Trans. Inform. Theory, 48(6):1616-1628, 2002. ISSN 0018-9448. doi: 10.1109/TIT.2002.1003842. URL http://dx.doi.org/10.1109/TIT.2002.1003842. Special issue on Shannon theory: perspective, trends, and applications. 43

Imre Csiszár and Zsolt Talata. Consistent estimation of the basic neighborhood of Markov random fields. Ann. Statist., 34(1):123-145, 2006. ISSN 0090-5364. doi: 10.1214/009053605000000912. URL http://dx.doi.org/10.1214/009053605000000912. 1, 4, 41, 53

Tom Fawcett. An Introduction to ROC Analysis. Pattern Recogn. Lett., 27(8):861-874, jun 2006. ISSN 0167-8655. doi: 10.1016/j.patrec.2005.10.010. URL http://dx.doi.org/10.1016/j.patrec. 2005.10.010. 22

Antonio Galves, Enza Orlandi, and Daniel Y. Takahashi. Identifying interacting pairs of sites in Ising models on a countable set. Braz. J. Probab. Stat., 29(2):443-459, 2015. 1

Hans-Otto Georgii. Gibbs measures and phase transitions, volume 9 of de Gruyter Studies in Mathematics. Walter de Gruyter \& Co., Berlin, second edition, 2011.

Trevor Hastie, Robert Tibshirani, and Jerome Friedman. The elements of statistical learning: data mining, inference and prediction. Springer, 2 edition, 2009. URL http://www-stat.stanford.edu/ $\sim$ tibs/ElemStatLearn/. 13, 35

Wassily Hoeffding. Probability inequalities for sums of bounded random variables. Journal of the American Statistical Association, 58(301):pp. 13-30, 1963. ISSN 01621459. URL http://www. jstor.org/stable/2282952. 53

Gareth James, Daniela Witten, Trevor Hastie, and Robert Tibshirani. An Introduction to Statistical Learning: With Applications in R. Springer Publishing Company, Incorporated, 2014. ISBN 1461471370, 9781461471370. 35

Daphne Koller and Nir Friedman. Probabilistic Graphical Models: Principles and Techniques - Adaptive Computation and Machine Learning. The MIT Press, 2009. ISBN 0262013193, 9780262013192. 1

Steffen L. Lauritzen. Graphical Models. Oxford University Press, 1996. 1, 4

Po-Ling Loh and Martin J. Wainwright. Structure estimation for discrete graphical models: Generalized covariance matrices and their inverses. Ann. Statist., 41(6):3022-3049, 2013. 1

N. Meinshausen and P. Bühlmann. High-dimensional graphs and variable selection with the lasso. Ann. Statist., 34(3):1436-1462, 2006. 1 
Pradeep Ravikumar, Martin J. Wainwright, and John D. Lafferty. High-dimensional Ising model selection using $l_{1}$-regularized logistic regression. Ann. Statist., 38(3):3022-1319, 2010. 1

Benjamin Reiser and David Faraggi. Confidence Intervals for the Generalized ROC Criterion. Biometrics, 53(2):644-652, 1997. ISSN 0006341X, 15410420. URL http://www.jstor.org/stable/ 2533964. 31

Maria L. Rizzo. Statistical Computing whit R, volume 9 of Computer Science and Data Analysis Series. Chapman \& Hall/CRC, first edition, 2007. 20

G. Schwarz. Estimating the dimension of a model. Ann. Statist., 6:461-464, 1978. 1, 3, 13

A. Shojaie and G. Michailidis. Penalized likelihood methods for estimation of sparse highdimensional directed acyclic graphs. Biometrika, 97(3):519-538, 2010. 1

David Strauss and Michael Ikeda. Pseudolikelihood Estimation for Social Networks. Journal of the American Statistical Association,, 85(409):204-212, 1990. 1

R. Tibshirani. Regression shrinkage and selection via the lasso. J. Roy. Statist. Soc. Ser. B, 58(1): 267-288, 1996. 1

C. S. Wallace and D. M. Boulton. An information measure for classification. Computer Journal, 11(2):185-194, 1968. 13

M. H. Zweig and G Campbell. Receiver-operating characteristic (ROC) plots: a fundamental evaluation toll in clinical medicine. Clinical chemistry, 39(4):561-577, 1993. 30 